Florida International University FIU Digital Commons

$11-20-2012$

\title{
Test of a Smock System on CPR Primary Emergency Measures and Medical Errors During Simulated Emergencies
}

Ruth Thomas

Florida International University, rthom005@fiu.edu

DOI: $10.25148 /$ etd.FI12113011

Follow this and additional works at: https://digitalcommons.fiu.edu/etd

\section{Recommended Citation}

Thomas, Ruth, "Test of a Smock System on CPR Primary Emergency Measures and Medical Errors During Simulated Emergencies" (2012). FIU Electronic Theses and Dissertations. 759.

https://digitalcommons.fiu.edu/etd/759 


\section{FLORIDA INTERNATIONAL UNIVERSITY \\ Miami, Florida}

TEST OF A SMOCK SYSTEM ON CPR PRIMARY EMERGENCY MEASURES AND MEDICAL ERRORS DURING SIMULATED EMERGENCIES

A dissertation submitted in partial fulfillment of

the requirements for the degree of

DOCTOR OF PHILOSOPHY

in

NURSING

by

Ruth Thomas

2012 
To: $\quad$ Dean Ora Lea Strickland

College of Nursing and Health Sciences

This dissertation, written by Ruth Thomas, and entitled Test of a Smock System on CPR Primary Emergency Measures and Medical Errors during Simulated Emergencies, having been approved in respect to style and intellectual content, is referred to you for judgment.

We have read this dissertation and recommend that it be approved.

$\begin{array}{r}\text { Mary Jo Trepka } \\ \hline \text { JoAnne Youngblut } \\ \hline \text { Jeffrey Groom } \\ \hline \text { othy Brooten, Major Professor }\end{array}$

Date of Defense: November 20, 2012

The dissertation of Ruth Thomas is approved.

Dean Ora Lea Strickland College of Nursing and Health Sciences

Dean Lakshmi N. Reddi University Graduate School

Florida International University, 2012 


\section{DEDICATION}

I dedicate this dissertation to my parents. With their limited education due to many insurmountable adversities they have achieved their impossible dream though me. Also, I

dedicate this dissertation to my husband who provided unwavering faith and continuous support throughout this very long journey. 


\section{ACKNOWLEDGMENTS}

The writing of this dissertation has been one of the most significant academic achievements in my career although there were many personal challenges. First I would like to thank God for giving me the desire to strive for the highest education and to believe that nothing is impossible. I thank my esteemed Professors, Drs. Dorothy Brooten, JoAnne Youngblut, Jeffrey Groom, and Mary Jo Trepka for their wisdom, knowledge, and commitment to high standards and expert guidance. This dissertation would not have been possible without their expert guidance.

A special appreciation goes to my parents Bishop Willie R. Everett and Louisa Everett who gave me tremendous encouragement throughout my life and stressed the importance of education. I thank you for your continuous prayers, understanding, and lifelong encouragement of my academic career. A special thanks to my husband Willie J. Thomas I love you dearly; my daughter and best friend Shamia Curry, you have taught me so much about sacrifice and compromise; my son Jeremy who keeps me grounded and prayerful; my son in-law Nathaniel Curry who works diligently; and my two beautiful granddaughters, Nyla and Nia who was born during the journey. Also, a special thanks to my family members (brothers, sisters, in-laws, cousins, nieces, nephews, aunts, and uncles) for their continued words of encouragement.

A special thanks to my co-workers at the University of Miami-Jackson Memorial Hospital who helped with the project and encouraged me daily. My bosses Drs. David J. Birnbach and Joshua Lenchus who believed in the patient safety project and allowed me to use the facilities. I would like to thank all of the nurses who participated in the study and all of the nurse educators who fully supported the study. Thanks to Dr. Kim and the use of the Ottawa abbreviated CRM checklist. 


\section{ABSTRACT OF THE DISSERTATION}

\section{TEST OF A SMOCK SYSTEM ON CPR PRIMARY EMERGENCY MEASURES AND MEDICAL ERRORS DURING SIMULATED EMERGENCIES}

by

\section{Ruth Thomas}

Florida International University, 2012

Miami, Florida

Dorothy Brooten, Major Professor

Rates of survival of victims of sudden cardiac arrest (SCA) using cardio pulmonary resuscitation (CPR) have shown little improvement over the past three decades. Since registered nurses (RNs) comprise the largest group of healthcare providers in U.S. hospitals, it is essential that they are competent in performing the four primary measures (compression, ventilation, medication administration, and defibrillation) of CPR in order to improve survival rates of SCA patients. The purpose of this experimental study was to test a color-coded SMOCK system on:1) time to implement emergency patient care measures 2) technical skills performance 3) number of medical errors, and 4) team performance during simulated CPR exercises. The study sample was $260 \mathrm{RNs}(\mathrm{M} 40$ years, $\mathrm{SD}=11.6)$ with work experience as an $\mathrm{RN}(\mathrm{M} 7.25$ years, $\mathrm{SD}=9.42$ ).Nurses were allocated to a control or intervention arm consisting of 20 groups of 5-8 RNs per arm for a total of 130 RNs in each arm. Nurses in each study arm were given clinical scenarios requiring emergency CPR. Nurses in the intervention group wore different color labeled aprons (smocks) indicating their role assignment (medications, ventilation, compression, defibrillation, etc) on the code team during CPR. Findings indicated that the intervention using color-labeled smocks for pre-assigned roles had a significant effect on the time nurses started 
compressions ( $\mathrm{t}=3.03, \mathrm{p}=0.005)$, ventilations $(\mathrm{t}=2.86, \mathrm{p}=0.004)$ and defibrillations $(\mathrm{t}=2.00$, $\mathrm{p}=.05$ ) when compared to the controls using the standard of care. In performing technical skills, nurses in the intervention groups performed compressions and ventilations significantly better than those in the control groups. The control groups made significantly $(t=-2.61, p=0.013)$ more total errors (7.55 SD 1.54) than the intervention group (5.60, SD 1.90). There were no significant differences in team performance measures between the groups. Study findings indicate use of colored labeled smocks during CPR emergencies resulted in: shorter times to start emergency CPR; reduced errors; more technical skills completed successfully; and no differences in team performance. 
INTRODUCTION

Significance of the Study................................................

Medical errors......................................... 1

Medical errors during emergencies ......................... 2

Interventions used to reduce medical errors .......................... 4

Simulation......................................................... 6

Simulation a potential intervention ....................... 7

The Purpose of the Study ......................................... 9

Research Questions and Hypotheses................................ 9

Theoretical Framework....................................... 10

II $\quad$ LITERATURE REVIEW...................................... 12

Introduction .............................................. 12

Medical errors during emergencies ............................. 12

Identifying medical errors during CPR .................... 16

Preventing medical errors.................................... 25

CPR Performance....................................... 27

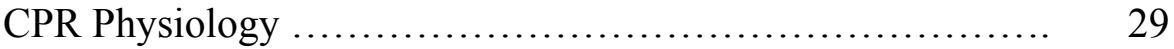

Evolution of CPR .................................... 30

Resuscitation errors ........................................ 32

Quality of CPR ....................................... 34

Interventions used to identify and reduce errors .................... 36

Color coding ................................................. 37

Simulation ....................................................... 39

Evolution of simulation .................................. 40

Current use of simulation ..................................... 42

Simulation effects on behavior and attitudes ................. 43

Barriers to simulation ...................................... 45

Team training .................................................. 49

Deficiencies in team training ............................. 52

Conclusion ................................................ 59

III $\quad$ METHODOLOGY.............................................. 65

Research design ............................................ 65

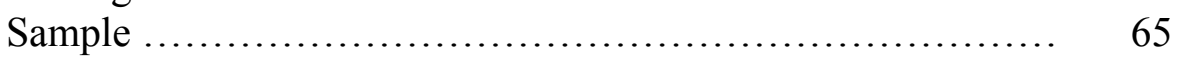

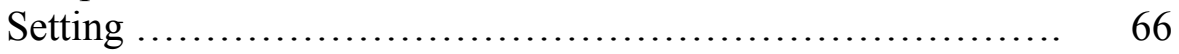

Procedures ............................................... 67

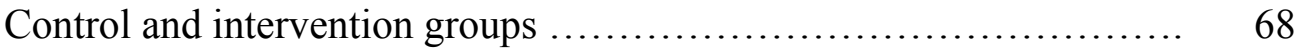

Intervention (smock) group .................................... 70

Scenarios .................................................... 71

Instruments .................................................... 72

CPR scenario technical skills assessment score sheet .......... 72

Time to implement technical skills ............................. 72 
Medical error information ............................... 73

Team performance .................................... 74

Demographic data .................................... $\quad 74$

Data collection .................................... $\quad 75$

Data management ................................... $\quad 75$

Data Analysis ....................................... 76

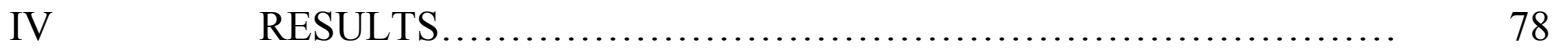

Sample ........................................... 78

Research question number one .......................... $\quad 86$

Research question number two .......................... 87

Research question number three ........................... $\quad 90$

Research question number four .......................... 92

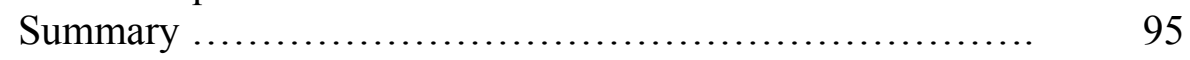

V DISCUSSION AND CONCLUSION ........................... 98

Characteristics of nurses in the sample .......................... 98

Employment and education ............................... 99

Certification ............................................. 99

CPR performance measures .................................... 101

Compressions …........................................ 101

Ventilations ............................................ 102

Medication administration .............................. 103

Defibrillation ............................................. 104

Medical errors …........................................... 105

Code errors ................................................ 106

Technical skills .............................................. 107

Team performance skills ..................................... 108

The use of color labeled smocks during codes...................... 109

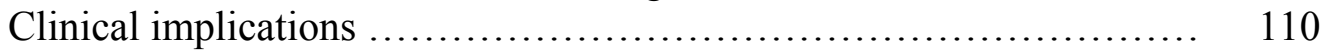

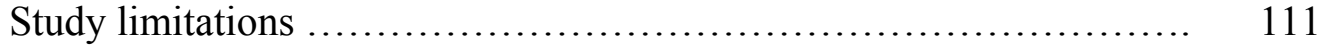

Implications for future research .............................. 111

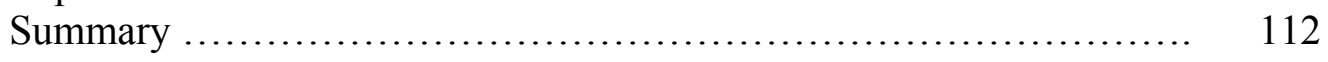

LIST OF REFERENCES ................................................ 114

APPENDICES ...................................................................... 116

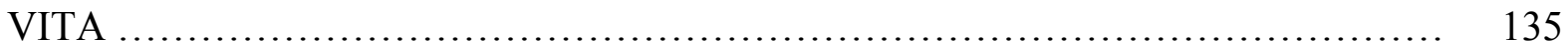




\section{LIST OF TABLES}

Table

1. Demographic and Sample Characteristics $(N=260) \ldots \ldots \ldots \ldots \ldots \ldots \ldots \ldots \ldots . \quad 81$

2. Self reported CPR skill strengths and weakness ............................. 83

3. Time in seconds to implement primary emergency measures $\ldots \ldots \ldots \ldots \ldots \ldots \ldots . \ldots 5$

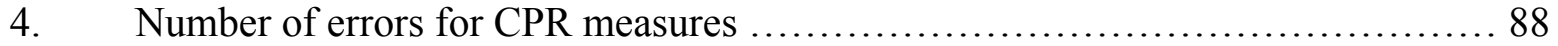

5. Technical skills CPR performance scores............................. 90

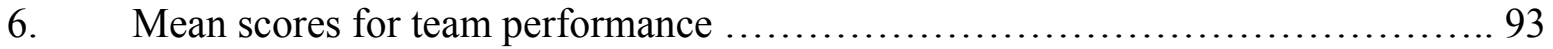




\section{Chapter I}

\section{Introduction}

Nearly 100,000 people in the nation die, and approximately 1.3 million people are injured annually due to preventable medical errors (IOM, 2000) defined as failure of a planned action to be completed as intended or the use of the wrong plan to achieve an outcome. Medical errors occur 39 times more frequently during emergencies. Nurses, the largest group of healthcare workers, may account for nearly 55\% of medical errors in hospital settings (CAPS Link, 2003). To date, few initiatives have shown a reduction in preventable medical errors (Watcher's, 2010). One of the most promising strategies may be the use of simulation technology.

\section{Significance}

\section{Medical Errors}

Most error reporting monitoring systems have identified falls, wrong-site surgery, complications related to anesthesia, and negligent behavior (omission) by healthcare providers as some of the most common errors that occur in U.S hospitals. Due to complex systems used to administer medication, the potential risk of medication errors is greater than all of the common errors combined. Also, Clarke and Aiken, (2002) study suggest that poor nurse staffing increases the frequency of medical errors because over worked nurses are not able to detect potential complications early and activate hospital resources quickly. Thus, implying that failure to rescue patients in hospital settings was associated with high patient/nurse ratios, increase mortality, nurse burnout, and job dissatisfaction among nurses.

In a prospective observational study, researchers' suggest that needless deaths were prime examples of the need for more nurses at the bedside (Aiken, Clarke, Sloane, Sochalski, and Silber, 2002). In their article "Failure to Rescue" patients in hospital settings were more likely to 
experience a medical error due to high patient/nurse ratios and nurses' inability to detect potential complications early to activate the hospital's emergency response system. Aiken and colleagues (2002) research study suggest that patient mortality, nurse burnout, and job satisfaction were strongly associated in hospitals with high patient to nurse ratios. This research study also implies that hospitals may decrease adverse events if they maximize patient care delivery with optimal patient/nurse ratios of $4: 1$. Although, medical errors may never be eliminated, healthcare workers and organizations can create safeguards to reduce errors and minimize their effects especially during emergencies (Aiken et al., 2002).

\section{Medical Errors during Emergencies}

Code- related errors may pose a greater risk of physical harm or death when compared to patients with similar diagnosis and conditions receiving care in non-emergency situations (Lipshutz, Morlock, Shore, Hicks, Dy, Pronovost, and Winters, 2008). Changes that start at the policy level may help sustain patient safety initiatives that reduce patient harm and identify system weaknesses that allow these errors to occur. If code related errors are not corrected immediately, the risk for harm to similar patients increases significantly (Lipshutz et al., 2008). Even though corrective actions may be in place, medical errors may still occur due to reduced staffing, complex care required by patients, and advanced knowledge of technology for new medical devices.

Lipshutz and colleagues (2008) research analysis of emergency code-related errors in 29 hospitals revealed that they are 39 times more likely to result in patient harm than none-code related errors. In this study evaluating 842 errors, $94 \%$ occurred in general community hospitals, $70 \%$ occurred on a general patient ward, and $8.2 \%$ occurred in the emergency department (Lipshutz et al., 2008). Most errors occurred during medication administration, and the most 
common error was omission. Improper dose error accounted for $9 \%$ and unauthorized drug administration accounted for $5.7 \%$ of the errors in this study cohort. The report showed that errors most frequently involved registered nurses (45\%) and respiratory therapists (43\%) and were usually discovered by these two groups (Lipshutz et al., 2008).

This report listed a cause of error in which $30 \%$ were associated with performance (human) deficit, $16 \%$ with work flow disruptions, and $12 \%$ with lack of adherence to a procedure protocol (Lipshutz et al., 2008). Although, all reports listed the code situation as a contributing factor, the most common additional contributing factors were; emergency situation, work load increase, and insufficient staffing. These findings suggest that there is a need for strategies to reduce errors during emergency situations in an effort to improve medication safety.

Although, there has been significant improvements in patient safety measures to reduce medical errors in hospital systems, only a few initiatives actually have shown a reduction in preventable medical errors (Watcher's, 2010). Some patient safety initiatives that have shown progress for hospitalized patients are the use of computerized physician order entry systems (CPOE), using checklists and time outs prior to the start of any procedure, and simulation training (Gaba, 2004).

Advances in simulation technology and training may provide a potential solution for preventing medical errors committed by healthcare providers, and may reveal latent errors hidden in medical organizations (Issenberg \& Scales, 2007). Therefore, simulation training may be one of the most promising interventions used to reduce medical errors by providing programs with the ability to recreate errors in an effort to prevent future occurrences. 


\section{Interventions used to Reduce Medical Errors}

The United States Pharmacopeia Center for the Advancement of Patient Safety (USPCAPS) reports that approximately 1.3 million people are injured annually as a result of medication errors (CAPSLink, 2003). These errors may occur at any stage within the medication preparation process (prescribing, ordering, and administering), resulting in either benign or catastrophic outcomes related to omission of a dose, wrong dose, wrong patient and/or wrong route (Armutulu, et al, 2003).

Medical mistakes usually occur when multiple factors come together to allow them to happen, and nurses may account for nearly $55 \%$ of medical errors in hospitals since they are the largest group of healthcare workers (CAPS Link, 2003). Therefore, nurses play a vital role in creating patient safety initiatives by using teaching strategies like simulation to improve outcomes. Although medical errors were found to occur 39 times more frequently during emergencies, one in five patients continues to suffer from a medication error during routine care. These errors occurred more frequently when nurses were either interrupted or distracted while preparing medications or just prior to performing a procedure.

In a prospective study Pape and colleagues used an innovative teaching strategy to reduce errors during medication preparation and administration when nurses wore a red vest and used a checklist throughout the medication administration process (Pape, Guerra, Muzquiz, Bryant, Ingram, Schanner, Alcala, Sharp, et al, 2005). They demonstrated that medication errors during critical moments can be reduced by $70 \%$ when distractions and interruptions were eliminated prior to and during medication administration (Pape et al., 2005). This innovative strategy resulted in nurses making fewer mistakes and they were less likely to forget to administer 
medications while having sufficient time to identify the right patient, right route, and right dose (Pape et al., 2005).

Hospitals and pharmaceutical companies have joined forces in an effort to correct some latent system errors related to medication mishaps by: changing containers on lookalike drugs, color coding common sounding medications, and building less faulty housing and dispensing systems (Institute for Safe Medication Practice ISMP, 2006). Although these safety measures are practiced in many large hospital organizations, medication errors may still occur due to human factors of omission and commission. These acts of omission may result in new injuries, aggravate old injuries, or ultimately lead to death and unnecessary suffering for patients and family members (Giles and Armon, 2009).

Recent studies have shown that medications were usually omitted when pharmaceutical products were not readily available at the required time of administration, or if the original order was written incorrectly and needed additional clarification prior to administration (Scott, Roger, Wei-Ting and Zhang, 2006). Thus, suggesting that nurses and other healthcare providers need additional training in situational awareness to identify potential problems associated with patient's condition and the use of simulation may improve their clinical performance (Scott et al., 2006).

Since nurses are the largest group of healthcare providers in the health care industry it is of most importance to target this group first to obtain buy-in from the majority. Once nurses are onboard with using simulation and smocks to improve CPR performance measures, physicians and other healthcare providers will most likely join without reluctance. 


\section{Simulation}

Simulation of real life circumstances can be performed with standardized patients or life size simulators to improve communication skills, and allows the learner to make mistakes during training exercises of healthcare's most critical systems using computer-based technology to improve performances. Computer-based simulation programs provide a valuable dimension to healthcare training.

Simulation is a realistic and economical set of tools used to improve and maintain the skills of healthcare providers (Seropian, 2003). Advances in simulation technology have widened the boundaries for increased patient safety measures using a multidisciplinary approach. Handson training helps individuals develop insight from practicing in realistic environments (Seropian, 2003). Therefore, widespread simulation use in medical settings may be the single most effective tool in patient safety training to reduce medical errors (Savoldelli, Naik, Hamstra, and Morgan, 2005).

An observational study to determine the incidence of medication errors during resuscitation in a pediatric department revealed technical skills and knowledge deficiencies during simulation training (Kozer, Seto, Verjee, Parshurum, Khattak, Koren, and Jarvis, 2004). Twenty physicians and fifteen nurses participated in this study. The results revealed errors with incomplete orders, dosing errors, and errors in medication preparation and administration. Eight mock resuscitation scenarios (non-responsive 4 month old in shock related to sepsis; 3 week old with seizures/cardiac arrest; 6 year old with fractures/tachycardia; 2 year old upper airway obstruction/respiratory failure; 3 month old wheezing/respiratory failure; 4 years old in status epilepticus/ respiratory failure; 3 year old respiratory distress/decreased level of consciousness;

and 13 year old in cardiopulmonary arrest) were conducted during routine emergency department 
educational rounds by an experience emergency physician (Kozer et al., 2004). The scenarios included an age appropriate mannequin, a description of the patient's condition, and all necessary clinical data used to make a diagnosis (Kozer et al., 2004).

The resuscitation team consisted of a senior resident, at least two assisting physicians, and two or three pediatric nurses. A full resuscitation was expected, and all members of the team were to respond to the mock code as if they would in a real patient situation. The use of standard references such as the hospital formulary, resuscitation cards, handheld computers, and calculators were allowed. The study results revealed that participants initiated 125 orders for drugs and in 24 of the cases the orders were repeated more than once (Kozer et al., 2004). In 12 cases the ordered drug was not administered.

Also, in 21 cases, the dose was not specified in the order, and in 52 orders the route for administration was not specified (Kozer et al., 2004). This study identified frequent and potentially serious medication errors which occurred at all stages of resuscitation (Kozer et al., 2004). Although, various strategies have been suggested to reduce drug errors including; the use of computer physician order entry systems (CPOE), having clinical pharmacists review orders, and the use of unit dose systems implementation has proven difficult. However, these strategies may not be practical during resuscitation (Kozer et al., 2004) but can be recreated or mimicked using a simulated environment.

\section{Simulation a Potential Intervention}

In an observational study, researchers evaluated the effectiveness of high fidelity simulators (more complex scenarios requiring critical thinking and a high degree of technical skills), on medical-surgical registered nurses' ability to recognize and respond to clinical emergencies (Buckley and Gordon, 2010). Fifty nurses took part in the simulation study, and 38 
(76\%) participated in the follow-up survey three months post training workshop. Of the nurses who responded to the survey, the average age was 35 years; and $90 \%$ were female with an average of nine years of clinical experience (Buckley and Gordon, 2010).

The workshop consisted of 14 hours of lectures related to clinical emergencies, two classes (3 hours each) practicing emergency technical skills (airway management, compressions, checking for pulses, etc) using a Resusci-Anne simulator. Additionally, team-building and communication exercises were included prior to the high fidelity simulation to exposed participants to a variety of combinations related to emergency situations (Buckley and Gordon, 2010).

The results of the Buckley and Gordon (2010) study revealed that within three months following the workshop, participants reported 164 clinical patient events requiring early assessment and immediate intervention. These patient events included 33\% airway or breathing problems, $26 \%$ hypotension crisis, $13 \%$ cardiac rhythms or rate disturbance, $11 \%$ altered level of consciousness, 9\% chest pain, 7\% cardiac arrest, and 5\% electrolyte disturbance (Buckley and Gordon, 2010). The participants reported that the most useful aspects of the simulation workshop in assisting in their ability to respond to real life emergencies were; debriefing after scenarios $(87 \%)$, learning assertiveness skills $(80 \%)$, and getting good patient hand offs (53\%)(Buckley and Gordon, 2010).

Overall, the results showed that both technical and non-technical skills acquired during simulation exercises were relevant in real life emergencies, and nurses reported using these skills 3-5 times within the first three months post training (Buckley \& Gordon, 2010). This research study also suggests that the use of immersive simulation (physically involved in believable patient cases) combined with classroom teaching, improves medical-surgical perceived ability to 
respond to patient clinical emergencies. The simulated environment may mimic real experiences with planned ones to evoke specific aspects of a real clinical setting (Buckley and Gordon, 2010).

In summary, hundreds of thousands of hospitalized patients die or are injured annually in the US due to medical errors. The risk is amplified in emergency situations. Establishing safety systems to prevent patient harm is essential in health care in the U.S. While pharmaceutical companies and some hospital systems have been improved to reduce medical errors, interventions to reduce errors due to human factors have shown less advancement. Simulation technology offers a venue to test health care provider practices prior to implementation and potentially assist providers in completing tasks as intended resulting in desired outcomes. The proposed experimental study investigating the use of a color-coded smock system that identifies nurses' roles during an emergency situation (CPR) in a simulated environment is one intervention that may potentially protect the safety of patients during emergencies.

\section{Purpose and Research questions}

The purpose of this quasi experimental study is to test a color-coded SMOCK system on time to implement emergency patient care measures, team performance, and medical errors committed during simulated emergencies. Comparing control and intervention (smock) groups during emergencies (codes), will there be a difference in:

1. Time to implement ventilation, compressions, medication administration and defibrillation.

2. The number of recorded medical errors (i.e. sequence in initiating ventilation and compression; medication administration [giving the wrong medication via the wrong 
route,]; omission of ventilation, compressions, requested medications or defibrillation; or errors in providing ventilation, compressions, or defibrillation).

3. Performance of Technical Skills (mean scores for ventilation, compression, medication administration, defibrillation, and total).

4. Team performance (problem solving, situational awareness, leadership, resource utilization, and communication).

\section{Theoretical Framework}

Donabedian's classic paradigm to assess quality of care is based on a three component approach; structure, process, and outcomes in which the model proposes that each component has a direct influence on the next. Structure refers to the attributes of the settings in which providers deliver health care, including material resources (e.g., electronic health records), human resources (e.g., staff expertise), and organizational structure (e.g., hospitals vs. clinics). The process refers to the actual care the patient receives while in a medical facility and health outcomes are the direct result of a patient's health status as a consequence of contact with the health care system.

Donabedian's model was chosen to guide the theoretical framework of this study by evaluating the environment in which medical errors are more likely to occur and improving outcomes through changing the process in the delivery of safer emergency care. Since nurses contribute to the chaotic environment in which medical emergencies occur, their technical skills and team performance will be evaluated as part of the care management process during cardiopulmonary resuscitation. The standard practice of care during CPR will be compared in two groups to evaluate a safer process of care (using smocks to identify roles) which may eliminate potential medical errors and improve patient outcomes. 


\section{Donabedian's Model}

Structure $=$ Hospital

Emergencies (mock codes) in a simulated setting
Process $=$ CPR performance in two groups of Nurses, Control \& Intervention (Smocks)
Time to implement ventilation, compressions, medication administration and defibrillation

Number of recorded medical errors, sequence in initiating, ventilation and compression; medication administration, and giving the wrong medication via the wrong route; omission of ventilation and compression, requested medication or defibrillation

Performance of technical skills (mean scores for ventilation, compression, medication administration, defibrillation and total.

Team performance (problem solving, situational awareness, leadership, resource utilization, and communication) 


\section{Chapter II}

\section{Introduction}

Approximately 1.3 million people are injured every year because of medical errors. Some situations including emergencies using resuscitative measures may result in a greater number of errors. According to the National Registry of Cardiopulmonary Resuscitation (NRCPR) in 2007, nearly 21,748 cardiac arrests were reported, and the rate of survival to discharge after in-hospital cardiac arrest was 27\% among children and 18\% for adults (American Heart Association, 2008).

The low rate of survival after a cardiac arrest can be attributed to a number of factors including poor performance of cardiopulmonary resuscitation measures (Abella et al., 2004). The quality of life support during cardiopulmonary resuscitation (CPR) directly affects outcome (Hunt, 2008). Due to the emergent nature of the situation, little time is available for providers to think through or discuss options of care (Abella et al., 2004).

Recent studies suggest that medical errors during emergencies are 39 times more likely to result in harm, and hospital mock code drills have shown that at least one resuscitation error and miscommunication among care givers occurred at each drill (Lipshutz et al., 2008; Armutulu et al., 2008). These studies also imply that human factors (i.e. a stressful and chaotic environment, conditions of cardiac arrest, weak chest compressions, rescuer fatigue, and infrequent CPR recertification classes) may be the greatest contributor to poor patient outcomes in-hospital cardiac arrest. This chapter will focus on medical errors during emergencies and interventions used to identify and reduce errors through the use of simulation technology.

\section{Medical Errors during Emergencies}

Despite ongoing patient safety initiatives and widespread attention related to medical errors since the 1999 IOM report of 98,000 annual deaths, it is still difficult to develop and 
implement interventions to reduce medical errors during emergencies. Studies have examined the association between errors and the environment (systems), but few have actually created interventions that significantly reduce medical errors in a hospital setting (Crawford, Cohen, and Tafesse, 2003).

In US hospitals, the frequency of medical errors have been associated with complex healthcare systems, poor policy designs, and human factors including ergonomics (Leape, 2002: Crawford et al., 2003). A national medication error database found that 11,000 errors were reported by 484 emergency departments throughout the US over a five year period (Santell et al., 2003). In the report, some contributing factors associated with medical errors in the emergency department were incorrect medication administration, delays in diagnosis and treatment, and faulty medical devices (Santell et al., 2003). Additional studies also report that medication errors occurred in one out of every five prescribed medication doses; and suggest that errors often occur because of high noise levels, distractions, interruptions, ineffective communications, lack of focus, and poor team work (Barker et al., 2002; Pape et al., 2003).

In an observational study, the effects of a standard protocol and visible signage in a hospital setting were examined to improve medication delivery (Pape, Guerra, Muzquiz, Bryant, Ingram, Schanner, Alcala, et al., 2005). The standard protocol and visible signage was used to improve mental focus, standardize medication delivery practice, and reduce distractions among nurses when preparing or administering medication. Seventy eight nurses on medical wards were randomly observed by the unit educators and charge nurses during medication delivery times between 7:00am and 7:00pm and special attention was made during the routinely scheduled medication delivery times (8:00am, 10:00am, 12:00pm, 2:00pm, or 4:00pm) (Pape et al., 2005). 
Nurses on five pre-selected units were educated regarding the protocol and the steps to be used during medication administration were provided on small $4 \times 5$ inch checklist cards for ease of reference (Pape et al., 2005). The checklist cards contained the medication steps and were placed on the medication cart or in the pocket of the medication book. After the nurses became familiar with the protocol, signs were posted (do not disturb) above the automated medication dispensing machine, and nurses were asked to use the protocol checklist, avoid conversations, and prevent distractions during medication administration (Pape et al., 2005). The nurses self reported on a ten item medication administration distraction observation sheet that listed the potential distraction sources; physicians, other personnel (nurses), phone calls, other patients, visitors, missing or wrong-dose medications, emergency situations, conversations, computer problems, and external noise.

The study results revealed that $90 \%$ of the nurses printed the medication administration record (MAR) forms and carried them to the patient room, and only $75 \%$ compared medication from the automatic medication dispensing machine with the MAR form (Pape et al., 2005). Additionally, most nurses ( $80 \%$ ) checked the patient allergy band or ID bracelet, while only $38 \%$ told the patient the name of the medication being given. Also, the greatest reduction in distractions after placing signs were distractions caused by other nurses, conversations with someone unnecessarily, and loud noise levels. Nurses in this study also, stated that the physicians and residents interrupted them regardless of whether they were doing something important or routine (Pape et al., 2005).

The study also implies that nurses may commit medication errors because of various challenges associated with completing tasks and using complex medical devices in a demanding environment (Pape et al., 2005). This study also reported delays in treatment related to omissions 
or wrong administration of medication by nurses who were either distracted or interrupted during medication preparation/administration, and nurses' accounted for nearly 55\% of errors committed by healthcare workers (Pape et al., 2005; Lipshutz, 2008).

In Pape's earlier research study on errors, she suggests that most medication errors result from distractions or interruptions among health care workers during critical times of medication administration and preparation (Pape et al., 2003). Typically these errors result from administering medication via the wrong dose, to the wrong patient, using the wrong route, and or by omission of the dose. Pharmaceutical companies have focused mostly on system issues related to medication administration by changing similar containers, color coding common medications, and building less faulty delivery systems (Lipshutz et al., 2008). However, some less expensive ways to prevent medication errors have been described by preventing chaos, distractions, and interruptions during medication preparation and administration (Pape et al., 2003).

Factors associated with medical errors have been linked to the improper use of medical devices or faulty equipment. The Food and Drug Administration (FDA) created safety standards to deter the harmful effects associated with the intended use of a medical device. The FDA suggests that key features and strategies related to medical devices include better design to reduce or eliminate mistakes. Many device-related incidents were linked to poor design and lack of usability testing as seen with cardiac pacing and cardio version devices (FDA, 2004). However, the human-machine interface is more complex as the dynamics between both elements are constantly changing when a device communicates with the user through audible and visual displays. Although, automated external defibrillator systems are created for easier use, these devices can sometimes malfunction and deliver incorrect electrical doses (FDA, 2004). 
Unfortunately, medical emergencies present a complex environment of circumstances that provide a situation where medical errors can easily be hidden or overlooked.

\section{Identifying Errors during $C P R$}

In a prospective study of 34 consecutive hospital mock codes, the median elapsed time to initiate important resuscitation maneuvers in simulated pediatric medical emergencies (i.e., "mock codes") were measured to identify types and frequency of errors committed during the actual event (Hunt, Walker, Shaffner, Miller, and Pronovost, 2007). The study was conducted within a 40-month period, in 1 of 3 urban academic medical centers with pediatric residents.

The mock codes occurred in locations to which the pediatric code team might have to respond, including wardrooms, procedure rooms, and radiology suites. All scenarios were unique to avoid pattern recognition by caregivers attending more than one mock code, and consisted of an unannounced simulated crisis situation involving: respiratory distress or insufficiency, respiratory arrest; hemodynamic instability, and/or cardiopulmonary arrest (Hunt et al., 2007).

The mock code events were started when the code button in a chosen room was activated, indicating that help was needed, and the first person (i.e. nurse, pediatric resident, ward clerk, or respiratory therapist) to enter the room in response to the code button was defined as the first responder. A short vignette was given, and the first responder was told to proceed as though it was a real code (Hunt et al., 2007).

The study results revealed that ward nurses were involved in $100 \%$ of the mock codes as first responders, and in $88 \%$ of theses codes ward clerks and telephone operators were the next individuals to offer assistance. The time it took the first responder to enter the room, be oriented to the exercise, and have the vignette explained was always within 15 seconds. The median time of arrival for any physician was 3 minutes and 6 minutes for the first member of the code team 
(Hunt et al., 2007). Further, analysis of the results showed that in $25 \%$ of the scenarios, the first responders took $>1.5$ minutes to initiate assessment of airway and breathing, $>5$ minutes to initiate bag mask ventilation (BMV), and $>7$ minutes to initiate assessment of circulation by checking the pulse (Hunt et al., 2007).

Results from this study revealed that all of the codes had at least 1 resuscitation error; in $12(71 \%)$ of the mock codes requiring chest compressions, the team failed to adhere to AHA pediatric basic life support (PBLS) guidelines and did not provide immediate chest compressions. Also, in $31(100 \%)$ of the mock codes that required pediatric advance life support (PALS) including the administration of medication, the team failed to adhere to the AHA guidelines. In all of the 6 patients who became apneic as a result of pulseless ventricular tachycardia/fibrillation, the physicians attempted to intubate rather than initiate compressions or defibrillate as soon as possible (Hunt et al., 2007).

This study also highlights the importance of the quality of CPR administration and the timeliness of the nurse's initial actions due to the length of time it takes before a physician or code team member arrives on the scene. Results suggest that nurses in this study were required to complete too many preparatory tasks (e.g. drawing up resuscitation drugs, getting the code cart and defibrillator) resulting in delays in the start of CPR which may ultimately result in poor outcomes. The use of simulation allowed the study team to evaluate and train providers without endangering patients, and uncover deficiencies related to CPR administration in a hospital setting (Hunt, et al., 2007).

In another prospective study by Hunt and colleagues (2009), simulation technology was used to identify delays and errors in cardiopulmonary resuscitation and defibrillation by pediatric residents during simulated cardiopulmonary arrests (CPA). This study hypothesize that errors in 
performing basic chest compressions, and advanced defibrillation (BLS/ALS) are common, and poor quality resuscitation performance can ultimately affect patient outcomes (Hunt et al., 2009). Pediatric residents were enrolled in a prospective observational study using a high fidelity mannequin simulator to determine if they met performance standards set by the 2005 AHA guidelines for pulseless ventricular tachycardia (PVT). Seventy pediatric residents from a tertiary care academic hospital participated in monthly simulated resuscitation training sessions. Videotaped data was reviewed by two reviewers independently to assess the adequacy of the start and stop times for ventilation and compression (Hunt et al., 2009).

The mock codes were initiated by a nurse asking the resident to evaluate a 12 year old child admitted one hour ago from the emergency department diagnosed with frequent premature ventricular contractions (PVC's). The scenario was set for the patient to be responsive for the first minute, and then become unresponsive, apneic, and have a pulseless ventricular tachycardia (PVT) event. The nurses were instructed to assist in the code as usual by following the 2005 AHA guidelines for CPR and only perform specific tasks when asked without leading the resuscitation (Hunt et al., 2009).

The study results showed that $50 \%$ of residents never led a code team during CPR, $9 \%$ did not have PALS training, and 9\% did not have BLS training. Also, $92 \%$ of the residents' recognized apnea and ordered the nurse to assist in ventilation with a median time of 24.5 seconds and 7\% never asked for assistance with ventilation. Sixty six percent of the residents failed to start compressions within one minute of pulselessness, and 33\% never started compressions during the entire scenario. Additionally, $75 \%$ of the residents notice a change in the patient's condition from a normal sinus rhythm to PVT within 9 seconds, and $95 \%$ noticed the change in less than 40seconds. The median time residents asked for the defibrillator was 33 
seconds, however, there was a gap in the time they asked for the defibrillator and when they successfully defibrillated. Only $54 \%$ of residents defibrillated the mannequin in $<3$ minutes of onset of PVT, and of these residents the median time to successfully deliver a shock was 84 seconds (Hunt et al., 2009).

The study results also revealed that $48 \%$ of the residents had at least one unsuccessful attempt at discharging the defibrillator (forgetting to charge the defibrillator), and $83 \%$ made at least one error while operating the defibrillator (turning machine on, removing paddles from machine, changing energy level, charging the defibrillator, and confusion with buttons and cables on the machine) (Hunt et al., 2009). Also, Hunt and colleagues (2009) research study showed that the majority of the residents did not deliver timely compressions or defibrillations according to the 2005 AHA recommendation. These results revealed that $33 \%$ of the residents failed to start compressions and 7\% never defibrillated, virtually guaranteeing a fatal outcome for those patients (Hunt et al., 2009).

This study suggest that pediatric residents do well on cognitive tests of resuscitation knowledge after a PALS course and perform poorly on practical tests of their resuscitation skills. The results of this study also imply that future studies should be performed to determine the decay curve of important technical skills as well as the frequency in which training should be done to help clinicians learn and maintain sharp skills (Hunt et al., 2009).

This study also claimed that many of the residents were not recently (within the two year recertification window) trained according to the 2005 AHA guidelines for CPR, and this may have been the reason for poor technical skills and the lack of applied knowledge (Hunt et al., 2009). Also, asking nurses to perform at a substandard of care during a code could have also 
contributed to prolong timing to initiate required technical skills which do not reflect routine care during CPR.

In this study, nurses were asked to help physicians without taking over the code situation by not suggesting routine procedures to perform CPR measures, and this type of participation by nurses in real life code situation are not typical. Nurses are more inclined to take over the emergency since they are usually the first responders and may typically have more experience than resident physicians in training. The need to observe and improve both knowledge and technical skills during emergencies for physicians, nurses, and other support staff has been substantiated with this study (Hunt et al., 2009).

Other studies also show that healthcare workers in high demand areas suffer from alarm fatigue which affects their reaction to critical alarms by silencing the audible tone or ignoring them altogether (AHRQ, 2005). The noise levels in the hospital environment can also influence the safe and proper use of medical devices. Excess noise may mask safety alarms and contribute to increase stress levels for in-patients and hospital staff (Pape et al, 2005). However, most errors can easily be avoided when healthcare workers are trained to identify potential hazards, circumvent careless mistakes, and follow hospital policies and procedures. Unfortunately, it is difficult to identify medical errors during emergencies because the exact cause of death in these patients may be a mixture of accidental deaths and deaths due to natural causes (AHRQ, 2005).

Although, poor survival outcomes from cardiac arrests have been associated with time of arrival of first responders and advance healthcare teams, recent studies show that outcomes may be closely related to actual performance of cardiopulmonary resuscitation (CPR) measures. A retrospective study using all non-traumatic out of hospital cardiac arrest events during a 12 month period for individuals at least 16 years of age in Tucson Arizona were evaluated to 
identify interruptions of chest compressions during emergency medical systems (EMS)

resuscitation performance (Valenzuela, Kern, Clark, Berg, Berg, Berg, Hilwig et al., 2005). From 2001 to 2002, sixty one out of hospital cardiac arrests were reviewed from individuals treated with a printable automated external defibrillator (life pack 500 and life pack 12 AED that provided a continuous wave form), along with the code arrest record to verify adherence to the AHA 2000 guidelines for cardiopulmonary resuscitation (Valenzuela et al., 2005).

In this retrospective study, survival definitions were determined by review of paramedic records, bystander performance, and emergency medical services (EMS) performance to establish a relationship between interruptions of chest compressions and survival. The study defined arrival time interval to include the time needed to find the patient and begin an initial assessment. Also, arrival to diagnosis was described as the time needed to make a rhythm diagnosis and treatment of either defibrillation or begin chest compressions (Valenzuela et al., 2005). Data were collected from the life pack defibrillator throughout the resuscitation effort until transport began. Data were not analyzed during transportation because of noise artifacts related to motion causing distortion on the cardiac wave form. The Fisher's exact test was used to compare when chest compressions were and were not performed, and a Mann-Whitney U test with an unpaired 2 tailed t-test were performed on parametric data (Valenzuela et al., 2005).

The study results showed that the median arrival time was 6 minutes, to diagnose a rhythm was 13 seconds, and to treat the rhythm with compressions or defibrillation was 20 seconds. Also, time from arrival to a patient in ventricular fibrillation to delivering the first shock was 54 seconds with an additional 30 seconds between the second and third shocks (Valenzuela et al., 2005). Chest compressions were only performed $43 \%$ of the time, because EMS providers were frequently interrupted with other resuscitation tasks (setting up the defibrillator or preparing 
to intubate). The time interval from arrival to first chest compressions were 78 seconds, the longest continuous period of chest compressions were 122 seconds ( 3 minutes), and the shortest period was 11 seconds (Valenzuela et al., 2005).

These study results surmise that CPR measures from EMS services personnel were initiated in a timely fashion, however, since the arrival time was after 6 minutes cardiac compressions should have been started or resumed immediately upon arrival. In 2003, Weisfeldt and Becker clearly demonstrated in animals that the electrical phase in cardiac arrest occurred in the first few minutes of the arrest event where immediate defibrillation would be most effective. Since EMS providers routinely arrived 6 minutes after the start of an event, defibrillation should not have been considered the first treatment of choice. Weisfeldt's and Becker's (2003) animal research also demonstrated that defibrillating the heart during the circulatory phase of ventricular fibrillation, while the heart was at complete rest, resulted in poor survival outcomes.

Valenzuela and colleagues' (2005) study of 61 patients, 21 (34\%) had a true ventricular fibrillation rhythm. Seventeen patients were successfully defibrillated, but all converted to asystole or pulseless electrical activity (PEA) when compressions were not resumed or started. Also, 10 of the 61 patients had a return of spontaneous circulation (ROSC) and were admitted to the hospital alive; however, only four patients (7\%) survived to hospital discharge. Additionally, eight of the ten patients collapsed in front of witnesses, received bystander CPR, and had an initial VF rhythm. Thus, the results in this study suggest that witnessed cardiac arrests have better outcomes but poor CPR performance may contribute to low survival rates (Valenzuela et al., 2005).

According to the American Heart Association 2010 CPR guidelines, the first five minutes of CPR performance is crucial for optimal survival. Suboptimal chest compressions may 
greatly contribute to poor survival outcomes (AHA, 2010; Valenzuela et al., 2005). Although patient survival rates documented in Valenzuela et al (2005) study is low and can be attributed to out of hospital delays, historical rates of in-hospital survival after cardiac arrest are not much better with adult survival rates of 18\% (Valenzuela et al., 2005; Lipshutz et al., 2008). These low rates of survival may be a direct result of technical performance related to device utilization as the use of automated external defibrillators (AED) increased resuscitation interruptions.

These interruptions were also related to other resuscitation tasks such as; reassessments of circulation, intubation, and intravenous line placement which too may minimize chest compressions. More research is needed to identify strategies to reduce interruptions during resuscitation so hemodynamic support may be maximized for an overall improvement in patient survival regardless of where the arrest occurs (in or out of hospital).

A clinical observational study revealed that rescuers consistently hyperventilated patients during out of hospital CPR which resulted in poor survival outcomes (Aufderheide, Sigurdsson, Pirrallo, Yannopoulos, McKnite, Briesen, Sparks, et al., 2004). The research study took place in the city of Milwaukee where a physician and a paramedic (research team) were dispatched to the scene of each patient in cardiac arrest. In this study, patients met entry criteria if they were twenty one years of age or older, believed to be in cardiac arrest, and were successfully intubated with an endotracheal tube undergoing CPR at the time of arrival of the research team (Aufderheide et al., 2004). As part of the research, the team connected a noninvasive intrathoracic pressure sensor between the endotracheal tube and the bag valve resuscitator. The ventilation frequency, duration, and percentage of time in which a positive pressure breath was recorded in the lungs were then calculated with a digital caliper (Aufderheide et al., 2004). 
In Milwaukee the EMS system uses both paramedics and emergency medical technicians (EMT's) to manage patient's airway and provide rescue breathing during out of hospital treatment for cardiac arrest. These medical professionals must continuously remain certified in BLS skills and demonstrate acceptable performance of CPR to maintain their licensure. The professional rescuers must also attend CPR refresher courses every two years. Additionally, all paramedics and EMT's were trained and demonstrated CPR performance according to the AHA guidelines prior to the start of the study.

The first seven patients enrolled in the study made up group one and after the research team recognized that rescuers were consistently hyperventilating ( $>30$ breaths/minute) patients in cardiac arrest, they were retrained immediately to provide 12 breaths per minute. The retraining classes or techniques were not clearly described in this study. After retraining rescuers, the research team enrolled another six patients in the study which comprised group two. A total of 13 patients were enrolled into the study ( 6 women and $7 \mathrm{men}$ ), and they had a mean age of 63 years (Aufderheide et al., 2004).

The study results revealed that ventilation rates were significantly higher than AHA recommendations for all thirteen patients. Also, after retraining the rescuers, the rate of delivering breaths decreased (still slightly higher than 12 breaths per minute), but the duration of ventilation was prolonged in group two. The percentage of time which positive pressure was recorded in the airway was not significantly different between groups. No patients in this study survived to hospital discharge.

To further investigate these findings, Aufderheide and colleagues performed a hemodynamic study using pigs. The results of this study showed that an increased ventilation rate was associated with significantly higher mean intrathoracic pressures $(p<0.0001)$ and 
significantly lower coronary perfusion pressures ( $\mathrm{p}=0.03$ ) (Aufderheide et al., 2004). The survival rate of pigs ventilated at 12 breaths per minute with $100 \%$ oxygen was 6 of $7(86 \%)$, compared with the survival rate of 1 of $7(17 \%)$ pigs ventilated at a rate of 30 breaths per minute with $100 \%$ oxygen, and 1 of $7(17 \%)$ pigs ventilated at a rate of 30 breaths per minute with $95 \%$ oxygen and $5 \% \mathrm{CO}_{2}$ (Aufderheide et al., 2004).

These study results suggest that hyperventilation during CPR may contribute to poor outcomes, and rescuers often deliver more than double the number of artificial breaths recommended by the AHA. The study findings also show that both rapid rates with short durations of ventilations were just as bad as slow rates with long durations of ventilation because they contributed to high pressures in the chest (Aufderheide et al., 2004). The data from this study also demonstrates that any incidence of hyperventilation is likely to have detrimental hemodynamics and survival consequences due to low flow rates in cardiac arrest patients (Aufderheide et al., 2004).

EMS personnel were all trained using the AHA guidelines for CPR in a classroom setting, actual performance in the field demonstrated deviances and poor survival outcomes. The results from this study further validate the poor quality of CPR performed by highly trained medical professionals (Aufderheide et al., 2004; Hunt et al., 2009).

\section{Preventing Medical Errors during Emergencies}

During medical emergencies, every second counts as patient harm increases and survival decreases. Medications administered during cardiopulmonary arrests (codes) are considered high risk drugs which can prove fatal when given inappropriately or by mistake (Kozer et al., 2004). Code-related medication errors are 51 times more likely to result in death than non-code related errors, and most often result from erroneous medication calculations, miscommunications, 
protocol deviation, knowledge deficit, and or a device dispensing error (Kozer et al.,2004; Lipshutz et al., 2008).

Henneman and colleagues, (2010) exploratory study described error recovery strategies used by critical care nurses to identify, interrupt, and correct errors. In this study twenty nurses were recruited from a convenient sample of five critical care units in the US. All nurses had at least 6 months of critical care experience.

Focus groups were used to explore medical error prevention strategies among 20 nurses during seven 60 to 90 minute sessions. These nurses had a median of 12 years experience in critical care. The questions presented in the focus groups were reviewed for content validity by five experts (two critical care nurse clinicians, a patient safety physician, and 2 research nursescritical care and patient safety), and all sessions were audio taped. The content of the tapes were independently analyzed by five reviewers to determine patterns and themes that summarized strategies for identifying, interrupting, and correcting errors (Henneman et al., 2010).

The results of this study describe the pivotal role nurses play in identifying medical errors using eight strategies: knowing the patient, knowing the "players", knowing the plan of care, surveillance, knowing policy/procedures, double checking orders, using systemic processes, and asking questions. Nurses also used three strategies to interrupt errors by; offering assistance, clarifying orders, and verbally interrupting situations that had a high potential for an adverse event. Nurses used six strategies to correct or prevent errors during all phases of the error recovery process by: persevering to meet the patient's need, being physically present during any emergency, reviewing or confirming the plan of care, offering options to improve care, referring to standards (protocols) or experts, and asking for assistance from another nurse or physician when necessary (Henneman et al., 2010). 
These error prevention strategies have been identified in the action of both critical care nurses and emergency room nurses suggesting that nurses in these two groups prioritize the use of strategies according to their patient's needs. However, identifying errors during emergencies and finding solutions to correct them are difficult because of many technical factors and poor access to verifying pertinent patient information while in an active code situation (Kozer et al., 2004; Hunt et al, 2009).

Technical factors such as code location, scarcity of equipment, and unfamiliar team members may result in total environmental confusion, poor communication between team members, and other organizational factors that may contribute to collateral damage (damage to other patients not involved in the code) (Lipshutz et al., 2008). Although codes may last only a few minutes, care to other patients may be interrupted for at least an hour. Since individuals on the rescue team are summoned from various areas of the hospital when a code blue emergency is announced, many patients may be left unattended or have an interruption in continuity of care resulting in unmet needs (Aiken et al., 2002; Henneman et al., 2010).

The emergency room is a fast paced, high stress environment, and many patients may suffer harm due to medical mistakes which may or may not be preventable or avoidable. Although, hospitals may minimize the risk of errors by implementing standard policies and procedures, errors during emergencies may still occur due to many unforeseen circumstances that lead to lack of treatment, inappropriate treatment, over treatment, and misdiagnoses especially during cardiopulmonary resuscitation (Kozer et al., 2004; Lipshutz, et al., 2008).

\section{CPR Performance}

Cardiac arrest (codes) occurs in individuals of all ages. Cardiopulmonary resuscitation $(\mathrm{CPR})$ is an emergency procedure which is performed in an effort to manually preserve brain 
function until further measures can be taken to restore spontaneous blood circulation and breathing. The body may suffer irreversible damage after four to seven minutes once blood flow has stopped and oxygen has not been transported to the heart or brain. After eight minutes without oxygen, the heart cannot maintain a normal rhythm and further decreases the chances of survival or significant brain injury as brain cells began to die (AHA, 2010). Therefore, effective CPR enables enough oxygen to reach the brain to delay brain death and allows the heart to remain responsive to electrical stimulation (cardio version) procedures (AHA, 2010).

Literature on code-related errors is scarce, and information on code-related errors affecting patients other than the one involved in the code is largely unavailable (Lipshutz et al., 2008). The ideal solution to the problem of code-related medication errors is the prevention of code situations altogether, although, complete elimination of medical emergencies for cardiopulmonary resuscitation is not a realistic goal. Reducing the frequency of code situations is potentially feasible as great strides are made to improve training to where CPR performance skills are sustained over time (Lipshutz et al., 2008).

In one research study, the authors suggest that highly trained hospital personnel often fall short of following the intended cardiopulmonary resuscitation (CPR) 2000 AHA guidelines during a medical emergency (Abella, Sandbo, Vassilatos, Alvarado, and O’Hearn, 2004). Unfortunately, timing, improper ventilation, and inadequate rate and depth of compressions are likely causes of poor performance that limits the effectiveness of CPR. Although the AHA continues to update the guidelines for CPR administration every five years, it has still proven difficult to adequately measure the performance and outcome of resuscitation procedures (Abella et al., 2004; Lipshutz et al., 2008). 


\section{Cardiopulmonary Resuscitation Physiology (CPR)}

CPR is indicated for any person who is unresponsive with no breathing, or who is only breathing with occasional agonal breaths. CPR involves chest compressions at least $5 \mathrm{~cm}$ deep and at a rate of at least 100 per minute in an effort to create artificial circulation by manually pumping blood through the heart (Weisfeldt and Becker, 2003; Aufderheide et al., 2004; Hunt et al., 2007). In addition, the rescuer may provide breaths by either exhaling into the patient's mouth or utilizing a device that pushes air into the lungs (AHA, 2010). CPR alone is unlikely to restart the heart as its main purpose is to restore partial flow of oxygenated blood to the heart and brain (Weisfeldt and Becker, 2003). The main objective of CPR is to delay tissue death and to extend the brief window of opportunity for a successful resuscitation without permanent or significant brain damage (Aufderheide et al., 2004). CPR is continued until there is a return of spontaneous circulation (ROSC) or the person is declared dead by the attending physician or other medical personal.

Cardiac arrest/failure occurs when the heart cannot provide enough blood for adequate perfusion to the coronary and cerebral arteries. This circulatory failure may result from decreased muscle contractility or arrhythmias that allow insufficient time for diastolic filling of the ventricles causing asynchrony; stressing the circulatory system by increasing after load of the heart resulting in valvular dysfunction, tamponade, and or muscle ischemia (Babbs, 2009).

Circulatory failure caused by hypoxia, lactic acid or other by products of anaerobic metabolism may result in decrease blood flow to the heart and brain, also causing a decrease in blood flow to other organs (skin, muscles, intestines, etc,) (Peniel and Benowitz, 1984). Research studies have shown that cardiac output during resuscitation is compromised with mean arterial pressures falling less than $50 \%$ of normal in humans and $30 \%$ in animal models (McDonald, 
1981). Cardiac arrest is usually caused by fatal arrhythmias such as ventricular tachycardia/fibrillation where the heart beats are fast but palpable, and the heart beats deteriorates until they are no longer detectable (pulseless electrical activity) or present (asystole). The physiology of cardiac arrest is complex; however the treatment for sudden arrest using the AHA recommendations for CPR performance measures may be quite simple and effective if performed correctly.

Evolution of CPR

Dr. James Elam was one of the first physicians to demonstrate experimentally that cardiopulmonary resuscitation (CPR) was an effective technique to revive victims of sudden cardiac or pulmonary arrests (AHA, 2010). Also, in the late 1950's Dr. Peter Safar along with Dr. Elam wrote the book for the modern day CPR, and pioneered the development of the ABC's (airway, breathing, and circulation) for resuscitation (Cummins, Ornato, Thies, and Pepe, 1991; AHA, 2010). Dr. Safar conducted research on existing basic life support procedures including controlling a person's breathing airway by tilting back his or her head with an open mouth; and using mouth-to-mouth breathing. He combined these techniques with a procedure known as closed-chest cardiac massage (chest compressions) to become the basic life support method for CPR (Cummins, et al 1991; AHA, 2010).

Dr. Safar collaborated with Ausmund Laerdal (a toy maker) and created the first medical simulator (a doll like patient model, resusci-Annie) in 1960 to aid in the teaching of emergency basic life support (BLS) and training of lay people (Cooper \& Taqueti, 2004; AHA, 2010). In 1974 the American Heart Association (AHA) and the National Research Council (NRC) established the standards for cardiopulmonary resuscitation (CPR) using resusci-Annie as a model for hands-on experience (AHA, 2010). This mode of teaching provided national 
recommendations for resuscitation and established the first AHA guidelines for CPR in the U.S. (Cooper and Taqueti, 2004; AHA, 2010).

The AHA guidelines state that someone should call for help by activating the emergency medical-services system in the community (by calling 911 in most U.S. cities) or the code team in the hospital (AHA, 2010). The steps of CPR begin with initiating cardiopulmonary resuscitation until advanced help arrives; assess the heart rhythm and defibrillate the heart if indicated; and administer medications and protect the airway when necessary. This sequence (rapid access, rapid cardiopulmonary resuscitation, rapid defibrillation, and rapid advanced care) is termed the chain of survival (Cooper \& Taqueti, 2004). Dr. Elam and colleagues suggested that the powerful tools and techniques of cardiac resuscitation can benefit thousands of people in the community and hospital setting, conversely, when inappropriately applied, cardiac resuscitation may result in human suffering (Cummins et al., 1991).

The first 1975 AHA CPR guidelines for lay people, included specific instructions for resuscitating adults, children, and infants using the ABC's (airway, breathing, and circulation) as described by Elam and colleagues. The resuscitation procedure included: checking for responsiveness, calling for help, tilting the head back to open the airway, looking and listening for air exchange, checking for pulses, and starting compressions (Cummins et al., 1991; AHA, 2010). The same technical steps are performed for infants and children; however, the depth and rate of compressions and ventilation are adjusted for effectiveness and safety in younger populations (Cummins et al., 1991; AHA, 2010).

In 2010 the AHA recommended a major change in the procedures for CPR since its inception because animal studies have shown that brief pauses in chest compressions adversely affect hemodynamics during resuscitation (Weisfeldt and Becker, 2003; Aufderheide et al., 2004; 
AHA, 2010). These studies have shown that the effectiveness of chest compressions is dependent upon the rate, depth, pressure, and the technique to generate sufficient flow that improves perfusion (Weisfeldt and Becker, 2003). The new (2010) CPR guidelines recommend that compressions begin as soon as possible at a rate of 30/2 (compressions to ventilations). These new recommendations reversed the old guidelines of 2 ventilations before every 15 chest compressions to assure immediate and continuous external cardiac message (AHA, 2010).

Although, the guidelines for CPR are updated every five years, researchers agree that the technical aspects of CPR may be simple but performing them correctly has proven difficult (Hunt et al., 2009; Hopkins, 2006). The latest recommendations by the AHA (2010) suggest that the most important component of CPR is getting the heart restarted by providing aggressive compressions in a timely fashion. In past research, nurses as first responders showed delays and lapses in emergency care as they prepared the patient's room for the critical care team instead of providing basic CPR (Hopkins, 2006). Also, a study with hospital mock code drills revealed at least one resuscitation and miscommunication error occurred at each drill, suggesting that resuscitation errors occur frequently (Hunt et al., 2007).

\section{Resuscitation Errors}

In a prospective study, researchers' suggest that chest compression rates during cardiopulmonary resuscitation are suboptimal and there's no easy method to evaluate the technical aspects of CPR, while in progress, in order to make improvements (Abella, Sandbo, Vassilatos, Alvarado, and O'Hearn, 2004). This prospective study evaluated three hospitals compression rates from April 2002 to October 2003 to determine a correlation between inhospital cardiac arrest rates with published resuscitation recommendations. 
In 2004, a group of research physicians observed 97 cardiac arrests in a 600-bed academic medical center, a 600 bed referral hospital, and a 400-bed community hospital to determine appropriate compression rates using a personal digital assistant (PDA) designed to record such events (Abella et al., 2004). The PDA was designed to record the start and end times of each event in real-time, and the total number of chest compressions given during each arrest (Abella et al., 2004).

In this study the mean age of the patients was 62 years, and $34 \%$ were women. The racial distribution was $38 \%$ black, $35 \%$ white, $24 \%$ other, and $2 \%$ unknown race (Abella et al., 2004). Fifty three percent of the cardiac arrest events took place in intensive care settings and $45 \%$ in the general ward. Two cardiac arrests were recorded in other locations (1 in radiology and 1 in cardiac catheterization laboratory) (Abella et al., 2004).

The frequencies of presenting cardiac rhythms were as follows: $15 \%$ ventricular fibrillation/ventricular tachycardia (VF/VT), 60\% pulseless electrical activity (PEA), 10\% asystole, and $15 \%$ other (indeterminate rhythms) (Abella et al., 2004). The return of spontaneous circulation occurred in $40 \%$ of patients. However, chest compression rates were less than $90 / \mathrm{min}$ during the first 5 minutes of resuscitation $28 \%$ of the time and less than $80 / \mathrm{min} 13 \%$ of the time (Abella et al., 2004).

Study results revealed that chest compression depths were too shallow and ventilation rates were high, and performed at a rate of more than 20/min well above the AHA recommended rate. This research study suggests that CPR quality may be highly variable in actual practice resulting in unpredictable physiologic and survival outcomes. This study also supports most published clinical studies involving cardiac arrest outcomes, where the quality of cardiopulmonary resuscitation remains unmeasured because of many confounding variables 
(witnessed or un-witnessed arrest; availability of necessary equipment i.e. resuscitation mask; lay person vs. medical professional performance and training) (Abella et al., 2004).

The study results did not link specific parameters such as chest compression rates or timing of the compression with any contribution to survival rates since the compression rate of less than $100 /$ min did not follow the AHA recommendations. This study suggests that these study findings may not be generalized to the entire population due to deficiencies related to human factors in CPR performance (Abella et al., 2004). Further research and observations of CPR performance are needed to ascertain the frequency of errors during resuscitation in order to make improvements.

\section{Quality of $C P R$}

In 2008 several researchers suggested that the quality of CPR during advance cardiopulmonary life support (ACLS) training remains sub-optimal after evaluating the quality of CPR during a six month period (Perkins, Boyle, Bridgestock, Davies, Oliver, Bradburn, Green, Davies, and Cooke 2008). Ninety four doctors and nurses working in critical care/emergency areas and who routinely participate on resuscitation teams were evaluated as part of this observational study to determine the number of compressions, no-flow time, and the duration of pre-shock pauses as well as the overall quality of CPR. Forty six individuals participated in the study before the implementation of the 2005 AHA recommended guidelines, and 48 individuals participated in the study after the new guidelines were implemented (Perkins et al., 2008).

The training exercises consisted of 4-6 participants in each group using a variety of CPR sessions (Ventricular fibrillation, ventricular tachycardia, asystole and pulseless electrical activity) with a Resusci-Annie mannequin. In each session, one participant was chosen to be the 
team leader and had the responsibility to delegate tasks. All participants had previous experience in performing CPR in the clinical setting. The other team members were instructed to support the team leader. Course instructors provided constructive feedback after each session regarding the participant's performance on technical skills and task delegations. The course also provided an opportunity for the participants to improve their CPR skills and work as an efficient team.

The results of the study showed that there were significant delays in initiating chest compressions following cardiac arrest in both groups (2.1 minute vs. 1.6 minute). The average compression depth was sub-optimal (too shallow), and in $77 \%$ of the cases the mannequin was intubated. The study results only showed slight improvements in skill performance over sequential simulations but they were still suboptimal using criteria from either the 2000 or 2005 CPR AHA guidelines (Perkins et al., 2008). CPR skill performance measures remain suboptimal because training courses are too short and infrequent as participants are not fully engaged (vested) in the exercise. These individuals may possibly assume that they have sufficient information about CPR to perform the task correctly and have no further need for CPR training. Also, frequent changes (every 5 years) made by the AHA in the recommended guidelines for CPR may provide an additional layer of confusion since recertification is required every two years.

Perkins and colleagues also suggested that after twenty five years of training individuals in CPR, the retention of cardiopulmonary skills are closely associated with the instructor, learner, curriculum, and frequency of timing in which the training takes place (i.e. once per year or biannually) (Perkins et al, 2008).This suggests that the simulated environment may be the future modality for acquiring and maintaining skills in various resuscitation venues such as adult 
advanced cardiac life support (ACLS), pediatric advanced life support (PALS), and neonatal advanced life support (NALS).

The Joint Commission on Accreditation of Healthcare Organizations (JCAHO) has also recognized the importance of improving individual's willingness to initiate effective CPR and suggest the need for such skills to be perfected in non-healthcare industries as well (JCAHO, 2008). Researchers from these studies suggest that continued investigations are needed to improve CPR performance, and simulation may provide a safer and less stressful environment to uncover or correct deficiencies (AHRQ, 2010). Other interventions such as color coding medication devices to support safe medication administration strategies in conjunction with simulation may provide a second layer of safety.

\section{Interventions used to Identify and Reduce Errors}

A major concern for hospitals and other healthcare organizations is to improve safety at the point of care. In the last decade, it has been very difficult to create a balance between high quality care and safety due to the complexity of healthcare systems. Most interventions have been met with marginal improvements in an effort to decrease the 1.5 million medication errors that occur each year. A color- coded system used in both pediatrics and anesthesiology has made some progress in the reduction of pharmaceutical mistakes when added to medication administration strategies. Using a color-coded approach in various high risk areas in healthcare have been known to provide order in mass casualty situations and medication labeling. This approach has also been used as a tool to provide faster and safer selections of pediatric body size-bases (examination tables) for computerized tomography (CT). 


\section{Color Coding}

Many different systems have been developed to establish order during casualty situations or in disasters. These systems have been devised using various acronyms such as start; simple triage, and rapid treatment. Other advanced systems during mass casualty situations use colors to assign categories (i.e. red tags needs immediate attention, yellow tags require observation only, green tags are reserved for the walking wounded and these patients may have to wait, white tags are used for minor injuries, and black tags are designated for the deceased) (Stoppler, 2007).

Anesthesiologists have also used color-coded labels for high-risk medications in syringes, and in recent studies they've used colors to distinguish between intravenous lines as a way to reduce dangers in mixing potent drugs (Porat, Bitan, Shefi, Donchin, and Rozenbaum, 2009). In a recent study by Porat et al (2009), color-coded labels were used to identify task completion of medication administration among a group of 61 critical care nurses using two mannequins in an intensive care bed (control bed and intervention bed). These nurses had an average of 2.5 years of ICU experience, and needed to complete six task sets. Although demographic data were collected, the sample was not further described using demographic data, and the analysis suggests that age was the only demographic variable found to be significant.

The six task sets included the length of time it took these nurses to identify the correct syringe, label an IV bag, locate the syringe pump, identify a peripheral vein, describe all drugs and lines, and identify an error in the treatment setting. The results from this study revealed that the average performance time for all tasks, labeling bags, description of drugs and lines, and error identification at the bedside were statistically significant $(\mathrm{p}<.005$ or $\mathrm{p}<0.001)$ when the control bed was compared to the intervention bed (Porat et al., 2009). These results indicated a clear advantage of the new color-coded labeling method at the intervention bed when compared 
to the current method at the control bed. Although this study was performed to develop a new labeling method to improve patient safety and medical staff efficiency, it also demonstrated that simulation technology and color coding strategies can be an effective combination used to improve training.

In a randomized controlled study, researchers compared the utility of a standard pediatric resuscitation cart with a pediatric resuscitation cart based on the Broselow tape (Agarwal, Swanson, Murphy, Yaeger, Sharek, and Halamek, 2005). This study was performed to determine which resuscitation cart allowed for faster access to equipment, an accurate selection of the appropriate size equipment, and better satisfaction among users. Twenty one physicians and nurses who were PALS certified took part in two successive simulated pediatric resuscitation scenarios; an eight year old with septic/hypovolemic shock and a one year old with status epilepticus (Agarwal, 2005). The order of the scenarios and the resuscitation carts were randomized to generate four potential pairs (standard cart/8- year old, Broselow cart/8-year old, standard cart/1-year old, and Broselow cart/1-year old).

The resuscitation leader was scripted to ask participants for specific equipment/items from the carts in a particular order. The time it took the participants to retrieve the requested equipment/items were collected. Also, a post scenario survey was given to ascertain the ease of utilizing the resuscitation carts using a 5-point Likert type scale (1-not at all easy to 5 very easy to use) (Agarwal, 2005). The Broselow resuscitation cart had color-coded drawers corresponding to particular length/weight ranges on the Broselow pediatric emergency tape, and the equipment was organized by patient size. Also, four modules were placed within each drawer containing intravenous, intraosseous, intubation, and oxygen delivery supplies in a labeled 
Ziploc bag. All participants were familiar with the standard cart from prior experience with real pediatric resuscitation events.

The results of Agarwal et al (2005) randomized study revealed that participants needed significantly more time to retrieve items from the standard resuscitation cart when compared to the Broselow resuscitation cart. The researchers' suggested that the features of the color-coded system using the Broselow pediatric resuscitation cart allowed participants to retrieve the appropriate equipment quickly and easily (99\% vs. 83\%), (Agarwal, 2005). According to the survey results, two thirds of the participants preferred using the Broselow resuscitation cart over the standard cart (Agarwal, 2005).

The use of color-coded organized equipment during resuscitation events may enhance provider confidence and increase patient safety measures. Using a well organized system during emergencies helps eliminate unwarranted mistakes and saves precious time needed for survival. Although the number of participants in this study was small the idea of organizing an emergency system using a color-coded method can theoretically reduce chaos and improve functionality in any environment. Further research is needed to determine if color coded systems can improve patient safety in a variety of settings (especially during emergencies), and simulation can provide a safe environment to practice emergency measures.

\section{Simulation}

Simulation appears to be a promising intervention that has the potential to increase patient safety, decrease medical errors, improve team training, and establish the foundation of a culture of safety in healthcare. Simulation may also help researchers understand the effects of behavior and attitudes towards simulation as educators continue to focus on other patient safety measures. A plethora of simulation initiatives have been aimed at increasing the capacity of 
simulation by training additional faculty/instructors in an effort to increase access to simulation, and ensure affordable simulation resources (FCN, 2010). However, many barriers to expanding simulation as an intervention to prevent errors still exist because extensive resources are required (i.e. space, equipment, and qualified personnel) to provide a holistic experience that mimics a true emergency situation.

The ability to measure appropriate psychosocial attributes of technical skills of health care professionals has been difficult without using a measurable objective test; such as the national board or specialty certification examinations. The use of simulation brings a host of sophisticated devices to teach and provide objective evaluations of the trainee's technical abilities without risk to patients (Issenberg and Scalese, 2007). These innovative state-of-the-art simulation devices used to teach basic skills and complex technical maneuvers through repetitive proctored challenges, will enable the detection of medical errors and near miss incidents while individuals practice in a safe environment (Issenberg and Scalese, 2007).

Simulation technology may be used to identify various medical errors during emergencies, guide mock code training exercises, and evaluate patient safety measures that improve team training. The current use of simulation technology helps instructors discover the effects of behavior and attitudes towards simulation while focusing on other patient safety measures where there's a high rate of medical errors (Issenberg \& Scalese, 2007).

\section{Evolution of Simulation}

The uses of simulation to train healthcare professionals are becoming a standard.

However, nurses have been simulating disease processes, enhancing situational awareness, and providing procedural training since the inception of formal nurse education (Jeffries, 2004). In this formal setting simulation training has been used to teach psychomotor skills and physical 
assessments techniques among students using inanimate objects, classmates, and task trainers (Jeffries, 2004). Various task trainers allow students to practice an unlimited amount of procedural techniques until competence have been met. Today, simulation has been combined with computer technology to generate new sophisticated models that enhance experiential learning and reduce learner anxiety (Jeffries, 2009).

In 1966, Drs. Abrahamson, Denson, and Wolf created a more advanced patient simulator (SimOne) that mimicked true physiologic conditions and responded to anesthetic agents. Around the same time, Dr. Michael Gordon and colleagues were building a cardiac simulator (Harvey) to teach physicians how to recognize a variety of cardiac arrhythmias (Gordon et al, 1969). However, SimOne and Harvey simulators were only used in physician training programs, and never generated use from a wider audience until 20 years later when these highly sophisticated simulators became commercially available (Cooper and Taqueti, 2004).

In 1990, the development and refining of an adult size computer based physiologically functioning device (mannequin simulators) that mimics a disease state or a malfunctioning organ opened the door for medical simulation programs around the world (Weller, Bloch, Young, Maze, Oyesola, Wyner, and Dob et al., 2003). Most model driven simulation programs use computer-based software to guide training exercises in building new curricula or adding components that will enhance existing programs. In healthcare, many simulation programs consist of life-size robot type mannequins also known as (human patient simulators -HPS) which are typically used for both high and low fidelity based scenarios along with standardized patients.

High fidelity simulators or scenarios are defined based upon the level of intensity needed to complete various invasive procedures in a given scenario. Low fidelity scenarios are most 
commonly done with standardized patients (humans) and do not include invasive procedures. High fidelity simulation training may use both static mannequins like resusci-Anne and sophisticated computer-based HPS to provide adequate models for invasive maneuvers. The HPS simulators have life like features including: exact weight and height of an adult size male or female, realistic facial features and limbs, and practical physiologic components (blinking eyes, chest rising and falling, sweating, cyanosis, and palpable pulses) (Gordon, 2005).

High fidelity simulators are used in more complex scenarios for training in acute emergencies where hands-on experience is paramount in stabilizing the patient (Gordon, 2005). Advance life support training, medical emergency, and acute situational awareness as well as team communication are only a few training objectives instructors choose when using high fidelity simulators. However, creating simulation programs can be both frustrating and expensive, but establishing a successful program must include the basic elements of simulation training: creating realistic scenarios, allowing debriefing, creating objectives with measurable outcomes, and using validated tools to ascertain reliable results (Gaba, 2004: Issenberg and Scalese 2007).

\section{Current Use of Simulation}

The multifaceted use of computers has propelled the world into a new era of total reliance on advance technology. Computer generated education and training programs in healthcare has put simulation at the forefront of patient safety and health care training (Gordon, Tancredi, Binder, Wilkerson, and Shaffer, 2003). Simulation allows the learner to make mistakes during training exercises of healthcare's most critical care systems using computer technology to improve performance. Hence, simulator manufacturers are finding it difficult to keep up with the demand for equipment as advances in medical simulation continue to seek technologies that will 
improve patient safety, reduce medical errors, ensure provider competency, increase emergency response time, and reduce health care costs. Continued advances in computer technology are needed to produce more realistic and higher quality simulators to help participants suspend disbelief (Weller et al., 2003).

In 2005, Issenberg and colleagues described a standardized patient as a patient encounter that is consistent in content of verbal and behavioral responses when using a HPS or a trained actor to elicit objective oriented responses from students. Thus, most simulation programs use standardized patient encounters as a routine tool to examine assessment skills (Issenberg et al., 2005). Issenberg and colleagues (2005) also suggest that the interest in simulation technology as a strategy to teach psychomotor and critical thinking skills has increased in the last two decades since marked improvements (increased mobility, improved physiologic parameters, and simple programming to provide easier use) were made in computerized mannequin simulators. These high tech simulators play a pivotal role in medical education to help improve the performance of multidisciplinary resuscitation teams (Issenberg and Scalese, 2007).

\section{Simulation Effects on Behavior and Attitude}

In a prospective study, a human patient simulator (HPS) was used to determine the effect of simulation on medical emergency team training behavior and attitude (Wallen, Meurling, Hednman, Hedegard, and Tsai, 2007). Fifteen medical students (7 males, 8 females) participated in a one week simulation training course that consisted of two didactic lectures over a two hour period in trauma care. Eight trauma scenarios were presented to allow students to use a team approach. Four of the five days were used for orientation to the simulation setting and three days were allowed for practice. Only five of the eight scenarios were presented on the actual study day excluding the pre practice and post exams. The types of scenarios were not clearly described 
but were selected on the basis that they included common injuries that required urgent attention. The scenarios also required the participation of multiple medical professionals because the medical students did not have enough knowledge to manage the patient alone. However, the patient's chief complaint and the symptoms were made easy to recognize.

The participants' attitudes were measured using a modified version of the operating room resource management survey (OTRMS). Behavior performance was obtained from video analyses post session using the global rating scale (GRS) (Wallen et al., 2007). Ten items on the GRS scale were used for rating crises management behaviors, six items measured team attitudes, and one item measured the overall team performance of leadership skills. The GRS scale includes 10 behavioral items anchored for rating on a 5-point scale ranging from 1 (not acceptable) to 5 (excellent), and the $11^{\text {th }}$ item measures overall team leadership. The OTRMS scale used a five point Likert type rating, with choices ranging from 'disagree strongly' to 'agree strongly'.

The study results show that in $93 \%$ of the group participants perceived the simulation exercise to be realistic (setting and equipment). Only one item on the OTRMS questionnaire showed a significant change between pre and post test scores and suggest there's no situation in which a junior member of the team should assume control of patient management $(\mathrm{p}=0.025)$ (Wallen et al., 2007). In all 15 groups (included two passive trainers as members), three key team skills (assumption of roles, communication with other team members, and recognition of limitations/call for help) were demonstrated. Also, six team component behaviors (knowledge of the environment, anticipation of planning for potential problems, distribution of workload, delegation of responsibility, attention to allocation, utilization of resources, and professional 
behavior/interpersonal skills) improved in response to the course (both didactic and skills training) among the groups (Wallen et al., 2007).

The study results did not show any improvement in attitudes toward safe teamwork and suggest that it may have been related to the minimum time (20 minutes) allocated to team work concepts (Wallen et al., 2007). Based upon these study results, attitude is a less reliable marker of training than behavioral change. Future studies using a greater amount of time in the simulated environment are needed to ascertain more concrete differences between group training using simulation only or as a combination with didactic lectures. Future research in simulation training must focus on all aspects of the team to enforce that all the roles of the leaders and followers are equally important. Although simulation training is laborious and requires additional resources compared to standard skills and team training, it may be worth the sacrifice, if it reduces medical errors, improves overall team performance and attitudes towards simulation based education (Wallen et al, 2007). Simulation-based training courses are designed to help narrow the gap between expected and actual performance of in-patient resuscitation teams by reducing barriers to simulation.

\section{Barriers to Simulation}

Barriers to nurses' participation in simulation training as well as nurses' priorities for simulation courses are influenced by prior simulation experience, professional experience, and the hospital setting (DeCarlo, Collingridge, Grant and Ventre, 2008). To help identify barriers to nurses' participation in simulation, 523 surveys were evaluated from full-time and part-time nurses in an academic children's hospital (DeCarlo et al., 2008).

In this study, researchers evaluated three barriers to determine whether prior simulation exposure, professional experience, and practice location had an influence on participants' 
priorities for simulation based education. Data were collected using a 54 item survey with a 5point Likert type scale, a checklist of yes /no answers, and multiple choice questions. The survey used in this study was designed to collect data on nurses' professional demographics (e.g. level of education, years of experience, area of hospital practice, and professional responsibilities), history of simulation experience, perceived barriers that would prevent or hinder participation in simulation-based training, and suggested priorities for simulation based courses.

The primary outcome of this study was to determine the relationship between barriers that prevented participants from pursuing simulator-based training and nurses' perception of simulation (DeCarlo et al., 2008). The study results revealed that nurses with prior simulation experience selected fewer barriers than nurses without simulation exposure (selecting yes to simulation is not real, $\mathrm{p}=0.02$ ) (DeCarlo et al., 2008). The study results also showed that years of experience were significantly related to barriers; "not the real thing" $(p=0.02)$, and unfamiliar with the equipment $(\mathrm{p}=0.01)$ as barriers (DeCarlo et al., 2008). Nurses selecting these two barriers had 5 or fewer years of work experience compared to those who did not select these items as barriers.

Additional findings also showed that areas of employment were significantly associated with "not the real thing" $(p=0.03)$ and a stressful/intimidating environment was an important barrier $(\mathrm{p}<0.01)$ (DeCarlo et al., 2008). Also, nurses' area of practice (acute care versus nonacute care) significantly influenced priorities for developing future simulation based courses ( $\mathrm{p}=$ 0.03), suggesting that nurses with acute care experience ranked the opportunity to manage rare events as a high priority than non-acute care nurses. This research study also found that prior simulation training is common among newly hired nurses practicing in a hospital setting, and these nurses seem to have an optimistic opinion about simulation benefits (DeCarlo et al., 2008). 
Unlike traditional lectures and other formats in which the learner is a passive observer, Issenberg and colleagues propose that simulation-based education is responsive to the adult student's need for an active learner-centered experience. This form of learning is embedded in an appropriate clinical context and allows for deliberate practice after a period of reflection. Other simulation experts state that simulation in healthcare is gaining widespread acceptance as a teaching tool which can be used to enhance any existing education curriculum (Seropian, 2003). The use of sophisticated mannequins with updated computer software, high-tech medical equipment, and highly trained personnel create a more realistic environment that help users' suspend disbelief (Seropian, 2003).

Experts in simulation training, recommend that simulation centers include education, research and development as well as develop curricula and techniques that can be validated to allow sharing of experiences across disciplines and institutions (Issenberg and Scalese, 2007; Seropian, 2003). Simulation is a powerful training tool and can be used to provide basic technical skills for various procedures or used as an evaluation tool. This type of learning promotes self-confidence, enhances technical skills, and provides a forum for team building (Seropian, 2003).

In Verplancke and colleagues (2008) observational study, simulation technology was used to evaluate the potential determinants of basic life support (BLS) quality and their association between demographic data and confidence scores in a group of nurses. Two hundred and ninety six nurses from non-critical areas were included in this study immediately following their annual mandatory recertification of BLS skills course (Verplancke, De Paepe, Calle, De Regge, Van Maele, and Monsieurs, 2008). All participants completed a questionnaire that requested demographic data on gender, age, wok experience, time since last BLS training, and 
time since last experience of CPR. Nurses were also asked to score their confidence in their ability to perform good quality BLS using a 4-point Likert (1 very weak, 2 weak, 3 good, and 4 very good) type scale to ascertain confidence scores in their ability to perform quality BLS.

Also following the completion of the questionnaire, participants were tested using a Skill reporter Resusci-Anne mannequin placed in a hospital bed and connected to a computer during a two minute BLS performance skills check. Seven variables were collected: number of ventilations/min, tidal volume, number of compressions/min, compression rate, and compression depth. In this cohort, $82 \%$ of nurses were female with a mean age of 37 years and had a mean of 14 years of work experience (Verplancke et al., 2008). Additionally, most nurses' last recertification course occurred more than 16 months prior to the study and 59 months prior to their last CPR experience. However, $50 \%$ of the nurses did not have any real life CPR experience. Forty nine percent of the study population scored their confidence level as one (very weak) while $48 \%$ scored their confidence at level two or three. Two nurses scored their confidence level at four (very good) (Verplancke et al., 2008).

The study results for actual CPR performance measures were low and correlated well with self-confident scores. Nurses with more confidence delivered more effective ventilations $(p=0.02)$. Male nurses' compression depths were deeper (correct depth) than female nurses, and they performed overall compressions significantly better $(p=0.001)$ than females. A shorter time since last BLS training was associated with a higher number of correct ventilations/compression ratios ( $\mathrm{P}=0.001)$ (Verplancke et al., 2008). These results were consistent with previous studies that show suboptimal performance of basic CPR skills among healthcare workers (Hunt et al., 2009). 
This study showed a significant difference in gender performance on compression rate and depth, suggesting that these differences may be due to the physical characteristics of body mass and muscle strength of the rescuer (Verplancke et al., 2008; Perkins et al., 2003). In summary, male gender, self-confidence, and recent BLS training were associated with good quality BLS. Therefore, authors of this study suggest that frequent BLS training using simulation technology may provide an opportunity to promote self-confidence and improve BLS quality (Verplancke et al., 2008).

\section{Team Training}

A research study using in situ simulation, a method of experiential learning to promote safety and team behavior among healthcare providers was conducted to identify the effectiveness of interdisciplinary teams during emergencies (Davis, Riley, Miller, and Hansen, 2008). This pilot study used four vital components (briefing, simulation, debriefing, and follow-up) to provide an overview of the effectiveness of interdisciplinary team training strategies on human factors (the provider, the team, the hospital system design for safety) and latent conditions (vigilance and surveillance in monitoring) during emergencies (Davis et al., 2008).

This study evaluated the effectiveness of the four components (briefing, scenarios, debriefing, and follow-up) of simulation training using 35 simulated obstetrics and neonatal emergencies, at six different hospitals involving physicians, nurses, and anesthesiologists. This qualitative study was conducted from January 2006 to January 2007 using three scenarios (e.g. placental abruption, ruptured uterus, and postpartum hemorrhage) based on real sentinel events that were created by an obstetrician, a clinical nurse specialist, and a research nurse specialist (Davis et al., 2008). 
The scenarios were developed with specific triggers (sudden clinical changes to divert the team's attention) and distracters (e.g. rude significant other, a language barrier, talkative mother, lack of a prenatal record, etc) to create stress for team members and to prompt specific human factor behaviors (i.e. leadership, shared mental model, situational awareness, and structured communication techniques of handoff to close the loop) (Davis et al., 2008). All sessions were videotaped and took on average 45 minutes to complete. The deliberate design of the scenario's features was to create stress and influence participants' to gain awareness of key communication and team learning behaviors.

In this pilot study post simulation surveys provided the team with an evaluation of the perinatal system processes and their individual performance which allowed for insight into the lapses in communication, team failures, and latent conditions (Davis et al., 2008). Other study findings (creating a failure mode and effect analysis, FMEA) gave direction to operational leaders on the unit level to continue with process improvement initiatives that include team training to promote staff development and education. The FMEA results showed that the top three failure modes were; 1) unclear role definition of team members during emergency Csection, 2) inconsistent process for ordering blood products in the operating room, and 3) lack of closed loop communication between the operating room and blood bank. These findings satisfied one of the joint commission requirements using root cause analysis to identify errors, and gave the administration team a framework for implementing additional safety measures to prevent patient harm.

Team work training in an in-situ environment may be an effective method of experiential learning that reinforces the values of becoming an expert team member. Simulation may be used to train healthcare personnel as a way to improve knowledge and clinical skills for a variety of 
technical procedures. Simulation may also be used to improve patient safety for both learners and faculty by reducing patient harm, learner anxiety, and improving team training. This study also provided a valuable communication forum for collective intelligence of hospital staff because members of a multidisciplinary team rarely train together.

In a crossover prospective study, researchers evaluated simulation based team work training for the emergency department staff to determine if simulation improved clinical team performance when added to an existing didactic curriculum (Shapiro, Morey, Small, Langford, Kaylor, Jagminas, and Suner, et al., 2004). The study followed one of four random teams of one attending physician, one resident physician, and three nurses, who were already trained in the Emergency Team Coordination Course (ETCC) in a 700 bed, level one trauma center. Each team was observed during a full eight hour shift (Shapiro et al., 2004).

The ETTC training included an eight hour didactic course taught by a physician and nurse pairs for mixed classes of 16 physicians, nurses, technicians, and other support personnel (Shapiro et al., 2004). The intervention group had a daylong session at the simulator center in addition to standard training. Also, three patient care scenarios (mild distress to cardiac arrest) were conducted with increasing complexity and lasted for 30 minutes followed by one hour of debriefing time. The Team Dimension Rating Form (TDRF) consisted of five, seven point items on a behaviorally anchored rating scale (BARS). The TDRF was used along with a seven item survey (5-point Likert type scale) to determine realism of the simulator and its impact on team performance (Shapiro et al., 2004).

Study results revealed that the median inter-rater reliability of the BARS scores were 0 . 67 across five dimensions and indicated a moderate agreement between the two raters (Shapiro et al., 2004). The mean TDRF scores between the intervention and control group showed no 
differences at baseline. The intervention group showed an improvement in team work behaviors ratings after the simulation training while the comparison group showed no change between the two observational periods (one hour and half duration). Thus, the study results showed that simulation training had a positive impact on team work behavior in a real clinical setting and the training may have the potential to improve patient outcomes (Shapiro et al., 2004).

Teamwork training for healthcare personnel may be supported by simulation technology that helps healthcare providers focus on team work behavior and not just technical skills. However, the study period of less than two months was very short and suggests that the recall of recently learned skills may not be beneficial as skills diminishes over time (Kozer et al., 2004: Shapiro et al., 2005). Also, this study did not address the Hawthorne affect, since the caregivers in the experimental group were not blinded to the intervention. The staff could have been cognizant of the study objectives (working well as a team) during the observation period and changed their behavior. Therefore, continued research and observations should be performed to identify areas for improvement in training and technical skills needed for resuscitation.

\section{Deficiencies in Team Training}

CPR interruptions have been shown to have negative consequences in overall survival and poor outcomes (Marsch et al., 2005). In a prospective observational study of 62 experienced practitioners (44 men), secondary activities during resuscitation were measured to identify unnecessary interruptions in CPR delivery (Tschan, Vetterli, Semmer, Hunziker, and Marsch, 2011). Twenty teams of general physicians treated a cardiac arrest patient in the simulator center at the medical intensive care unit. A confederate nurse (an actor covertly creating stress or obstacles germane to the scenario) briefed the physicians about the patient's condition and after two minutes the patient lost consciousness with the vital sign monitor showing pulseless 
ventricular tachycardia. The data were coded from videotaped recording using the 2005 AHA CPR guidelines. The resuscitation time evaluation began from the onset of ventricular tachycardia to the return of a normal sinus rhythm after the completion of one resuscitation cycle and the application of a counter shock (Tschan, 2011).

Coding secondary activities during unnecessary interruptions were categorized as: observing parameters displayed on the patient monitor; manually checking pulse or auscultating the patient with a stethoscope; technical handling of the defibrillator; and other activities (Tschan et al., 2011). The results showed that resuscitation exercises lasted between 2.8 and 7.6 minutes and the team engaged in unnecessary interruptions $32 \%$ of the resuscitation time. Additionally, one group member spent $84 \%$ of the time engaged in a secondary activity increasing unnecessary interruption time for the entire group. The majority of the unnecessary interruption time identified individuals focusing on the patient monitor or technical aspects of the defibrillator (Tschan et al., 2011). Also, all members of the group focused on the same secondary activity (technical aspects of the defibrillator) yielding $56 \%$ of unnecessary interruption time.

Overall this simulation study provided an observation of healthcare worker performance during CPR and described areas of the resuscitation process that resulted in unnecessary interruptions (Tschan et al., 2011). Although, these secondary activities may be considered necessary during the CPR process, they could ultimately derail or slow down the overall team performance resulting in poor survival outcomes. The authors' suggest that one intervention to reduce unnecessary interruptions is good task distribution at the beginning of the resuscitation process along with increase technical training to reduce potential rescuers preoccupation with secondary activities (Tschan et al., 2011). 
In accordance with this study, the Institute of Medicine has recommended the establishment of team training programs using simulation which may result in both superior resuscitation performance and improved survival outcomes (IOM, 2010). Simulation training programs can help individuals focus on improving organization, efficiency, and reliability of crises team responses as well as technical skills for ACLS/BLS. Therefore, simulation technology can be used as part of any curriculum to address, identify, and prevent medical errors for a variety of clinical or procedural skills (Gaba, 2004).

A study of an existing course (Medical Emergency Teams) that trains healthcare workers (nurses, respiratory therapists, and physicians) to respond to medical emergencies was completed using a human patient simulator to evaluate team performance (DeVita et al., 2005). This study consisted of three sessions running consecutively and each session ran for one hour and a half using the following four components: a power point introductory video, a brief didactic review of team performance concepts, simulated exercises (i.e. scenario; ventricular tachycardia induced dyspnea, acute myocardial infarction and arrhythmia, morphine overdose during patient controlled anesthesia, acute stroke with mental status change, and ventricular fibrillation), and facilitated moderated debriefings (DeVita et al., 2005). Also roles were predetermined (team leader, airway manager, airway assistant, procedure physician, chest compressions, medication/equipment cart, data manager/recorder, and bedside nurse) so participants could focus on treatment tasks, and all participants were ACLS certified within 2 years of their medical training.

Over a one year period DeVita and colleagues (2005) trained 138 individuals (physicians, nurses, and respiratory therapists) in their MET course to function as the medical emergency response team. Also, one specialist in hospital medicine and three intensive care specialists 
participated as team members, and no teams had more than eight people. The study results showed that the overall rate of simulated survival increased from zero percent to $90 \%$ across the three sessions that occurred each day. Most of the improvement in survival was observed between the first and second sessions (DeVita et al., 2005).

Additionally, the task completion rate improved from $31 \%$ to $89 \%$, and each simulated role (team leader, airway manager, airway assistant, procedure physician, chest compressions, medication/equipment cart, data manager/recorder, and bedside nurse) improved from $10 \%$ to $45 \%$ during the first session and $80 \%$ to $95 \%$ during the third session $(\mathrm{p}=0.002)$. Also, the post hoc analyses of the study findings revealed an improvement in the overall completion tasks between the first and second sessions $(\mathrm{p}=0.002)$ and between the second and third sessions $(\mathrm{p}=0.011)($ DeVita et al., 2005).

The study results also showed that a structured interactive, human simulator based team training curriculum can facilitate and improve simulated medical crises, allowing responders to assume predetermined roles and complete tasks associated with that role. However, if another responder possessed better skills for a certain role then a switch of that person into the role was appropriate (i.e. nurse bag mask providing ventilation or an attending physician providing bag mask ventilation prior to intubation). Therefore, the results of this study suggests that knowledge of what to do in a crises is not enough to ensure successful team performance, thus, implying that simulation training improves MET organization and performance (DeVita et al., 2005). However, the study population was not randomized and outcome survival was based on task completion of the ABC's of resuscitation and delivery of critical treatments in a timely fashion. These outcome variables may not reflect survival outcomes in a real clinical environment, and may prove challenging to replicate due to other risk factors associated with real emergencies. 
In another prospective study, researchers compared three simulation-based training methods for management of medical emergencies to determine whether full-scale simulation would provide better training than predominantly computer screen-based training (CSBT) (Owen, Mugford, Follows, and Plummer, 2006). In this study all participants $\mathrm{n}=61$ (resident physicians) had the same total teaching time that included an initial (pre-training) assessment by written tests (multiple choice questionnaire), self-assessment, and simulations of medical emergencies ('Ventricular tachycardia' and 'hypoglycemia') (Owen et al., 2006). All participants were tested 3 weeks after the last training (some were longer due to other commitments) using similar scenarios with tachycardia, hypoglycemia, and anaphylaxis post-training. The confidence of each trainee in performing a number of skills was assessed using a 16-item skills confidence questionnaire (SQC). Groups received computer screen- based training (CBST) using resusSim (an in-hospital program) (Owen et al., 2006). All technical skills were done using basic CPR trainers (Basic Buddy, Little Anne, and Resusci-Anne) with a number of cardiac arrest scenarios, using actual clinical equipment.

In this study, all three groups demonstrated improved knowledge of acute care of medical emergencies and relevant clinical skills and behavior (Owen et al., 2006). The multiple choice questionnaire of knowledge test (MCQ) scores improved significantly $(<0.001)$ following teaching in all three groups. There were no differences detected between groups. The study showed an overall improvement in self evaluated 'confidence' questionnaire (SCQ) scores ( $p<$ 0.001); however, the trainees were performing clinical duties throughout the study so improvement may not have been attributed solely to teaching received in the study. Results in this study also showed that group three had significantly higher scores $(p=0.047)$ in the VT-post 
scenario and performed significantly better than the other two groups (group one and two) in the anaphylaxis scenario $(\mathrm{p}=0.012)($ Owen et al., 2006).

Although these study results provide evidence that full-scale simulation training programs may improve scores when compared to either CSBT or low fidelity training (noninvasive patient assessments), these results may not be generalized to similar settings since all participants were able to gain additional knowledge during the training from other clinical sources (Owen et al., 2006). Also, the multiple choice questionnaire (MCQ) that tests knowledge and the skills confidence questionnaire (SQC) were not validated as part of this study to determine its use with similar audiences (Owen et al., 2006).

Simulation training may be a useful tool to train healthcare professionals for a variety of clinical situations when programs are created with clear objectives to attain measurable outcomes. Although, a large amount of data related to simulation education has been collected from schools and laboratory settings, more studies are needed to determine if such results can be translated into improved patient outcomes. Recently, more studies with simulation training have occurred in patient care areas defined as in-situ training (i.e. simulation of in hospital emergencies and CPR at the point of care: the first five minutes), and researchers are optimistic that these results can be extrapolated to explain or describe various patient outcomes (Owen et al., 2006).

The Ottawa Global Rating Scale (GRS) has been one of the first validated tools used in simulation to provide a scoring system for team performance. The GRS is a nine item tool that evaluates team performance in several crisis management resource domains. The GRS was recently used to evaluate a checklist (a modified version of the GRS) as a tool to assess performance in the resuscitation of critically ill patients during simulated emergencies (Kim, 
Neilipovitz, Cardinal, and Chiu, 2009). Fifty five first and third year medical students participated in two simulated scenarios in which the original Ottawa GRS was used to identify five crises resource management (CRM) skills based on recognized CRM literature (problem solving, situational awareness, leadership, resource utilization, and communication) (Kim et al., 2009). The overall rating for CRM performance was obtained using a checklist (a modified version of the GRS) and each category was measured using a seven-point anchored ordinal scale.

The two simulation session took place within a 2-3 week period. Each resident were able to lead at least one scenario and the order of scenarios were identical. The first scenario was a postoperative patient with cardiac arrhythmias, and the second scenario was a severe trauma patient in acute shock with hypoxemic respiratory failure. For each case the settings were the same and support staff provided assistance (trained actors played the role of nurse and respiratory therapists). All sessions were videotaped.

The study results revealed that there was a significant difference in the overall scores using the Ottawa GRS and CRM checklist for both sessions when comparing first and third year medical students. Inter-rater reliability for both the Ottawa GRS and CRM checklist was assessed using a calculation of intra-class correlation (ICC) scores of 0.59 and 0.61(Kim et al., 2009). These results suggested that the modified version of the GRS (checklist) was as good as the original GRS scale when comparing inter-rater reliabilities. Also, the cumulative checklist demonstrated an (ICC) score of 0.63 and 0.55 for the cardiac arrhythmias and trauma scenarios showed good reliability for these domains. Analysis of individual categories within the Ottawa GRS showed similar reliability scores for problem solving, leadership, and situational awareness (Kim et al., 2009). 
This study showed that the CRM checklist was favorably compared to the Ottawa GRS tool and may reliably be used to validate future simulation training courses using similar audiences (Kim et al., 2009). Tools to validate simulation activities are few because the field of simulation lacks an accepted gold standard for comparisons, making it difficult to find tools that will measure clinical outcomes. Currently, most studies with high-fidelity simulations use checklist categories to recognize best actions and behaviors for problems with recognized solutions (Kim et al., 2009).

Unfortunately emergencies in medicine do not have best solutions because of many uncontrollable variables such as; the type of emergency, age of the patient, availability of necessary equipment, adequately trained staff, appropriate staffing, and adequate time to respond to the emergency. Typically, emergencies create a challenging environment and make it increasingly difficult to measure healthcare provider's actions as well as performance of technical skills (Kim et al., 2009).

\section{Conclusion}

According to the Agency of Healthcare Research and Quality, medical errors are one of the leading causes of death in the US despite increasing concerns and raising public awareness of preventable medical errors (AHRQ, 2008). Developing medical error reduction strategies during emergencies has proven difficult due to many associated factors that promote an error prone environment. Some factors that prevent medical error reductions are; poor quality from professional practices, utilization of suboptimal healthcare products, medical procedures performed by least qualified clinicians, and disorganized healthcare systems that allow errors to occur (CAPSLink, 2003). Although, identifying and preventing medical errors during 
emergencies is difficult, simulation technology may prove to be one of the most promising strategies used to identify and correct errors during emergencies.

The challenge of creating less faulty healthcare systems or solutions to reduce medical errors has been met with much controversy as researchers develop simple and inexpensive ways to improve patient outcomes devoid of medical mistakes. Medication error prevention strategies using visible signage and checklists to reduce distractions and interruptions while preparing and administering medications have been shown to be one successful solution (Pape et al., 2004). However, this prevention strategy has not been widely embraced by the healthcare community resulting in limited research using visible signage and checklists to reduce interruptions while preparing medications. Many patient safety strategies do not significantly reduce the approximately 1.3 million medication errors that occur in US hospitals and other medical facilities each year (Pape et al., 2004; Lipshutz eta al 2008). Visible signage and checklists are good medical error prevention strategies and more research is needed to determine the use of these interventions during emergencies where chaos, interruptions, and medical errors occur more frequently.

Previous research studies have demonstrated that medical errors during emergencies (codes) are 39 times more likely to result in harm and these errors occurred more frequently than non-code related errors (Abella et al., 2004; Lipshutz et al., 2008). Major errors found in one mock code (staged cardiac arrest) study were: a failure to start chest compressions immediately, the lack of ability to recognize and treat common arrhythmias, and an overall poor adherence to the AHA recommendations for all emergency measures (ventilation, compressions, medication administration, and defibrillation) (Hunt et al., 2007; Hunt et al., 2009). 
Studies also have shown that low survival rates of cardiac arrest victims regardless of the setting (in or out of hospital) were associated with poor CPR performance by first responders. Medical professionals as first responders were distracted or interrupted with other resuscitation tasks (preparing medications, setting up the defibrillator, preparing the necessary equipment needed for intubation, etc) which may have contributed to their poor CPR performance, and low patient survival rate (Valenzuela et al., 2005: Aufderheide et al., 2004).

Although CPR performance skills are mandatory for recertification of all healthcare providers, survival after cardiac arrest continues to have dismal outcomes. Studies associated with low patient survival were linked to poor performance of cardiac measures (ventilation, compressions, medication administration, and defibrillation) by healthcare providers due to; knowledge deficits, skill retention, poor team communication, and unintentional deviations of the American Heart Association recommendations for CPR performance.

In an observational study by Aufderheide and colleagues (2004), paramedics routinely hyperventilated individuals in (out of hospital) cardiac arrest by more than doubling ( 30 breaths/min) the amount of ventilations (12 breaths $/ \mathrm{min}$ ) recommended by the AHA. This study also showed that paramedics were interrupted and preoccupied with other resuscitation task s (getting the defibrillator ready, starting IV`s or preparing medications) resulting in poor performance of continuous compressions as recommended by the AHA.

Although, no patients survived in the Aufderheide and colleagues (2004) observational study of out of hospital cardiac arrest, resuscitation performance measures for patients enrolled after retraining paramedics followed more closely the AHA recommendations. These studies suggest that deviations from recommended guidelines are common (Aufderheide et al., 2004). 
The study design was revamped as the principle investigator stopped data collection to retrain paramedics to follow the American Heart Association for CPR performance measures.

Although CPR may seem to be a simple procedure that holds great benefit to any patient in cardiac arrest, the process of performing CPR correctly has been difficult to achieve and measure. However, the use of simulation technology to re-create emergency situations such as cardiac arrest may allow investigators to identify and correct poor CPR performance measures without patient harm.

Using simulation in conjunction with other patient safety measures such as color-coding methods may further reduce user error and improve patient outcomes. Color coding methods allow easier and safer use of complex systems by providing a simple organized format. The use of a color coding system allowed radiology technicians to select the correct size tray/beds for pediatric patients to perform safe CT scans. Pediatric medication errors are usually fatal and occur due to the complex system used to determine medication doses. The difficulty in creating a standard system for medication dosing in children is due to the various sizes and weights of this population. Dr's Broselow and Lueten tackled this challenge by developing a color coded system to simplify medication administration in pediatrics during emergencies.

A simple color-coded tape measure was designed using length as a correlation for approximate weight in pediatric patients. Dr. James Broselow an emergency room pediatric physician discovered that a child's length/height directly correlated to his/her ideal body weight, and this measurement method could be used as a quick reference when administering medication during emergencies. The use of the Broselow tape has been accepted nationwide as a standard tool for emergencies among pediatric patients. The success of the color-coded tape measure in pediatric emergencies has lead to additional standardization of pediatric critical measures. Now 
many hospitals use pediatric crash carts that have been organized based upon the Broselow tape in which each drawer contains medication and equipment for specific size children using distinct colors.

In an effort to improve care, color-coded methods combined with simulation can be combined to provide maximum safety measures. Simulation provides opportunities for participants to learn from the experience and make mistakes without increasing risk to patients while providing assurance that each student receives the same learning experience for a given clinical scenario (Issenberg and Scalese, 2007). Simulation also reduces the variability in the teaching/learning process by allowing each participant to gain the same clinical experience regardless of the available resources to manage high-risk conditions (emergencies).

Several studies have shown that students, who participate in simulated training sessions, attain desired knowledge, demonstrate self-reported competence in skills, and gain increased self-reported confidence in abilities to perform specific skills or in a explicit situation (Gaba, 2004; Issenberg and Scalese, 2007). Although there are no large scale studies that show compelling evidence to support the use of simulation as an alternative to clinical practice, simulation should be viewed as a tool to supplement traditional training. Nevertheless, continued research is needed to assess the transfer of knowledge and skills from the simulated environment to the clinical setting, to predict the value of learning in the actual clinical setting and during emergencies.

In summary, simulation has shown promise in identifying medical errors during emergencies and helped create interventions to reduce them. Simulation has also been used as an ideal tool to recreate emergency situations in an effort to prevent errors that occur 39 times more frequently than errors during routine care. This tool helped researchers review actual CPR 
performance measures of healthcare providers for both adult and pediatric patients. Although animal research had been used to understand the physiology of CPR, simulation can be used to understand the technical aspects of CPR performance skills. This understanding can help researchers and instructors improve the quality of CPR by providing additional classes, enhancing CPR techniques, and allowing CPR performance measures to evolve until technical skills are perfected.

The current use of simulation has also been important in providing venues to promote team training as well as identify deficiencies in team work skills (communication, task completion, and collaboration). Unfortunately, none of these interventions will have an effect on reducing medical errors if healthcare providers do not have positive attitudes in embracing this new form of experiential learning. Changing behaviors among healthcare providers has always been a challenge as new technologies merge and old methodologies are replaced. However, simulation technology may prove to be one of the most promising strategies used to identify and correct $\mathrm{CPR}$ performance measures during emergencies as healthcare providers began to embrace this method of training. 


\section{Chapter III}

\section{Methodology}

\section{Research Design}

This study used a quasi-experimental design to test the use of a smock system among two groups of nurses during CPR training in a simulated environment. Each group intervention and control (20 groups of 8 ) consisted of 320 nurses participating in different roles on a resuscitation team. Nurses in the control group (160) completed a clinical scenario requiring resuscitation measures using the current standard of training for basic life support (BLS) or advance life support (ACLS) in a hospital setting. Nurses in the intervention group (160) used all the same measures as standard training (control group) in addition to wearing color-labeled smocks that identify the resuscitation role for each individual participating in the training exercise. The study evaluated the nurses' task performance skills for ventilation, chest compressions, medication administration, and defibrillation along with team performance and the total number of medical errors committed during the exercise. Data were collected by observation in real time to provide immediate debriefing, and video recordings were reviewed post session to obtain data for the study. Observation data was not used as part of the analysis.

\section{Sample}

A convenience sample of 320 nurses (40 groups of 8 nurses) employed at the University of Miami-Jackson Memorial hospital was recruited for the study. Inclusion criteria: any age, race, working in any specialty area within the hospital, having less than 20 years of clinical experience (not likely to have worked in a critical care area) and having recently (within recertification window-two years) taken a CPR refresher course as part of their annual mandatory training. Exclusion criteria: nurses with severe handicaps that limit their physical 
ability to perform effective CPR (i.e. deafness, severe carpal tunnel disease, back problems, already taking prescribed medication for anxiety or other mental illnesses exacerbated by stress, etc).

All nurse participants were allowed to participate in the study, either, coming to the hospital on their day off, or during working hours if the time was approved and set aside for uninterrupted CPR training. A power analysis for sample size was done using G-power 3.1 software. A large effect size of 0.8 for one-tailed two-sample t-tests with alpha 0.05 and $80 \%$ power yielded a sample size of 40 groups, 20 control and 20 intervention groups.

\section{Setting}

The study was conducted in the University of Miami-Jackson Memorial Hospital Center for Patient Safety simulation laboratory and two other training facilities within the hospital. Jackson Memorial Hospital (JMH) is a tertiary teaching hospital with more than 1,500 licensed beds serving all of Miami-Dade County, and provides specialty services to the Caribbean, South America, and Puerto Rico. JMH is also a magnet for medical research, a referral center, the only adult and pediatric level I trauma center in Miami-Dade County, and a clinical training site for local nursing school's LPN, ASN, BSN, MSN, and Doctorate programs. The University of Miami and Jackson Memorial Hospital employ more than 50,000 nurses, physicians, and other healthcare workers.

The Center for Patient Safety (CPS) is a joint venture with the University of MiamiMiller School of Medicine, Jackson Memorial Hospital, and the University of Miami, Department of Anesthesiology. The 8,000 square feet of space allocated to the Center for Patient Safety resides in Jackson Memorial Hospital and it hosts a variety of learning venues including: didactic training, computer based learning, procedural training, and simulation training. In CPS, 
over 2,000 square feet of space is dedicated to simulation-based training activities and research. The Center houses a variety of adult and pediatric high-fidelity mannequins along with four complete replicas of patient care areas (an emergency room, an operating room, a patient care room, and an outpatient clinic). All rooms are equipped with video and audio recording capabilities to provide optimal real time feedback during training.

All simulation rooms have fixed cameras with remote controlled lenses, microphones, and speakers mounted in the ceiling; and information from these devices is fed to a DD recorder in the control room. The DVD's are formatted and played back to the participants in a conference room and projected on a large screen for review/debriefing.

\section{Procedures}

Following IRB approval at FIU and the clinical site (UM-JMH), nurses attending training courses were approached at the beginning of the class to explain the study, determine their interest in participating, and to obtain informed consent. The first 160 nurses were included in the standard care group and the next 160 nurses were included in the intervention (smock) group. The purpose of separating the groups and enrolling all of the participants in the control group first was to prevent a carryover effect regarding the nurses becoming aware of the SMOCKS through rumors. Each group of 160 nurses was divided into smaller working CPR groups of 8.

These smaller groups allowed participants to respond to a clinical scenario using eight possible roles commonly seen in a hospital code blue situation (team leader, person providing ventilation, person providing compression, defibrillator operator, medication administrator,

recorder, IV nurse, Circulating nurse). CPR skills performance on the four primary measures (ventilation, compression, medication administration, and defibrillation) along with team performance and medical errors was evaluated in both groups. 


\section{Control and Intervention Groups}

Each group of nurses was given an orientation prior to the start of the study once the consent forms were signed. This orientation included a small introduction about the study, detailed instructions about the mannequins (the basic functions and limitations of the mannequin) and information about the room (cameras, microphone, telephone, monitors, etc.) The groups were given instructions to call a specific number for help and an operator (trained actor) assisted their call accordingly (i.e. calling a doctor, code team, rapid response team, laboratory etc). The group was also given information about the debriefing process which took place at the end of the scenario while reviewing the video simultaneously.

In each group of 8 nurses, one nurse (first responder) was given a clinical scenario (using a simulated mannequin complaining of pain or difficulty breathing). The nurse receiving the scenario started the case and after one minute the patient became unresponsive with no heart rate or respirations, and the scenario lasted a total of five minutes. The first responder was expected to call for help by initiating the rapid response team or activating a code blue emergency. The other 7 members of the group acted as the code team, assuming specific roles needed in real life code situations and proceeded as if they were called for a real emergency within the hospital.

The scenario lasted approximately 6 minutes (this included the first minute with the primary nurse) and was terminated whether the team completed all necessary tasks for adult resuscitation or not as described by the AHA 2010 guidelines. This time frame was adequate to allow for two - five cycles of compression (30/2 compressions to ventilations), placement of the defibrillator pads, and the administration of the first round of resuscitation medication if needed. This time frame also represented the most optimal time (4-6 minutes) for complete recovery with minimal to no brain damage. Additionally, if the team performed all of the correct measures of 
$\mathrm{CPR}$ in less than six minutes, then the patient regained consciousness and the scenario was terminated after six minutes of the session time.

For each session, participants were expected to perform CPR as if they would in a real life medical emergency situation using the correct equipment located on and in the crash cart (emergency cart). Participants were also expected to correctly use the life pack 20 defibrillator which is the standard external cardiac electrical device used to cardiovert a typical arrhythmia or pacing an extremely low heart rate at UM-JMH. The use of the defibrillator included putting the pads from the Automated External Defibrillator (AED) on the patient to create a perfect human machine interphase and provide the correct treatment for cardiac arrhythmias. The life pack 20 was used only as an AED for this study.

Each participant was also expected to administer the recommended medication for two of the primary drugs (epinephrine 1mg IVP and vasopressin 40u IVP). Participants were not expected to actually mix medications to make an IV solution but were required to assemble the single dose medication devices (prefilled syringe) to give an emergent dose of the correct medication. The team also announced what type of medications they were giving, the route to give the medication, and the amount to be given. Activities of all team members were manually recorded on the AHA technical assessment score sheet in real time by the instructor for immediate feedback during debriefing, and again by the research assistant from the video recording for the study.

A 12-15 minute debriefing session occurred immediately following each scenario to discuss the correct actions to be performed at each stage of CPR until complete recovery or death. During this time participants were allowed to ask questions and give comments regarding 
any aspects of CPR and on their individual or group performances. Participants were allowed to participate in only one scenario in an effort to identify the current CPR practices.

The training was conducted in the simulation center using the standard procedures and equipment routinely used for mock codes (teaching crash carts, life pack-20 defibrillator, wall mounted oxygen and suction delivery systems, and patient monitors). A Laerdal high fidelity (SimMan) Mannequin was used as the patient. The vital signs monitor was available to display continuous electrocardiogram and pulse-oximetry, along with a manual component for noninvasive blood pressure measurements. Initially the patient was breathing normally and the patient's nurse had to be able to detect respirations on the mannequin by looking at chest rise. Nurses were not allowed to participate in the study if they arrive more than 10 minutes late and/or if the main instructional components of the course were well underway.

\section{Intervention (Smock) Group}

Nurses in the intervention groups used all the same scenarios and procedures as the standard training (control group) as described above in addition to wearing color labeled smocks that identify the role for each individual nurse participating in the training exercise. There were eight different color labeled smocks representing the eight specific roles (team leader, ventilation, compressions, defibrillation, medication administration, IV nurse, circulating nurse, and recorder) needed for a complete resuscitation team. A brief instruction regarding the smocks and their usage was given during the introduction of the study for individuals in the intervention group. The smocks were placed in a plastic bag in clear view on the teaching emergency cart as part of the equipment needed to participate in the training. The intervention groups were instructed to wear them at all times when participating in the scenarios. 


\section{Scenarios}

Each group of 8 nurses (control and intervention) was exposed to one cardiopulmonary arrest scenario that required all members of the team to use skills and knowledge related to the main components of CPR (ventilation, compression, medication administration, and defibrillation). The scenarios were designed to simulate common medical conditions that resulted in sudden cardiopulmonary arrest within a hospital setting. The 4 different scenarios (same scenarios for control and intervention groups) consisted of a recent patient admitted from the emergency room and transferred to a single patient room on a medical-surgical floor. The scenarios consisted of patients exhibiting signs and symptoms of a cardiopulmonary arrest as a result of the following clinical conditions:

1. A 52 year old Hispanic male complaining of chest pain, shortness of breath, dizziness, and heart flutter and after one minute lose consciousness that result in cardiopulmonary arrest.

2. A 58 year old female diabetic patient admitted from the emergency room as a result of a severe hypoglycemic crisis and after one minute had a sudden cardiopulmonary arrest.

3. An 80 year old African American male complaining of chest pain, nausea, vomiting, shortness of breath, constipation, and limited physical activity with abnormal vital signs and after one minute he had a cardiopulmonary arrest.

4. A 45 year old African America female with an internal pace maker came for an emergency room visit because she was feeling faint, having shortness of breath, and sweaty with poor vital signs that result in a cardiopulmonary arrest after one minute. 


\section{Instruments}

CPR Scenario Technical Skills Assessment Score Sheet

The CPR Scenario Technical Skills Assessment Score Sheet is currently used in the $\mathrm{UMH} / \mathrm{JMH}$ CPR re/certification program and was used to assess the accurate completion (time and sequence) of each critical item related to CPR performance. This tool was used as part of the study and each item was scored using a three point Likert type scale $(2,1, \& 0)$; a score of two represents the task being completely performed; a score of one represents the task being partially performed; and a score of zero represents that no attempt was made to perform the task. A total group score was computed from each item listed on the assessment score sheet and the sum total of these items was recorded for the groups. The total means scores for each the control and intervention group were compared using the total scores divided by the total possible scores.

In this study two sets of CPR Scenario Technical Skills Assessment Score Sheets were completed; one by the course instructor in real time and the other by a research assistant post video review. The purpose of the second set of scores from the checklist was to assure that no areas were omitted by chance or completed by mistake (inter-rater reliability) and these scores were used for the study.

Time to implement compressions, ventilation, medication administration and defibrillation was measured by observation using the most current CPR Scenario Technical Skills Assessment Score Sheet which captures the technical skills from the AHA 2010 CPR guidelines. Start time for this measure began when the patient became asystolic (after first minute) or when the nurse assessed the level of consciousness (LOC) and ended 5 minutes after asystole. Time was compared to the AHA guidelines for each task in each of the four areas (ventilation, compression, medications, and defibrillation). If the task was completed by the time 
indicated on the AHA guidelines for that task, the team received a score of "1" and those who took longer received a score of "0" determined separately for each task. Also the actual time from LOC to the implementation of each of the four components of CPR (ventilation, compression, medications, and defibrillation) was obtained.

The time scores for each task were summed for each area (as subscales) and then scores for each area were summed to create a total score. Additional information on each group's emergency measures (frequency of ventilation, chest rise related to ventilation, rate, depth, and frequency of chest compression, medication administration, and defibrillation) was collected from video recordings. The total scores for each group were divided by the total number of items for that scenario to obtain a percentage. Since there were four scenarios, five groups of eight participants for both the intervention and control groups were exposed to one of four scenarios.

Medical error information and medication administration (omissions, wrong dosage, wrong route, and wrong medication) during CPR skill performance was measured from the video recording using the CPR Scenario Time and Medical Error Checklist with columns for time and errors. Number of errors in each area (ventilation, compression, medications, and defibrillation) was tallied, and the tallies were summed to obtain total number of errors for the scenario.

Medical errors beyond time and sequence were captured by counting the number/frequency of incorrect CPR procedures. These additional medical errors were considered as follows: any improper use of the bag mask ventilation device (i.e. wrong size mask, unable to obtain a seal, and no chest rise with each ventilation breath); giving medications not according to $\mathrm{CPR}$ recommendations (i.e. giving the wrong medication via the wrong route, omission of a dose, or any medication requested but not given); and applying compression at a depth or rate (incorrect hand placement) that was not consistent with the 2010 AHA guidelines. 
Further medical errors were considered when a misuse of the defibrillator occurred during CPR training (i.e. incorrect placement of pads or the inability to deliver a shock) when using the life pack-20 device. The total number of medical errors associated with each task was tallied for each group (20 controls and 20 interventions) from the CPR Scenario Time and Medical Error Checklist using information obtained from the video recordings.

Team performance was measured using the five-item Ottawa Abbreviated Simulator Session Crisis Management Skills Checklist (OASSCMSC) to assess five categories of crises resource management and team performance (problem solving, situational awareness, leadership, resource utilization, and communication). This checklist used a three point scoring system to indicate whether these team performance components were demonstrated during the scenario. Each task was assigned a numeric score from zero to two. A score of 2 was given for each item that was demonstrated fully, a one was assigned for partial completion of any item, and a score of zero was assigned if no items on the checklist were performed.

Summative scores were created for each of the 5 categories for each group (20 control and 20 intervention groups). A total mean score on the OASSCMSC was computed by adding the summative ratings for each category divided by the total possible score of the instrument. The OASSCMSC was validated with seven residents performing 32 procedures using a one way analysis of variance that showed scores increased with years of training and reliability was excellent with a Cronbach's alpha of 0.91 (Kim et al., 2009).

\section{Demographic Data}

Demographic data on nurse's age, clinical specialty, number of years employed in healthcare, previous simulation experience, last ACLS/BLS course, and an approximate number 
of times ever participating in a real life or mock emergency (code blue) was collected on a standard survey form.

\section{Data Collection}

All data collected during the scenario were recorded on the CPR Scenario Technical Skills Assessment Score Sheet by the ACLS/BLS instructor in real time during the simulated exercise for debriefing and by the research assistant during a review of the videos. A research assistant scored $100 \%$ of the sessions from video recordings of the CPR simulation exercises using the same checklists. The P.I. scored $25 \%$ of the session from video recording to determine the inter-rater reliability between the research assistant and the P.I. on the first two recordings for each scenario. Additionally, $25 \%$ of the data collected by the research assistant was reviewed by the P.I. or another research assistant to identify a drift in scores due to grader fatigue or boredom.

All data collected were described in aggregate form. Individual demographic data were de-identified by assigning each participant a unique study number as well as assigning a specific group number. Data on team performance and medical errors were collected separately post review of video recordings by the PI and the research assistant using the same process described above to prevent drift using the OASSCMS Checklist. In an effort to maintain study integrity and participant confidentiality, all videos will be destroyed five years after data analyses and last publication from the data set.

\section{Data Management}

The data from each participant are in individual files organized by a unique number and separated based upon the assigned group. Each paper file contains demographic data, a signed consent form, a CPR Scenario Technical Skills Assessment Score Sheet, and the OASSCMS checklist for group scores. A master log book and study files are kept in a locked office inside of 
a locked cabinet in the Center for Patient Safety with the participants ' names and their assigned study number. Only the PI, the PI's committee, and RAs have access to the data. Data was entered into a statistical program, SPSS for Windows.

The PI verified the data entry to minimize errors. A random sample of data was reviewed by the P.I. from data collection to data entry and analysis. Participants' records were reviewed for a signed consent form by participant and research team along with completed data forms for each training exercise. Additional safeguards were used to protect the study integrity which included double entry as a way to uncover discrepancies. The PI and study team used SPSS to examine frequencies and descriptive statistics to look for missing data and possible data entry errors. After correcting any errors, the PI merged the SPSS file of newly-entered data with the already-cleaned data. Data review was ongoing with continuous feedback from faculty as needed to maintain data quality.

\section{Data Analysis}

Data analysis compared tasks performance between the control and intervention (smock) groups on time to implement emergency patient care measures team performance, and medical errors. Comparing control and intervention (smock) groups during emergencies (codes) were to determine if there was a statistical difference in:

1. Time to implement compressions, ventilation, medication administration and defibrillation. Two sample t-tests were used to compare the intervention group (study smocks) on time-toimplement scores in each of the 4 areas and a total time-to-implement scores.

2. The number of recorded medical errors (i.e. sequence in initiating ventilation and compression; medication administration [giving the wrong medication via the wrong route,]; omission of ventilation, compressions, requested medications or defibrillation; or errors in 
providing ventilation, compressions, and or defibrillation). Two sample t-tests were used to compare the number of errors in total and in each of the 4 areas (ventilation, compressions, medication administration, and defibrillation) between the two groups. Types of errors were described and identified from the video recordings.

3. Performance of Technical Skills (means scores for ventilation, compressions, medication administration, defibrillation, and total scores). Two sample t-tests were used to compare the total mean scores in each of the 4 areas (ventilation, compressions, medication administration, and defibrillation) and total mean scores between the two groups (control and intervention). 4. Team performance (problem solving, situational awareness, leadership, resource utilization, and communication). Two sample t-tests and chi-square analysis were used to compare team performance scores in each of the 5 areas and in total mean scores to identify a difference between the two groups (control and intervention). 


\section{Chapter IV}

\section{Results}

The primary aim of this quasi experimental study was to test a color-coded SMOCK system on time to implement four primary emergency patient care measures, identify any medical errors committed, and evaluate team performance measures during simulated cardiopulmonary resuscitation (CPR) exercises.

The study was designed to address the following research questions: Between treatment and control groups (1) was there a difference in time to implement primary emergency measures for ventilation, compressions, medication administration and defibrillation? (2) Was there a difference in the number of recorded medical errors (i.e. sequence in initiating ventilation and compression; medication administration [giving the wrong medication via the wrong route,]; omission of ventilation, compressions, requested medications or defibrillation; or errors in providing ventilation, compressions, or defibrillation)? (3) Was there a difference in performing the number of required technical skills using mean scores for ventilation, compression, medication administration, and defibrillation) for successful resuscitation outcomes. (4) Was there a difference in team performance measures when using five key components (problem solving, situational awareness, leadership, resource utilization, and communication) of a crisis resource management tool? This chapter presents the characteristics of the sample and the results of the data analysis for each research question.

\section{Sample}

Following IRB approval from FIU and UM-JMH, a total of 260 nurses from a private hospital, a public tertiary hospital, and two nursing schools (training facility at public hospital) were recruited for the study. The nurses were allocated to one of two study arms (control or 
intervention), and each arm consisted of 20 groups of 5-8 individuals. Although, the study was designed to have eight distinctive roles assigned for each group to measure CPR performance, the group size varied between five to eight member teams as they reported for CPR recertification or code training.

Recruitment for the entire study sample was planned to come from a convenience sample of nurses training at UM-JMH Center for Patient Safety. However due to a freeze on hiring new nurses into the medical-surgical internship program from both facilities, nurses were recruited from other training courses including basic life support classes within the hospital, and from two local nursing schools which use the UM-JMH facility for code training. All participants were RNs. The four primary roles (compressions, ventilations, medication administration, and defibrillation) to be evaluated were assigned as planned.

The changes in recruitment affected the group sample size since program facilitators had already selected the number of students (facilitator: student ratio) to participate in the training exercises. To control for this, an equal number of groups with differing sizes was balanced between the control and intervention groups. For example, if the intervention group had three groups of five participants, and the control group also had three groups with five participants. The total number of study participants was the same for both the control and the intervention group (130 nurses in each group).

The mean age for the total sample of 260 nurses enrolled in this study was 40 years $(\mathrm{SD}=11.6)($ Table 1$)$. There was no significant difference $(\mathrm{t}=-1.2, \mathrm{p}=0.24)$ between the mean age of the control group $(\mathrm{M}=39.2, \mathrm{SD}=11.04)$ and the intervention group $(\mathrm{M}=41.0, \mathrm{SD}=12.16)$. The majority of the sample was female (200) with 60 male participants. The control group consisted of 102 females (78\%) and 28 males (22\%) while the intervention group had 98 females (75\%) 
and 32 male (25\%) participants. The groups were not significantly different for gender $\left(\chi^{2}=.35\right.$, $\mathrm{p}>.05)$.

Overall nurses listed their highest level of education as an associate degree (74), bachelor's degree (123), and a master's degree (13) or did not list a degree status (50). In the control group there were 28 nurses (22\%) with an associate degree, 77 nurses (59\%) with a bachelor's degree, five nurses (4\%) with a master's degree, and 20 nurses (15\%) who did not report a degree. Additionally, in the intervention group there were 46 nurses (35\%) with an associate degree, 46 nurses (35\%) with a bachelor's degree, eight nurses (7\%) with a master's degree, and 30 nurses (23\%) who did not list a degree. There was a significant difference $\left(\chi^{2}\right.$ $=14.88, \mathrm{p}=.002)$ in the number of nurses in the control group holding a bachelor's degree compared to those in the intervention group.

Every year a large number of healthcare professionals change careers due to the constant availability of nursing positions in a variety of healthcare settings. Therefore, the study sought to capture this information on the demographic survey, and the results indicated that nurses in the study worked a mean of $9.4(\mathrm{SD}=10.03)$ years in any healthcare position. There was no statistically significance difference $(t=.70, p>.05)$ between the control $(\mathrm{M}=9.8, \mathrm{SD}=9.8)$ and the intervention group $(\mathrm{M}=9.0, \mathrm{SD}=10.24)$ in years worked in a health care position. Participants in this study worked a mean of $7.3(\mathrm{SD}=9.4)$ years in a nursing position. There was no statistically significant difference $(\mathrm{t}=.83, \mathrm{p}>.05)$ between the control $(\mathrm{M}=7.4, \mathrm{SD}=10.31)$ and the intervention $(\mathrm{M}=7.1, \mathrm{SD}=8.5)$ group in years worked in a nursing position.

In this study, 220 (85\%) nurses' last CPR re-certifications were current and completed within the recommended window of two years set by the American Heart Association (AHA). Eighteen nurses (14\%) in the control group and 22 nurses (17\%) in the intervention group listed 
their BLS re-certification date out of the AHA recommended window or did not report their last re-certification status. In 2012, only 40 nurses (15\%) completed their CPR recertification, twelve $(9 \%)$ in the control group and $37(28 \%)$ in the intervention group. Most nurses $(125,48 \%)$ in this study completed their recertification in 2011,79 nurses $(61 \%)$ in the control group and 46 nurses (35\%) in the intervention group. In 2010, only 46 nurses (18\%) completed their CPR recertification as recommended by the AHA, 21 nurses (16\%) in the control group and $25(19 \%)$ in the intervention group. Nurses were asked to list the number of times they had ever participated in a mock code. 155 nurses $(60 \%)$ had participated in at least one mock code while $104(40 \%)$ nurses had never done so. In the control group 83 nurses (64\%) participated in one or more mock codes while 46 (36\%) had never participated in a mock code. In the intervention group 72 nurses $(55 \%)$ had participated in at least one or more mock codes while 58 nurses $(45 \%)$ had never participated in a mock code. There was no significant difference $\left(\chi^{2}=11.95\right.$, p>.05) between the two groups' mock code experience.

In this study, 149 nurses (57\%) reported that they had participated in an actual hospital code event, 74 (57\%) in the control group and 75 (58\%) in the intervention group. There was no significant difference between the two groups' real code experiences.

Seventy two (28\%) nurses reported that they had provided CPR measures to individuals outside of a hospital setting, 33 nurses (25\%) in the control group and 39 nurses $(30 \%)$ in the intervention group; $185(72 \%)$ nurses indicated that they had never done so. There was no significant difference between the two groups on out of hospital experience (table 1). 
Table 1 Sample Characteristics

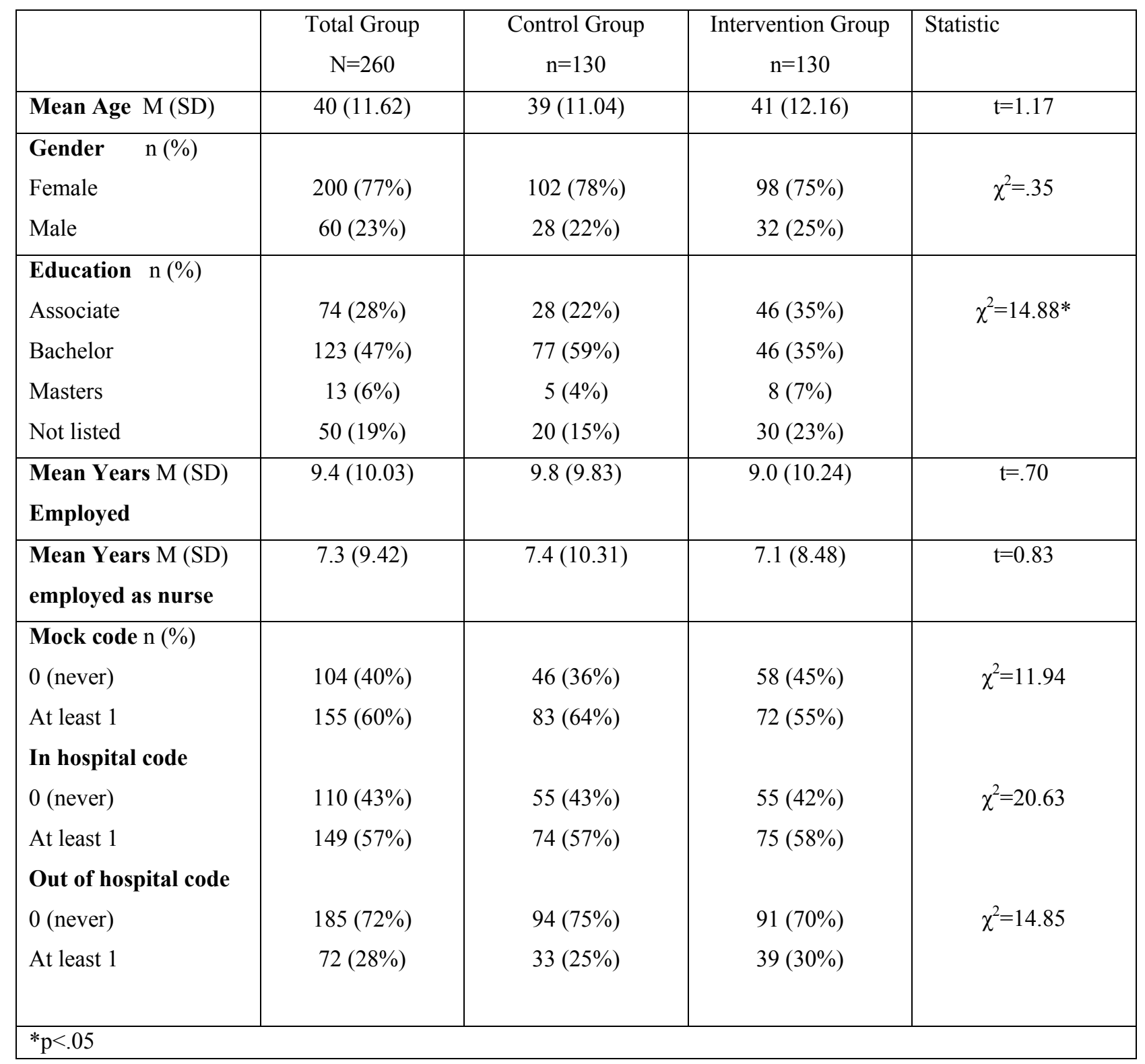

Two hundred seventeen nurses (83\%) indicated that they felt comfortable performing

basic CPR skills while 43 nurses (17\%) reported they did not (Table 2). Three nurses (1\%)

provided no data. In the control group 108 nurses (83\%) indicated comfort performing CPR

while 22 nurses (17\%) were not comfortable and 3 nurses (2\%) did not respond to this question.

Results were similar for the intervention group with 109 nurses (84\%) reporting CPR comfort 
while 21 nurses $(16 \%)$ did not. There was no significant difference $\left(\chi^{2}=3.10, p>.05\right)$ between the two groups in their CPR comfort level.

In examining perceived strengths in CPR skills, 183 (84\%) nurses in the total group felt comfortable performing compressions. There was no significant difference $\left(\chi^{2}=.02, p>.05\right)$ between the control 91 (49\%) and the intervention 92 (51\%) groups. From the total of 122 nurses (47\%) responding to comfort with bag-mask ventilation, 70 nurses (57\%) in the control group felt comfortable with this skill compared to 52 nurses (43\%) in intervention group. The difference was significant $\left(\chi^{2}=5.0, p=0.02\right)$.

Further examination of the strengths of the total group of nurses $138(54 \%)$ responding to medication administration revealed that nurses were not very comfortable giving medication. Only 64 nurses (49\%) in the control group and 74 nurses (57\%) in the intervention group reported that they felt comfortable administering medications during a code. The results were not significant between the two groups $\left(\chi^{2}=1.6, \mathrm{p}>.05\right)$.

A total of 132 nurses (51\%) responded to their comfort level with the use of a defibrillator. Only 62 nurses (48\%) in the control group and 70 nurses (54\%) in the intervention group reported that they felt comfortable using a defibrillator during a code. There were no significant differences $\left(\chi^{2}=1.0, \mathrm{p}>.05\right)$ between the groups.

In examining perceived weaknesses in CPR skills among the total group of nurses, only 36 nurses (14\%) indicated that they did not feel comfortable providing high quality compressions. There was not a significant difference $\left(\chi^{2}=.01, \mathrm{p}>.05\right)$ between the 18 nurses (14\%) from the control and the 18 nurses (14\%) in the intervention group who reported low comfort levels when providing compressions. The results were very similar for ventilations with only 37 nurses (14\%) in the total group reporting that they did not feel comfortable using the 
bag-mask device. These results were not significantly different $\left(\chi^{2}=1.4, p>.05\right)$ with 22 nurses $(17 \%)$ in the control group and 15 nurses $(12 \%)$ in the intervention group who felt uncomfortable. Out of the 126 nurses (48\%) who indicated that medication administration was a weakness, there were 63 nurses (50\%) in each group who did not feel comfortable administering medication during a code event. These group differences were not significant $\left(\chi^{2}=.03, \mathrm{p}>.05\right)$. A large number of nurses $86(34 \%)$ in this sample did not feel comfortable using the defibrillator, 42 nurses (32\%) in the control group and 44 nurses (36\%) in the intervention group. The differences were not significant $\left(\chi^{2}=.16, \mathrm{p}>.05\right)$ (table 2). 
Table 2 Self Reported CPR Skill Strength and Weakness among Participants

\begin{tabular}{|c|c|c|c|c|}
\hline $\begin{array}{l}\text { Skill } \\
\text { Comfort level }\end{array}$ & $\begin{array}{c}\text { Total Group } \\
\mathrm{N}=260\end{array}$ & $\begin{array}{c}\text { Control Group } \\
n=130\end{array}$ & $\begin{array}{l}\text { Intervention Group } \\
n=130\end{array}$ & $\chi^{2}$ Statistic \\
\hline \multicolumn{5}{|l|}{ Adequate overall } \\
\hline Skills $\quad \mathrm{n}(\%)$ & & & & \multirow{3}{*}{3.1} \\
\hline Yes & $217(83 \%)$ & $108(83 \%)$ & $109(84 \%)$ & \\
\hline No & $43(17 \%)$ & $22(17 \%)$ & $21(16 \%)$ & \\
\hline \multicolumn{5}{|c|}{ Strengths } \\
\hline Compressions $\mathrm{n}(\%)$ & & & & \multirow{3}{*}{.02} \\
\hline Yes & $183(84 \%)$ & $91(49 \%)$ & $92(51 \%)$ & \\
\hline No & $75(36 \%)$ & $38(51 \%)$ & $37(49 \%)$ & \\
\hline \multicolumn{5}{|l|}{ Ventilation $\quad \mathrm{n}(\%)$} \\
\hline Yes & $122(47 \%)$ & $70(57 \%)$ & $52(43 \%)$ & \multirow[t]{2}{*}{$5.0^{*}$} \\
\hline No & $136(53 \%)$ & $59(43 \%)$ & $77(57 \%)$ & \\
\hline \multicolumn{5}{|l|}{ Medication $\quad \mathrm{n}(\%)$} \\
\hline Yes & $138(54 \%)$ & $64(49 \%)$ & $74(57 \%)$ & \multirow[t]{2}{*}{1.6} \\
\hline No & $120(46 \%)$ & $65(50 \%)$ & $55(43 \%)$ & \\
\hline \multicolumn{5}{|l|}{ Defibrillation $\mathrm{n}(\%)$} \\
\hline Yes & $132(51 \%)$ & $62(48 \%)$ & $70(54 \%)$ & \multirow[t]{2}{*}{1.0} \\
\hline No & $126(48 \%)$ & $67(52 \%)$ & $59(45 \%)$ & \\
\hline \multicolumn{5}{|c|}{ Weaknesses } \\
\hline Compressions $\mathrm{n}(\%)$ & & & & \multirow{3}{*}{.01} \\
\hline Yes & $36(14 \%)$ & $18(14 \%)$ & $18(14 \%)$ & \\
\hline No & $221(85 \%)$ & $112(86 \%)$ & $109(84 \%)$ & \\
\hline \multicolumn{5}{|l|}{$\begin{array}{ll}\text { Ventilation } & \mathrm{n}(\%)\end{array}$} \\
\hline Yes & $37(14 \%)$ & $22(17 \%)$ & $15(12 \%)$ & \multirow[t]{2}{*}{1.4} \\
\hline No & $220(85 \%)$ & $108(83 \%)$ & $112(86 \%)$ & \\
\hline \multicolumn{5}{|l|}{ Medication $\quad \mathrm{n}(\%)$} \\
\hline Yes & $126(48 \%)$ & $63(49 \%)$ & $63(49 \%)$ & \multirow[t]{2}{*}{.03} \\
\hline No & $131(52 \%)$ & $67(51 \%)$ & $64(51 \%)$ & \\
\hline \multicolumn{5}{|l|}{ Defibrillation $\mathrm{n}(\%)$} \\
\hline Yes & $86(34 \%)$ & $42(32 \%)$ & $44(36 \%)$ & \multirow[t]{2}{*}{.16} \\
\hline No & $171(66 \%)$ & $88(68 \%)$ & $83(64 \%)$ & \\
\hline
\end{tabular}


Research question number one Were there differences in time to implement primary emergency measures for ventilation, compressions, medication administration, and defibrillation between the control and intervention (smock) groups?

The time to implement primary CPR performance measures was captured from video recordings and scored using the most current AHA BLS/ACLS performance criteria and outcome measure for cardiac arrest response. The start time to begin each CPR performance measure began when the patient lost consciousness and ended five minutes after the patient became asystolic regardless of the length of the resulting scenario. The actual time in seconds was compared in the control and intervention groups for each primary task (ventilation, compression, medication administration, and defibrillation). The time in seconds for each task was summed for each group and the mean of the total summed scores were compared.

The mean time for the total sample to start compressions once the patient loss his level of consciousness was $63.40(\mathrm{SD}=54.00)$ seconds. The control group mean time of 86.90 ( $\mathrm{SD}=61.16)$ seconds took twice as long to get stated compared to the intervention group men time $39.90(\mathrm{SD}=32.89)$ seconds. There was a statistically significant difference between these two groups ( $\mathrm{t}=3.0, \mathrm{p}=0.005$ ). Additionally, the mean time nurses overall took to start bag mask ventilation was about $77.55(\mathrm{SD}=62.79)$ seconds. Again, the control group mean time of 103.65 (SD 69.12) seconds took almost twice as long to give the first breath when compared to the intervention group $51.45(\mathrm{SD}=43.38)$ seconds. These results were significantly different when using the independent $\mathrm{t}$-test $(\mathrm{t}=2.86, \mathrm{p}=0.004)$.

The mean time to administer the first dose of medication was $44.25(\mathrm{SD}=78.51)$ seconds for the total sample. The mean time for the control group was $80.19(\mathrm{SD}=17.93)$ seconds compared to the intervention group $79.57(\mathrm{SD}=17.80)$. There were no significant differences 
between the control and intervention groups for time to administer medication $(\mathrm{t}=-.03, \mathrm{p}>.05)$.

Results indicated that in the total sample, nurses' mean time to operate the defibrillator (AED) was $129.87(\mathrm{SD}=71.89)$ seconds. Mean time for the control group was $151.85(\mathrm{SD}=72.36)$ seconds, slightly longer than the intervention group mean time of $107.90(\mathrm{SD}=66.00)$. This was significantly different between groups $(\mathrm{t}=2.00, \mathrm{p}=0.05)$ (table 3$)$.

Table 3 Time in seconds to implement Primary Emergency Measures

\begin{tabular}{|l|c|c|c|c|}
\hline $\begin{array}{l}\text { Mean Time in } \\
\text { Seconds }\end{array}$ & $\begin{array}{c}\text { Total Group } \\
\mathrm{N}=40\end{array}$ & $\begin{array}{c}\text { Control Group } \\
\mathrm{n}=20\end{array}$ & $\begin{array}{c}\text { Intervention Group } \\
\mathrm{n}=20\end{array}$ & t value \\
\hline $\begin{array}{l}\text { Compressions } \\
\text { M (SD) }\end{array}$ & $63.40(54.00)$ & $86.90(61.16)$ & $39.90(32.89)$ & $3.02 *$ \\
\hline $\begin{array}{l}\text { Ventilation } \\
\text { M (SD) }\end{array}$ & $77.55(62.79)$ & $103.65(69.12)$ & $51.45(43.38)$ & $2.87 *$ \\
\hline $\begin{array}{l}\text { Medication } \\
\text { M (SD) }\end{array}$ & $44.25(78.51)$ & $80.19(17.93)$ & $79.57(17.80)$ & -.032 \\
\hline $\begin{array}{l}\text { Defibrillation } \\
\text { M (SD) }\end{array}$ & $129.87(71.89)$ & $151.85(72.36)$ & $107.90(66.00)$ & $2.00 *$ \\
\hline$* \mathrm{p}<.05$ & & & \\
\hline
\end{tabular}

Research question number two Was there a difference in the number of recorded medical errors

(i.e. sequence in initiating ventilation and compression; medication administration [giving the wrong medication via the wrong route]; omission of ventilation, compressions, requested medications or defibrillation; or errors in providing ventilation, compressions, or defibrillation) between the two groups?

The total number of medical errors was captured by counting the number/frequency of incorrect tasks in CPR technical skills performance by each group. These medical errors were: any improper use of the bag mask ventilation device (i.e. wrong size mask, unable to obtain a seal, and no chest rise with each ventilation breath); giving medications not according to CPR recommendations (i.e. giving the wrong medication via the wrong route, omission of a dose, or any medication requested but not given); and not applying compressions at a depth of 2 inches or 
$5 \mathrm{~cm}$ or at a rate of at least 100/minute (incorrect hand placement) that is not consistent with the 2010 AHA guidelines. Medical errors in use of the defibrillator during CPR training included incorrect placement of pads along with setting an incorrect amount of voltage, or unable to set the machine to apply cardio version and/or defibrillation when using the life pack-20 device.

The total number of medical errors associated with each task was captured from the technical skill score sheet. A score of two indicated that there were no errors made for a specific task. A score of one or zero indicated that an error was made because the task was incompletely performed or omitted. Results revealed that errors occurred in all of the four primary CPR measures for both the control and intervention groups. There were a total of 22 group (55\%) errors in providing compressions once the patient loss consciousness (LOC). The control group of nurses had more errors $16(80 \%)$ providing compressions when compared to the intervention group of $6(30 \%)$ errors. There was a significant difference $\left(\chi^{2}=10.10, p=0.005\right)$ between the number of errors related to compressions for the control group compared to the intervention group.

Results were very similar for the number of errors made by the total group $22(55 \%)$ of nurses performing proper bag mask ventilation. The total group scores were different for the control $17(85 \%)$ and intervention group $5(25 \%)$. There was a significant difference $\left(\chi^{2}=3.1\right.$, $\mathrm{p}=0.001$ ) between the number of errors related to proper bag mask ventilation for the control and the intervention group.

Out of the 40 groups, 34 groups (85\%) made errors while administering medication. The number of errors between the control $15(75 \%)$ and intervention $19(95 \%)$ groups were not significantly different $\left(\chi^{2}=14.54, p>.05\right)$. A similar trend was seen with the improper use of the defibrillator among the total group of nurses $15(38 \%)$ errors. The number of errors in the 
control $10(50 \%)$ and intervention $5(25 \%)$ groups while using the defibrillator indicated there was no significant difference $\left(\chi^{2}=2.7, p>.05\right)$ in errors between groups.

The mean summed scores of all errors combining the four categories (compressions, ventilation, medication administration, and defibrillation) indicated a total group mean error score of $6.58(\mathrm{SD}=1.97)$. This was slightly lower than the control group mean error score of 7.55 $(\mathrm{SD}=1.54)$, and slightly higher than the intervention group mean error score $5.60(\mathrm{SD}=1.90)$.

Overall there was a significant difference $(\mathrm{t}=-2.6, \mathrm{p}=0.01)$ between the summed total mean errors scores for the control and intervention group. These results suggest that the use of assigned roles in the intervention group helped reduced the number of errors when compared to the control group or standard of care (Table 4).

Table 4 Number of Errors for CPR Measures

\begin{tabular}{|c|c|c|c|c|}
\hline $\begin{array}{l}\text { Group } \\
\text { Skill Error }\end{array}$ & $\begin{array}{l}\text { Total Group } \\
\qquad \mathrm{N}=40\end{array}$ & $\begin{array}{c}\text { Control Group } \\
\qquad \mathrm{n}=20\end{array}$ & $\begin{array}{l}\text { Intervention Group } \\
\qquad \mathrm{n}=20\end{array}$ & Statistic \\
\hline $\begin{array}{l}\text { Compressions n }(\% \\
\text { No } \\
\text { Yes }\end{array}$ & $\begin{array}{l}18(45 \%) \\
22(55 \%)\end{array}$ & $\begin{array}{c}4(2 \%) \\
16(80 \%)\end{array}$ & $\begin{array}{c}14(70 \%) \\
6(30 \%)\end{array}$ & $\chi^{2}=10.10^{*}$ \\
\hline $\begin{array}{l}\text { Ventilation } \\
\text { No } \\
\text { Yes }\end{array}$ & $\begin{array}{l}18(45 \%) \\
22(55 \%)\end{array}$ & $\begin{array}{l}3(15 \%) \\
17(85 \%)\end{array}$ & $\begin{array}{c}15(75 \%) \\
5(25 \%)\end{array}$ & $\chi^{2}=3.1$ \\
\hline $\begin{array}{l}\text { Medication } \\
\text { No } \\
\text { Yes }\end{array}$ & $\begin{array}{c}6(15 \%) \\
34(85 \%)\end{array}$ & $\begin{array}{c}5(25 \%) \\
15(75 \%)\end{array}$ & $\begin{array}{l}1(.05 \%) \\
19(95 \%)\end{array}$ & $\chi^{2}=14.54^{*}$ \\
\hline $\begin{array}{l}\text { Defibrillation } \\
\text { No } \\
\text { Yes }\end{array}$ & $\begin{array}{l}25(63 \%) \\
15(38 \%)\end{array}$ & $\begin{array}{l}10(50 \%) \\
10(50 \%)\end{array}$ & $\begin{array}{l}15(75 \%) \\
5(25 \%)\end{array}$ & $\chi^{2}=2.7$ \\
\hline $\begin{array}{l}\text { Total mean errors } \\
\mathrm{M}(\mathrm{SD})\end{array}$ & $6.58(1.97)$ & $7.55(1.54)$ & $5.60(1.90)$ & $\mathrm{t}=-2.6^{*}$ \\
\hline
\end{tabular}


Research question number three Was there a difference in the number of groups performing the required technical skills using mean scores for ventilation, compression, medication administration, and defibrillation for successful resuscitation outcomes?

The total technical skills score associated with each task for CPR performance was captured from the technical skill score sheet which used the following scoring system. For all tasks, a score of two indicated that the task was performed completely and a score of one represented an incomplete performance of a necessary task. A zero score indicated that there was no attempt to perform all the steps necessary for the required task.

The scores for the total sample for performing compressions correctly on the patient were seen in 18 groups. In the control arm only 4 groups (20\%) performed the task correctly and 14 groups $(70 \%)$ in the intervention arm performed the task correctly. There was a significant difference $\left(\chi^{2}=10.4, p<.005\right)$ in number of groups performing compressions according to the AHA standards between the control and intervention group with the intervention group performing better.

The technical skills score for using a bag mask ventilation device correctly to provide the first breath with good chest rise was assigned a score of two. A total of 18 groups (45\%) of nurses used the bag mask device correctly when providing adequate ventilation to the patient. The control arm had only three groups (15\%) that used the bag mask device correctly when compared to 15 groups $(75 \%)$ in the intervention arm. There was a significant difference $\left(\chi^{2}=14.8, p<0.001\right)$ in group performance between the intervention and control group. Nurses in the intervention group performed better technical skills when using the bag mask device.

Most groups (85\%) did not provide or suggest the use of medications for the patient even though they were aware of the AHA medication algorithm. Medication administration in the total 
sample was lower than the expected and nurses from 15 (75\%) of the control groups and 19 $(95 \%)$ of the intervention groups did not demonstrate proficiency of skills. Additionally, only five groups $(25 \%)$ in the control arm and one group (5\%) in the intervention arm correctly administered medication to the patient. Although most groups did not administer medication, there was a significant difference $\left(\chi^{2}=6.6, p<.05\right)$ between the performance of medication administration between the control and intervention groups.

Results regarding the use of the defibrillator were better than those seen with medication administration. More than half (63\%) of the groups were able to turn on the defibrillator, apply the pads, and deliver the first shock as recommended by the AHA. Ten groups $(50 \%)$ in the control arm and 15 groups (75\%) in the intervention arm demonstrated proficient skills with the defibrillator. There was no statistically significant $\left(\chi^{2}=3.2, p>.05\right)$ difference between the control and the intervention group when demonstrating the proper use of the defibrillator during a code event.

The mean summative scores of all technical skills for each of the four categories (compressions, ventilation, medication administration, and defibrillation) for the total sample was 17.05 ( $\mathrm{SD}=2.71)$; for the control group $16.00(\mathrm{SD}=2.58)$, and for the intervention group $18.10(\mathrm{SD}=2.51)$. These means were statistically significant $(\mathrm{t}=-2.61, \mathrm{p}=0.01)$ between the control and intervention group suggesting that assigned roles may help nurses focus on the technical skills needed to correctly complete an important task during resuscitation (table 5). 
Table 5 Technical Skills CPR Performance Scores

\begin{tabular}{|c|c|c|c|c|}
\hline $\begin{array}{l}\text { Numeric score } \\
0,1,2\end{array}$ & $\begin{array}{l}\text { Total Group } \\
\mathrm{N}=40\end{array}$ & $\begin{array}{l}\text { Control Group } \\
\mathrm{N}=20\end{array}$ & $\begin{array}{l}\text { Intervention Group } \\
\mathrm{N}=20\end{array}$ & Statistic \\
\hline \multicolumn{5}{|l|}{ Compressions n (\%) } \\
\hline No attempt & $1(2 \%)$ & $1(5 \%)$ & 0 & $\chi^{2}=10.4^{*}$ \\
\hline Partial & $21(53 \%)$ & $15(75 \%)$ & $6(30 \%)$ & \\
\hline Completed & $18(45 \%)$ & $4(20 \%)$ & $14(70 \%)$ & \\
\hline \multicolumn{5}{|l|}{ Ventilation $\quad \mathrm{n}(\%)$} \\
\hline No attempt & $1(3 \%)$ & $1(5 \%)$ & 0 & $\chi^{2}=14.8^{*}$ \\
\hline Partial & $21(52 \%)$ & $16(80 \%)$ & $5(25 \%)$ & \\
\hline Completed & $18(45 \%)$ & $3(15 \%)$ & $15(75 \%)$ & \\
\hline \multicolumn{5}{|l|}{$\begin{array}{ll}\text { Medication } & \mathrm{n}(\%)\end{array}$} \\
\hline No attempt & $31(78 \%)$ & $15(75 \%)$ & $16(80 \%)$ & $\chi^{2}=6.6$ \\
\hline Partial & $3(7 \%)$ & 0 & $3(15 \%)$ & \\
\hline Completed & $6(15 \%)$ & $5(25 \%)$ & $1(5 \%)$ & \\
\hline \multicolumn{5}{|l|}{ Defibrillation n (\%) } \\
\hline No attempt & $5(13 \%)$ & $4(20 \%)$ & $1(5 \%)$ & $\chi^{2}=3.2$ \\
\hline Partial & $10(25 \%)$ & $6(30 \%)$ & $4(20 \%)$ & \\
\hline Completed & $25(62 \%)$ & $10(50 \%)$ & $15(75 \%)$ & \\
\hline $\begin{array}{l}\text { Total mean scores } \\
\mathrm{M}(\mathrm{SD})\end{array}$ & $17.05(2.71)$ & $16.00(2.58)$ & $18.10(2.51)$ & $\mathrm{t}=-2.6^{*}$ \\
\hline
\end{tabular}

Research question number four Was there a difference in team performance measures when

using five key components (problem solving, situational awareness, leadership, resource utilization, and communication) of a crises resource management tool?

Team performance was captured with five items (problem solving, situational awareness, leadership, resource utilization, and communication) on the Ottawa Abbreviated Global Rating Scale (AGRS) checklist which has been used to assess five categories of crises resource management and team performance. The checklist used a six point scoring system to indicate whether various team performance components were demonstrated during the scenario. Each task was given a numeric score from zero to two based upon performance of each task. A score 
of two was given when the team fully demonstrated all the components of a particular team performance measure from the AGR checklist. A one was assigned when the task was partially completed. A score of zero was assigned if no items on the checklist were performed. Scores for each task were summed and then means for each task were calculated. The mean summative score was $16.13(\mathrm{SD}=2.32)$ for the total sample. Mean summative score for the control 15.25 $(\mathrm{SD}=2.09)$ and intervention $17.00(\mathrm{SD}=2.25)$ groups were significantly different $(\mathrm{t}=-2.54, \mathrm{p}=.02)$ with the intervention performing better.

The total of 20 groups performance score was adequate for problem-solving (i.e., patient assessment and provided concurrent management) related to CPR performance measures was slightly higher than both the control (9) groups and the intervention (10) groups. These results did not show any significant difference in problem-solving techniques between the control and the intervention groups $\left(\chi^{2}=3.7, p>.05\right)$. A total of 38 groups represented that nurses were able to do some aspects of situational awareness (i.e., fixation errors and patient condition). Eighteen groups in the control arm and 20 groups in the intervention arm which not significantly different $\left(\chi^{2}=5.0, p>.05\right)$ from each other. Results imply that both groups were able to avoid fixation errors and reassess the patient's condition in a similar fashion. The 13 groups from the total sample represented the successful use of the resource performance measure (i.e., call for help and delegate tasks). The results were differences between the control group with 2 groups and the intervention group had 11 groups use resources appropriately. These results showed a significant difference $\left(\chi^{2}=11.2, p=0.03\right)$ between the groups. This suggested that nurses in the intervention group were more likely to call for help and delegate tasks effectively.

Thirty six of the 40 groups (90\%) showed a varying degree of leadership skills. There were 20 groups in the control arm and 16 groups in the intervention arm that demonstrated some 
form of leadership skills. There was no significant difference $\left(\chi^{2}=10.6, p>.05\right)$ between groups regarding leadership skills. In both groups, as per the scoring tool, the team leader did not maintain the global perspective, act decisively to control the crisis, or maintain a calm demeanor throughout each scenario. The scores for communication were similar to the leadership scores with 38 out of 40 (95\%) groups using some form of communication skills. The control arm had 20 groups and the intervention arm had 16 groups that did not communicate very well which were not statistically different $\left(\chi^{2}=5.8, p>.05\right)$. In both groups, as per the score sheets, team leadership skills were mediocre because the groups did not communicate clearly to team members, use close loop communication techniques, and did not offer or listen to team input. 
Table 6 Mean Scores for Team Performance

\begin{tabular}{|c|c|c|c|c|}
\hline Numeric scores & $\begin{array}{l}\text { Total Group } \\
\quad \mathrm{N}=40\end{array}$ & $\begin{array}{c}\text { Control Group } \\
n=20\end{array}$ & $\begin{array}{l}\text { Intervention } \\
\text { Group } n=20\end{array}$ & Statistic \\
\hline $\begin{array}{l}\text { Problem Solving } \quad \mathrm{n}(\%) \\
\text { No attempt } \\
\text { Partial } \\
\text { Completed }\end{array}$ & $\begin{array}{c}0 \\
20(50 \%) \\
20(50 \%)\end{array}$ & $\begin{array}{c}0 \\
9(45 \%) \\
11(55 \%)\end{array}$ & $\begin{array}{c}0 \\
11(55 \%) \\
9(45 \%)\end{array}$ & $\chi^{2}=3.7$ \\
\hline $\begin{array}{l}\text { Situational Awareness } \mathrm{n}(\%) \\
\text { No attempt } \\
\text { Partial } \\
\text { Completed }\end{array}$ & $\begin{array}{c}2(5 \%) \\
28(70 \%) \\
10(25 \%)\end{array}$ & $\begin{array}{l}2(10 \%) \\
12(60 \%) \\
6(30 \%)\end{array}$ & $\begin{array}{c}0 \\
16(80 \%) \\
4(20 \%)\end{array}$ & $\chi^{2}=5.0$ \\
\hline $\begin{array}{l}\text { Resource utilization } \\
\text { No attempt } \\
\text { Partial } \\
\text { Completed }\end{array}$ & $\begin{array}{c}0 \\
27(68 \%) \\
13(33 \%)\end{array}$ & $\begin{array}{c}0 \\
18(90 \%) \\
2(10 \%)\end{array}$ & $\begin{array}{c}0 \\
9(45 \%) \\
11(55 \%)\end{array}$ & $\chi^{2}=11.2 *$ \\
\hline $\begin{array}{l}\text { Leadership } \\
\text { No attempt } \\
\text { Partial } \\
\text { Completed }\end{array}$ & $\begin{array}{c}0 \\
36(90 \%) \\
4(10 \%)\end{array}$ & $\begin{array}{c}0 \\
20(100 \%) \\
0\end{array}$ & $\begin{array}{c}0 \\
16(80 \%) \\
4(20 \%)\end{array}$ & $\chi^{2}=10.6$ \\
\hline $\begin{array}{l}\text { Communication } \\
\text { No attempt } \\
\text { Partial } \\
\text { Completed }\end{array}$ & $\begin{array}{c}0 \\
38(95 \%) \\
2(5 \%)\end{array}$ & $\begin{array}{c}0 \\
20(100 \%) \\
0\end{array}$ & $\begin{array}{c}0 \\
18(90 \%) \\
2(10 \%)\end{array}$ & $\chi^{2}=5.8$ \\
\hline Total mean scores $\mathrm{M}(\mathrm{SD})$ & $16.13(2.32)$ & $15.25(2.09)$ & $17.00(2.25)$ & $\mathrm{t}=-2.54^{*}$ \\
\hline
\end{tabular}

Summary

The nurses in the sample were mainly female $(77 \%)$ and had a mean age of $40(\mathrm{SD}=11.6)$ years. Most nurses (84\%) in the total sample reported that their CPR certifications were valid and that they also participated in mock codes routinely. There were no differences in the groups' number of years work experience in any healthcare or nursing position. In this study more nurses in the control group had a bachelor's degree when compared to nurses in the intervention arm. Additionally, $212(82 \%)$ nurses stated that they felt comfortable performing the technical skills 
for CPR as recommended by the AHA 2010 guidelines. Approximately 183 (70\%) nurses reported either compressions or ventilations were their greatest strength in CPR technical skills. Nurses also reported their two weakest technical skills as medication administration $\mathrm{n}=126$ $(59 \%)$ and defibrillation $\mathrm{n}=86(41 \%)$.

The study results also showed that nurses in the intervention group were able to start compressions (mean time in 39.9 seconds) in half the time when compared to nurses in the control group (mean time in 86.9 seconds). There were statistically significant differences in time to provide ventilations between the control group (mean time in 103.65 seconds) and the intervention group (mean time in 51.45 seconds). Both groups showed similar performances for time to administer medications. Speed of use of the defibrillator was statistically different between the groups. It took a mean time of 151.85 seconds in the control group and a mean time of 107.90 seconds in the intervention group for nurses to turn on the defibrillator, place pads, and deliver the first shock.

The results of this study also revealed that nurses made a mean of 6.6 errors in performing the necessary technical skills for CPR. The control group made significantly more total errors than the intervention group. Nurses in the intervention group made fewer errors when performing compressions, providing ventilations, and using the defibrillator than the control group. However, nurses in the control and intervention group made about the same number of errors administering medication. Additionally, nurses in the intervention group were similar to nurses in the control group for completing technical skills (18 vs. 16). Nurses in the intervention group were faster and completed more tasks for compressions and ventilations when compared to the control group. However, nurses in the control group were similar with medication administration and defibrillator use when compared to nurses in the intervention group. 
The results of this study did not show many differences in the five categories (problem solving, situational awareness, resource utilization, leadership, and communication) used to assess team performance between the two groups. However, the intervention groups used fewer resources than the control groups. This may be related to individuals in the smock intervention group being more visible and the fact that they had pre-assigned roles. 


\section{Chapter V}

Although, the 2010 American Heart Association (AHA) basic life support recommended guidelines were improved over previous versions, the rates of survival of victims of sudden cardiac arrest (SCA) using CPR have shown little improvement. The 2007 CPR registry report indicated an $18 \%$ survival rate among adults and $27 \%$ survival among pediatric hospitalized patients (CPR registry, 2008). The 2011 Centers for Disease Control and Prevention (CDC) morbidity and mortality report on CPR out of hospital through hospital discharge revealed that rates of survival remain low. Among adults, the survival rate to hospital admission was $26.3 \%$, and the overall survival rate to hospital discharge was 9.6\% (CDC, 2011). Despite decades of research, median reported rates of survival to hospital discharge from SCA using CPR are poor (7.9\%) and have remained virtually unchanged for three decades (CDC, 2011). Since registered nurses (RNs) comprise the largest group of healthcare providers in U.S. hospitals, (U.S. Labor Bureau, 2010) it is imperative that they are competent in performing the four primary measures (compression, ventilation, medication administration, and defibrillation) of CPR to improve survival rates of sudden cardiac arrest (SCA) patients.

\section{Characteristics of Nurses in the Sample}

Currently, there are 2,909,357 registered nurses (RNs) in the U.S. and only five percent of this population is male (Minority nurse, 2012). Additionally, the average age of RNs nationwide is 46.8 years and only eight percent are under 30 years of age (Minority nurse, 2012). The characteristics of this study sample were similar to national averages with a mean age of 40 (SD11.6) years. Twenty percent of the sample was male indicating a greater percent of males compared to the national average of $5 \%$. 


\section{Employment and education}

The educational level for RNs consists of one of three paths: a bachelor's of science in nursing $(\mathrm{BSN})$, an associate degree in nursing ( $\mathrm{ADN}$ ) or a diploma from an approved nursing program (U.S. Bureau of Labor, 2010). These programs range from two to five years for successful completion. However, licensed graduates from any of the three types of programs may qualify for an entry level position as a nurse. Most entry level position nurses are from ADN programs and typically work in a hospital setting (U.S. Bureau of Labor, 2010). Nurses graduating with a BSN or higher degree often take positions in administration, research, consulting, or teaching (U.S. Bureau of Labor, 2010).

According to the 2010 U.S. Department of Health and Human Services Health Resources and Services Administration report, $45.4 \%$ of all RNs initial education was an ADN, 33.7\% was a bachelors' degree and $20.4 \%$ graduated with a diploma (USDHHS-HRSA: The registered nurse population 2010). Only four percent of RN's graduated with a masters' degree. In this study $47 \%$ of the RNs had a bachelor's degree versus $33.7 \%$ nationally, $28 \%$ had an associate degree versus $45.4 \%$ nationally, $5 \%$ a master's degree versus $4 \%$ nationally, and $19 \%$ did not report their degree status. However, most nurses in this sample were not at the entry level position or they may have worked in another healthcare profession prior to becoming a nurse. In this sample, the mean number of years registered nurses worked was $7.3(\mathrm{SD}=9.4)$ with a mean of $9.4(\mathrm{SD}=10)$ years in any healthcare position not necessarily as a nurse.

\section{Certification}

Data on nurses' certification, recertification, and actual CPR experience and comfort level in performing CPR is limited. In a study by Verplancke et al., (2008) using a skill reporter Resusci-Anne mannequin during a two minute BLS performance skills check, $82 \%$ of the nurses 
were female with a mean age of 37 years and a mean of 14 years of work experience. Most nurses' last recertification course occurred more than 16 months prior to the study and 59 months prior to their last CPR experience. Fifty percent of the nurses in Verplancke et al., (2008) study did not have any real life CPR experience. Forty nine percent of the study sample (296) scored their confidence level for performing CPR as one (very weak) while $48 \%$ scored their confidence at level two or three. Only two nurses $(0.006 \%)$ were very confident in their CPR skills and scored their confidence level at four (very good) (Verplancke et al., 2008).

Male nurses' compression depths were deeper (correct depth) than female nurses, and they performed overall compressions significantly better. A shorter time since last BLS training was associated with a greater number of correct ventilations/compression ratios (Verplancke et al., 2008). This study indicated that male gender, self-confidence, and recent BLS training were associated with good quality BLS.

U.S. healthcare professionals including doctors, nurses, and paramedics reported a mean of 16.3 years with a valid certification to perform CPR based on a multi-national attitude survey (IPOS Health, 2009). These healthcare providers reported that they worked a mean of 7.3 years in their current organization, and performed CPR a mean of 19 times within the last 12 months. Seventy five percent perceived that their CPR skill level was high and less than half reported that their organization provided training (mock-codes) beyond the AHA requirements (IPOS Health, 2009).

The results of the present study were similar to both of the above studies with $85 \%$ of the RNs certified to perform CPR with a valid BLS card. However, nearly half (48\%) of the RNs recertification was completed more than one year or beyond the two year AHA recommendations. Only $48 \%$ of the RNs participated in a mock code provided by the hospital 
and $40 \%$ had never participated in a mock code. Fifty eight percent of the RNs participated in a real resuscitation event within the hospital, and $42 \%$ had never participated in a code event either in or outside of the hospital.

Registered nurses in the present study also reported that they rarely perform CPR in an out of hospital setting. Only $29 \%$ had at least one encounter and $71 \%$ never engaged in CPR outside of a hospital. Lipshutz (2008) reported that although, CPR is a simple technique many nurses do not feel comfortable with some of the basic resuscitation procedures. In the present study $83 \%$ of the RNs stated they were comfortable providing CPR. However, $48 \%$ did not feel comfortable administering medication and 33\% did not feel comfortable using the defibrillator. According to the multi-national attitudes survey on CPR, only $75 \%$ of the respondents perceived their level of skill at performing CPR to be good (IPOS Health, 2009). Nearly, all of the respondents reported that high quality CPR is very important to improve patient outcomes from hospital admission through discharge. Continued efforts and frequent training courses are needed to help nurses and other healthcare providers become and remain proficient in CPR performance skills.

\section{CPR Performance Measures}

\section{Compressions}

The 2003 study by Weisfeldt and Becker demonstrated that brief pauses of chest compressions in animals negatively affected hemodynamics during CPR. The effectiveness of chest compressions is dependent upon the rate, depth, pressure, and the technique to generate sufficient flow that improves perfusion (Weisfeldt and Becker, 2003). Hence AHA (2010) guidelines suggest that providing aggressive compressions early is the most important component of CPR to get the heart restarted. 
Abella et al, (2004) used a personal digital assistant (PDA) to record start and end times of each resuscitation event in real-time. Their results showed that the total number of chest compression was too low, chest compression depths were too shallow, and ventilation rates were high (performed at a rate of more than 20/min) (Abella et al., 2004). These ventilation rates were well above the 2005 AHA recommended rate 6-8 breaths/min and chest compression depths of at least 2 inches or $5 \mathrm{~cm}$.

In the study by Valenzuela et al., (2005) chest compressions were only performed $43 \%$ of the time, because EMS providers were frequently interrupted with other resuscitation tasks (setting up the defibrillator or preparing to intubate). The time interval from arrival to first chest compressions was 78 seconds, the longest continuous period of chest compressions was 122 seconds ( 2 minutes), and the shortest period was 11 seconds. These study results indicate that CPR measures from EMS services personnel were initiated in a timely fashion when arrive, however, arrival time was after 6 minutes (heart stops fibrillating), so cardiac compressions should have been started or resumed immediately upon arrival.

The results of the present study were similar to those of Valenzuela et al., (2005) and Abella et al., (2004). In the total sample the mean time for RNs to start compressions was 63 seconds. However, RNs in the intervention group began compressions significantly faster (40 seconds) than the RNs in the control group (87 seconds). These results suggest that pre-assigned roles using visible signage (color-labeled smocks) may be beneficial for code performance and potentially improve SCA patient outcomes.

\section{Ventilations}

In the study by Hunt et al., (2007) ward nurses were involved in 100\% of the mock codes as first responders. The time it took the first responder to enter the room was within 15 seconds, 
and the median time of arrival for any physician was 3 minutes and 6 minutes for the first member of the code team. Further analysis of their results showed that first responders took greater than 1.5 minutes ( 90 seconds) to initiate assessment of airway and breathing, greater than 5 minutes (300 seconds) to initiate bag mask ventilation (BMV), and greater than 7 minutes (420 seconds) to initiate assessment of circulation by checking the pulse.

Results of the present study showed that nurses were faster giving the first breath than the nurses in the Hunt et al., (2007) study. In the present study the mean time for the total group of RNs to begin ventilation using a bag mask device was 78 seconds compared to 300 seconds for the Hunt et al., (2007) study. Additionally, there was a significant difference in the mean time to deliver the first breath between the intervention (51 seconds) and control group (104 seconds). These results also suggest that the use of pre-assigned color-labeled smocks during an emergency may improve time to initiate ventilation because the $\mathrm{RN}$ only had to focus on one task.

\section{Medication Administration}

According to the U.S. pharmacopoeia, nurses commit over $55 \%$ of all medication errors in a hospital setting. Nurses report that these errors are related to numerous distractions and interruptions at the point of care. Pape et al., (2005) study using a standard protocol and visible signage during routine care improved medication delivery by $70 \%$ when a red vest was worn by the nurse preparing and administering medication along with other visible signage such as do not disturb signs. These interventions were very effective in eliminating errors in a hospital setting (Pape et al., 2005).

During medical emergencies, every second counts as patient harm increases and survival decreases. Medications administered during cardiopulmonary arrests (codes) are considered high 
risk drugs which can prove fatal when given inappropriately or by mistake (Kozer et al., 2004). The study by Kozer et al., (2004) showed that physicians initiated 125 orders for drugs. In 24 of the cases the orders were repeated more than once and in 12 cases the ordered drug was not administered. Also, in 21 cases the dose was not specified in the order and in 52 orders the route for administration was not specified (Kozer et al., 2004). This study identified frequent and potentially serious medication errors which occurred at all stages of resuscitation (Kozer et al., 2004).

In the present study nurses did not routinely give medications. Overall, $48 \%$ of the RN sample indicated they did not feel comfortable giving medications. Mean time until the first dose of epinephrine was given was 44 seconds. However, most RN groups (78\%) did not make any attempt to give medication during the mock code training exercises. These results could be due to RNs not routinely giving medications without a physician or advance practice nurse (APN) order. They may not feel comfortable giving medications during a code without the presence of a physician or APN. There were no differences in the performance of medication administration between the RN's in the intervention and control groups.

\section{Defibrillation}

In the study by Valenzuela et al., (2005) results showed that when a code was called, the median time to arrive, diagnose a cardiac rhythm and to treat the rhythm with compressions or defibrillation was 20 seconds. Also, time from arrival to a patient in ventricular fibrillation to delivering the first shock was 54 seconds with an additional 30 seconds between the second and third shocks.

In the Hunt et al. (2009) study nearly $75 \%$ of the medical residents noticed a change in the patient's condition from a normal sinus rhythm to premature ventricular tachycardia (PVT) 
within 9 seconds. The median time before residents asked for the defibrillator was 33 seconds, and only $54 \%$ of residents defibrillated the mannequin in less than 3 minutes of onset of PVT. These residents took a median time of 84 seconds to successfully deliver a shock (Hunt et al., 2009).

In the present study results were different with the total sample of RNs taking a mean time of 129 seconds to turn on the defibrillator, place pads, and deliver the first shock. The time to successfully use the defibrillator was significantly different between the intervention (108 seconds) and the control (152 seconds) group. Although these times were longer than those documented in previous studies, some RNs (33\%) in this study reported that they did not feel comfortable using the defibrillator. Despite these perceptions RNs performed better when allowed to use pre-assign roles. Role assignment using the smocks for CPR technical skills performance may also eliminate medical errors by allowing nurses to select the role in which they feel proficient.

\section{Medical Errors}

Studies have shown that healthcare workers in high demand areas suffer from alarm fatigue which affects their reaction to critical alarms; they silence the audible tone or ignore them altogether (AHRQ, 2005). The noise levels in the hospital environment can also influence the safe and proper use of medical devices (Pape et al, 2005). However, most errors can easily be avoided when healthcare workers are trained to identify potential hazards, circumvent careless mistakes, and follow hospital policies and procedures. Unfortunately it is difficult to identify medical errors during emergencies because the exact cause of death in patients may be a mixture of accidental deaths and deaths due to natural causes (AHRQ, 2005). 


\section{Code errors}

In the Hunt et al., (2009) study 50\% of medical residents never led a code team during CPR. Also, $92 \%$ of the residents recognized apnea and asked the nurse to assist in ventilation with a median time of 24.5 seconds. Seven percent never asked for assistance with ventilation. Sixty six percent of the residents failed to start compressions within one minute of pulselessness, and 33\% never started compressions during the entire scenario. Study results also revealed that $48 \%$ of the residents had at least one unsuccessful attempt at discharging the defibrillator (forgetting to charge the defibrillator), and $83 \%$ made at least one error while operating the defibrillator (turning machine on, removing paddles from machine, changing energy level, charging the defibrillator, and confusion with buttons and cables on the machine) (Hunt et al., 2009).

In the present study, $55 \%$ of the $\mathrm{RN}$ groups (22 out of 40 ) made errors when performing both compressions and ventilations compared to $74 \%$ of medical residents in the Hunt et al., (2009) study. The RN control group (80\%) committed more compression errors than the RN intervention group (30\%). Also there were more ventilation errors committed by the $\mathrm{RN}$ control group (85\%) when compared to the RN intervention group (25\%). However, both groups had the same amount of errors (85\%) for medication administration which demonstrated a deficiency in this task performance measure regardless of role assignment.

More than half (62\%) of the total $\mathrm{RN}$ groups were able to use the defibrillator properly when compared to $83 \%$ of medical residents in Hunt's study. More nurses in the intervention group (75\%) had proficient defibrillation skills compared to the control group (50\%), both groups made errors (poor pad placement, problems turning the machine on, and clearing the team prior to delivering the shock). 


\section{Technical skills}

Technical factors such as code location, scarcity of equipment, and unfamiliar team members may result in total environmental confusion, poor communication between team members, and other organizational factors that may contribute to collateral damage (damage to other patients not involved in the code) (Lipshutz et al., 2008).

The study by DeVita et al, (2005) of individuals (physicians, nurses, and respiratory therapists) as part of a medical emergency response team revealed that the rate of simulated survival increased from zero percent to $90 \%$ for SCA patients. Additionally, the task completion rate improved from $31 \%$ to $89 \%$. Performance in each simulated role (team leader, airway manager, airway assistant, procedure physician, chest compressions, medication/equipment cart, data manager/recorder, and bedside nurse) improved from $10 \%$ to $45 \%$ during the first session and $80 \%$ to $95 \%$ during the third session. The study results also showed that predetermined roles improved task completion associated with that role because most individuals felt comfortable with task assignment. However, if another responder possessed better skills for a certain role then a switch of that person into the role occurred (DeVita et al., 2005).

The results of the present study were similar with the completion of technical skills. Overall $98 \%$ of nurses in both groups either partially or completely performed the tasks for compressions and bag-mask ventilations. However RNs in the intervention group (using color labeled smocks) correctly completed more skills and were significantly faster in their performance for compressions and ventilation than the control group. There were no significant differences between the groups administering medication because $78 \%$ of the total groups of RNs did not give medications. Seventy five percent of the RNs in the intervention group were 
able to demonstrate proficient use of the defibrillator when compared to $50 \%$ in the control group. Results again suggest that pre-assigned visible roles using the smocks and focused assignments (responsible for only one task) can improve CPR performance and potentially patient outcomes.

\section{Team performance Skills}

Interruptions during CPR have been shown to have negative consequences in overall survival and poor patient outcomes (Marsch et al., 2005). Perkins and colleagues noted that after 25 years of training individuals in CPR, retention of cardiopulmonary skills are closely associated with the instructor, learner, curriculum, and frequency of training (i.e. once per year or biannually) (Perkins et al, 2008).

The pilot study by Davis et al., (2008) on the effectiveness of interdisciplinary team training strategies on human factors (the provider, the team, and the hospital system design for safety) and latent conditions (vigilance and surveillance in monitoring) during emergencies revealed lapses in communication, team failures, and latent conditions. Examining failure mode and effect analysis (FMEA) showed that the top three failure modes during emergency C-section were; 1) unclear role definition of team members, 2) inconsistent process for ordering blood products in the operating room, and 3) lack of closed loop communication between the operating room and blood bank (Davis et al.,2008).

In a crossover prospective study, Shapiro et al. (2004) found that the intervention group showed an improvement in team work behaviors when using a specific scoring sheet (Team Dimension Rating Form (TDRF). In the study by Tschan et al., (2011) many unnecessary interruptions were identified in CPR delivery. Their results showed that resuscitation exercises lasted between 2.8 and 7.6 minutes; the team engaged in unnecessary interruptions in $32 \%$ of the 
resuscitation time. Additionally, one group member spent $84 \%$ of the time engaged in a secondary activity increasing unnecessary interruption time for the entire group. In the majority of the unnecessary interruption time individuals focused on the patient monitor or technical aspects of the defibrillator (Tschan et al., 2011).

In the present study team performance focused on various characteristics of the team (problem solving, situational awareness, resource utilization, leadership and communication). Although there were not many differences between the control and the intervention groups for most of these characteristics, the intervention group correctly performed more tasks (prompt assessment, concurrent management, avoid fixation errors, stay calm, and communicate clearly) related to these team performance measures. Also the intervention group (55\%) was more likely to use available resources (call for help and designate tasks) when compared to the control group $(10 \%)$. These findings suggest that highly visible color-labeled smocks with role identification help team members recognize positions and use resources more effectively.

\section{The use of color-labeled smocks during codes}

For three decades, the use of color coding techniques during pediatric emergencies has been shown to be successful. Therefore, incorporating this basic principle (color-labeled smocks) into routine adult emergencies such as codes may provide similar results. Although, gender may play a role in effective compressions, roles for compressions or other physically demanding tasks can be assigned early. These roles can be assigned to male or female nurses who demonstrate proficient skills in these areas.

Several studies suggest that nurses are constantly interrupted or distracted during the course of their shift which predisposes them to making critical mistakes when providing care to patients (Lipshutz et al, 2008, Pape et al., 2005, \& Aiken et al, 2002). Some strategies to reduce 
these distractions and interruptions have been seen when using visible signage (wearing red vest when administering medication or using a do not disturb sign on the door when preparing medications). Other strategies such as improving the nurse to patient ratios have also been considered effective in preventing careless mistakes (Aiken et al., 2002).

The use of color-labeled smocks during emergencies incorporates all of these medical error prevention strategies. The highly visible smocks use different colors to designate each role of the code team thus allowing members on the code team to clearly identify each person's role. These pre-assigned roles also help the nurse and other healthcare professionals focus only on their specific task. The benefits of using color-labeled smocks during resuscitation may help reduce scene chaos, improve technical skill performance, and potentially improve patient outcomes.

\section{Clinical implications}

Medical errors occur 39 times more frequently during emergencies. In hospital settings nurses are the largest group of healthcare workers and may account for nearly $55 \%$ of all medical errors (Lipshutz et al, 2008). To date, few initiatives have shown a reduction in preventable medical errors (Watcher's, 2010). One of the most promising strategies may be the use of colorlabeled smocks.

The simple color-labeled smocks can be incorporated into any code training exercise worldwide. These light weight and inexpensive smocks (aprons) can easily be added to the equipment on any existing crash cart or emergency box. The high quality durable material makes them easy to launder and reuse. Therefore, the clinical implication for implementing an inexpensive color-labeled role assignment tool to improve CPR technical skills among a team of healthcare providers may prove to be a valuable patient safety strategy. 


\section{Study Limitations}

While the original plan was to include 8 RNs per group recruited from UM-JMH, changes in health care delivery resulted in recruiting RNs from other training programs that had a preset number of participants (five to eight member groups versus all eight member groups). To compensate for this the same size groups were balanced in the control and intervention groups. Study findings between groups indicated that this change was not a study limitation.

A potential study limitation was the decision to use only the first five minutes of the code exercise. This may have prevented the RNs from changing roles which would have allowed more team interaction. Only RNs participated in this study of which few had a masters degree but were not APNs'. Results cannot be generalized to physicians or advanced practiced nurses. In these groups improved results for medication administration and defibrillation may have been demonstrated.

\section{Implications for Future Research}

The outcome for SCA survival with CPR in a hospital setting remains low and new strategies aimed at improving outcomes for patients who have had a SCA event are needed. Future studies are needed to evaluate various components of CPR to identify ways to increase knowledge and skill retention. Further research is needed to test the color-labeled smocks in a more heterogenic sample using an interdisciplinary approach. This safety strategy should be tested using a longer duration period (20-30 minutes) of the code event which may represent the actual time used in resuscitation efforts. Finally, the color-labeled smocks should be studied during real codes in a hospital setting to determine their feasibility (ease of use), rate of reducing 
errors, and survival outcomes. Other environmental components such as scene chaos, noise levels, and team work related to CPR using the color-labeled smocks should also be tested Based on the findings of this study and previous studies, additional mock training courses for each person should be required to improve survival. This required training should begin as early as the final semester for graduate nurses during their formal training since the majority of new graduates regardless of education level will start working in a hospital setting. Early exposure to emergency training and team building skills during an emergency is paramount.

\section{Summary}

Although, the 2010 American Heart Association (AHA) basic life support recommended guidelines were improved over previous versions, the rates of survival of victims of sudden cardiac arrest (SCA) using CPR remain low and have shown little improvement over three decades (CDC, 2011). Since RNs comprise the largest group of healthcare providers in U.S. hospitals, (U.S. Labor Bureau, 2010) it is essential that they are competent in performing the four primary measures (compression, ventilation, medication administration, and defibrillation) of CPR in order to improve survival rates of SCA patients. CPR training is needed since many RNs are uncomfortable performing some CPR skills.

One promising teaching and patient safety strategy combines color coding and visible signage of eight roles on the code team in an effort to improve technical skills and team performance. This simple and inexpensive technique can benefit both nurses and patients. Nurses may feel comfortable in performing only one pre-assigned skill during an emergency. Other healthcare providers are able to identify code team members' and their role and not mistake family members or observers as part of the team. 
The use of color-labeled smocks during emergencies incorporates medical error prevention strategies and resulted in shorter mean times to start compressions (63 seconds), and bag mask ventilations (76 seconds). There were a fewer number of errors committed by RNs in the intervention group for both compressions (6) and ventilations (5) when compared to the control groups 16 for compressions and 17 for ventilations. Also, RNs in the intervention group (75\%) completed more technical skills correctly than the control group (50\%). Although, team performance was similar in both groups, RN's in the intervention group (55\%) used more resources (call for help and delegate tasks) appropriately than the control group (10\%). The simple color-labeled smocks can be incorporated into any code training exercise worldwide and potentially improve patient survival from SCA. 


\section{List of References}

Abella, B.S., Alvarado, J.P., Myklebust, H., Edelson, D.P., Barry, A., O’Hearn, N., Vanden Hoek, T.L., \& Becker, L.B. 2005, Quality of Cardiopulmonary Resuscitation During In-Hospital Cardiac Arrest JAMA 293(3):305-310

Abella, B.S. Phil, M., Sandbo, N., assilatos, P., Alvarado, J.P., O’Hearn, N., Wigder, H.N., Hoffman, P., Tynus, K., Vanden Hoek, T.L., Becker, L.B., 2005 Chest Compression Rates during Cardiopulmonary Resuscitation Are Suboptimal A Prospective Study during In-Hospital Cardiac Arrest Circulation111:428-434

Agency for Healthcare Research and Quality (AHRQ) 2004 Hospital nurse staffing and quality of care Issue\#14 Retrieved on September 22, 2009 www.ahrq.org

Aiken, L., Clarke, S., Sloane, D., Sochalski, J., \& Silber, J: 2002 Hospital nurse staffing and patient mortality, nurse burnout, and job dissatisfaction JAMA 288(16) 19871993

American Heart Association guidelines (2010) Highlights of the 2010 American Heart Association guidelines for CPR and ECC. Retrieved March 8, 2011 from http://www.heart.org

Broselow, J. DeBoer, S. Seaver M. 2005 Color Coding to Reduce Errors: The BroselowLuten system streamlines pediatric emergency treatment: AJN, American Journal of Nursing Volume 105: 68 - 71

Buckley, T., and Gordon, C. 2010 The effectiveness of high fidelity simulation on medical-surgical registered nurses ability to recognize and respond to clinical emergencies Nurse Education Today doi:10.1016/j.net.2010.04.004

Centers for Disease and Prevention (CDC), Morbidity Mortality Weekly Report MMWR, 2005, retrieved March 1, 2011 The Centers for Disease Control and Prevention (CDC) National Nosocomial Infections Surveillance (NNIS, 2011)

Clarke, S., \& Aiken, L. 2003 Failure to rescue American Journal of Nursing 103(1) 42-47

DeCarlo, D., Collingridge, D.S. Grant, C., V 2008 Factors influencing nurses' attitude towards simulation based education Simulation in Healthcare 3:90-96

Department of Defense (DoD) and the Department of Veterans Affairs (VA) 2004 Preventing medical errors through electronic reporting Retrieved March 5, 2010

Department of Health and Human Services 2010 Adverse events in hospitals: National incidence among Medicare beneficiaries, Office of Inspector General Retrieved March 5, 2010 
DeVita, M.A., Schaefer, J., Lutz, J., Wang, H., \& Dongilli, T., 2005 Improving medical emergency team (MET) performance using a novel curriculum and a computerized human patient simulator Quality Safety Health care 14:326-331

Florida Center for Nursing 2010 Addressing the Nursing Shortage through Simulation Retrieved February 8, 2010 from http://www.flcenterfornursing.org

Fran, Mary 2010, American Heart Association guidelines (2010) Highlights of the 2010 American Heart Association guidelines for CPR and ECC Retrieved January 19, 2011 from http://www.heart.org

The Food and Drug Administration (FDA) 2000 report Retrieved on February 28, 2010 from http://www.fad.gov

Friedman, S., Provan, D., Moore, S, \& Hanneman, K. (2008) Errors, near misses and adverse events in the emergency department: What can patients tell us? Canadian Journal of Emergency Medicine 10 (5) 421-427

Hawkins JL. (2003) Anesthesia-related maternal mortality Clinical Obstetrics Gynecology 46:679-87

Henneman, P., Fisher, D., Henneman, E., Pham, T., Campbell, M., \& Nathanson, B. (2009) Patient identification errors are common in a simulated environment Annuals of emergency medicine 55(6)503-509

Hitcho, E.B., Krauss, M. J., Birge, S., Dunagan, W.C., Fraser V.J., Fischer, I., Johnson, S., Nast, P.A. \& Costantinou, E., (2004) Characteristics and Circumstances of Falls in a Hospital Setting A Prospective Analysis J GEN INTERN MED 19:732-739

Hohenhaus S.M. 2002 Assessing competency: The Broselow-Lueten resuscitation tape Journal of Emergency Nurses 2(1):70-2.

Hove, L.D., Nielsen, H.B., \& Christoffersen, J.K., (2006) Patient injuries in response to anesthetic procedures: cases evaluated by the Danish Patient Insurance Association Acta Anesthesiology Scandinavia 2006; 50: 530 — 535

Hunt, E.A., Verad, K., Diener-West M., Haggerty, J.A., Nelson, K.H., Shaffner, D.H., \& Pronovost P.J. 2009, Delays and errors in cardiopulmonary resuscitation and defibrillation by pediatric residents during simulated cardiopulmonary arrests Resuscitation 80:819-825

International Liaison committee on resuscitation (ILCOR) 2010 ERC and AHA guidelines retrieved on January 15, 2010 from http://www.ilcor.org/en/consensus

Institute for Healthcare Improvement (IHI) (2008) report American academy of physician assistants report, 2006 retrieved on November 2, 2010 100K Lives 
Campaignwww.ihi.org/IHI/Programs/Campaign

Issenberg, S.B. \& Scalese, R. (2007) Simulation in health care education Perspectives in Biology and Medicine 51:31-36

Joint Commission Accreditation Hospitals and organizations (JACHO) 2007

Sentinel events www.jointcommission.org

http://www.jointcommision.org/PatientSafety/UniversalProtocol

Kaushual, R., Shojania, K., \& Bates, D. 2003 Effects of computerized physician Order entry and clinical decision support systems on medication safety Archives of Internal Medicine 163:1409-1416

Kim J., Neilipovitz, D., Cardinal P., \& Chiu M., 2009: A Comparison of Global Rating Scale and Checklist Scores in the Validation of an Evaluation Tool to Assess Performance in the Resuscitation of Critically Ill Patients During Simulated Emergencies (Abbreviated as "CRM Simulator Study IB Simulation in Healthcare 10:1097-1103

Kohn, L.T., Corrigan, J.M., \& Donaldson, M.S. (2000) To Err is Human: Building a Safer health system Committee on Quality of Health Care in America: Institute of Medicine National Academy Press 2:24-37

Kozer, E., Seto, W., Verjee, Z., Parshurum, C., Khattak, S., Koren, G., \& Jarvis, A (2004) Prospective observational study on the incidence of medication errors during simulated resuscitation in a pediatric emergency department BMJ DOI 10.1136/bmj.38244.607083.55

Kozer, E., Scolnik, D., Macpherson, A., Keays, T., Shi, K., Luk T., \& Koren G., (2002) Variables Associated With Medication Errors in Pediatric Emergency Medicine Pediatrics 110:737-742

Kwaan, M.R., Studdert, D.M., Zinner, M.J. \& Gawande, A.A., (2006) Incidence, Patterns, and Prevention of Wrong-Site Surgery Arch Surg. 141:353-358

Lee B, Gausche-Hill M. Advances in pediatric resuscitation: pediatric airway management. Clin Pediatr Emerg Med. 2001;2(2):91-106

Lipshutz, A., Morlock, L., Shore, A., Hicks, R., Dy, Pronovost, \& Winters, B., (2008) Medications errors associated with code situations in us hospitals: Direct and collateral damage The Joint Commission Journal on Quality and Patient Safety 34(1) 46-56

Lucero, R.J., Lake, E.T., \& Aiken, L.H., 2009 Variations in Nursing Care Quality across Hospitals Advance Nursing. 65(11): 2299-2310

Luten R, Broselow J. Rainbow care: the Broselow-Luten system. Implications for 
pediatric patient safety. Ambulatory outreach. 14-16 October 1999

Luten R, Wears L, Broselow J, Croskerry P, Joseph M, Frush K. Managing the unique size-related issues of pediatric resuscitation: reducing cognitive load with resuscitation aids. Acad Emerg Med. 2002;9(8):840-847

Middleton, K.R., \& Burt, C.W., 2006 (CDC) Advance data from vital health statistics Availability of Pediatric Services and Equipment in Emergency Departments: United States, 2002-03 Number 367 February, 2008.

Miller, K.K., Riley, W., Davis, S., \& Hansen, H.E., 2008 In Situ Simulation A Method of Experiential Learning to Promote Safety and Team Behavior $J$ Perinat Neonat Nurs (22) 2, pp. 105- 11

Needleman, J., Bauerhaus, P.H., Mattke, S., Stuart, M., \& Zelevinsky, Z., 2002

Nurse-staffing levels and the quality of care in hospitals N Engl J Med (346) 22

O'Connor, R.E., Edelson, D., \& Nadkarni, V., 2009 Multi-National Attitudes Among Healthcare Professionals Concerning CPR, Ipsos Health 11(09)

Pape, T.M. Guerra, D,M., Muzquiz, M., Bryant, J.B., Ingram, M., Schanner, B., Alcala, A., Sharp, J., Bishop,D., Carreno, E., \& Welker, J., 2005 Innovative approaches to reducing nurses' distractions during medication administration The journal of continuing education in nursing 36(3) 108-116

Perkins, G.D., Boyle, W., Bridgestock, H., Davies, S., Oliver, Z., Bradburn, S., Green, C., Davies, R., \& Cooke, M.W., 2008 Resuscitation 77:69-74

Porter, S.C. Kaushal, R., Forbes, P., Goldmann, R., \& Kalish, L.A., 2008 Impact of a Patient-Centered Technology on Medication Errors During Pediatric Emergency Care Ambulatory Pediatrics 8:329-35

Reasons, J. 2000 Human errors: models and management BMJ 320: 768-770

Seropian, M.A., 2003 General concepts in full scale simulation: Getting started Anesthesia \& Analgesia 97:16995-1705

Scott, L.D. Rogers, A.E., Hwang W.T., and Zhang Y., 2006 Safety Effects of Critical Care Nurses' Work Hours on Vigilance and Patients' Am J Crit Care 15: 30-37

Sharek, P.J., Parast, L., Leong, K., Coombs, J., Earnest, K., Sullivan, J., Frankel, L., \& Roth, J.: 2007 Effect of a Rapid Response Team on Hospital-wide Mortality and Code Rates Outside the ICU in a Children's Hospital JAMA.298 (19):2267-2274. 
Stelfox, H., Palmisani, S., Scurlock. C., Orav, E., \& Bates, D., (2006) the "To err is Human" report and the patient safety literature Quality and Safety Healthcare 15:174178

Steurer, L.M., \& Luhmann J., 2007 Adverse Effects of Pediatric Emergency Sedation after Discharge Pediatric Nursing 33(5)403-407

Tang, F- I., Sheu, S- J., Yu S., Wiel, I-L., \& Chen, C-H., (2007) Nurses relate the contributing factors involved in medication errors Journal of Clinical Nursing 16, 447-457

Taylor ,B., Marcantonio, E., Pagovich, O., Carbo, A., Bergmann, M., Davis, R., (2008) Do Medical Inpatients Who Report Poor Service Quality Experience More Adverse Events and Medical Errors? Med Care 46 (2): 224-228

Vasileff, H., Whitten, L., Pink, J., Goldsworthy, S., \& Angley, M. 2009 The effects of medication errors of pharmacists charting medication in an emergency department Pharmacy World Science 10(31) 373-379

Vilke G, Marino A, Fisher R, Chan T. Estimation of pediatric patient weight by EMT-Ps. J Emerg Med. 2001;21(2):125-128

Wachter, R.M. 2010 Patient Safety at Ten: Unmistakable progress, troubling gaps Health Affairs 29(1) 165-173 


\section{APPENDICES}

\section{Appendix A CPR Scenario Technical Skills Score Sheet}

\begin{tabular}{|c|c|c|}
\hline \multirow{2}{*}{$\begin{array}{l}0=\text { no attempt } \\
1=\text { incompletely performed } \\
2=\text { completely performed } \\
\begin{array}{l}\text { Basic Life Support (BLS) } \\
\text { (Mock Code Blue) }\end{array}\end{array}$} & \multirow[t]{2}{*}{ Score } & \multirow[t]{2}{*}{ Comment } \\
\hline & & \\
\hline \multicolumn{3}{|l|}{ Patient Scenario (if pt awake) } \\
\hline \multirow{2}{*}{\multicolumn{3}{|c|}{$\begin{array}{l}\text { Wash hands } \\
\text { Introduce self }\end{array}$}} \\
\hline & & \\
\hline \multicolumn{3}{|l|}{\begin{tabular}{|l}
$\begin{array}{l}\text { Identify patient (using two pt } \\
\text { identifiers) }\end{array}$
\end{tabular}} \\
\hline \multirow{2}{*}{\multicolumn{3}{|c|}{ Chief complaint }} \\
\hline Obtain past medical history & & \\
\hline \multicolumn{3}{|l|}{ Identify allergies } \\
\hline \multicolumn{3}{|l|}{ Obtain vitals } \\
\hline \multicolumn{3}{|l|}{ Initial patient assessment } \\
\hline \multicolumn{3}{|l|}{$\begin{array}{l}\text { Establish level of } \\
\text { consciousness }\end{array}$} \\
\hline \multicolumn{3}{|l|}{$\begin{array}{l}\text { Call for help/ask for AED or } \\
\text { defibrillator } \\
\text { Call Physician } \\
\text { Activate the Rapid Response } \\
\text { Team } \\
\text { Call a Code Blue }\end{array}$} \\
\hline \multicolumn{3}{|l|}{$\begin{array}{l}\text { Airway (Head-tilt chin lift or } \\
\text { Jaw Thrust if trauma is } \\
\text { suspected, patent airway) }\end{array}$} \\
\hline \multicolumn{3}{|l|}{$\begin{array}{l}\text { Breathing } \\
\text { (assess breathing pattern) }\end{array}$} \\
\hline \multicolumn{3}{|l|}{ Circulation (check pulse) } \\
\hline \multicolumn{3}{|l|}{$\begin{array}{l}\text { Compressions (check } \\
\text { compression rate and depth) }\end{array}$} \\
\hline \multicolumn{3}{|l|}{\begin{tabular}{|l}
$\begin{array}{l}\text { Advance Life Support (ALS) } \\
\text { (Mock Code Blue) }\end{array}$ \\
\end{tabular}} \\
\hline \multicolumn{3}{|l|}{ Identify role of team members } \\
\hline \multicolumn{3}{|l|}{$\begin{array}{l}\text { Maintain patent airway and } \\
\text { ventilate as needed }\end{array}$} \\
\hline \multicolumn{3}{|l|}{$\begin{array}{l}\text { Assess circulation (Pulse/BP) } \\
\text { monitor/manual }\end{array}$} \\
\hline \multicolumn{3}{|l|}{$\begin{array}{l}\text { Chest compressions (proper } \\
\text { ratio and depth) }\end{array}$} \\
\hline \multicolumn{3}{|l|}{ Identify abnormal rhythms } \\
\hline Proper use of defibrillator & & \\
\hline
\end{tabular}




\begin{tabular}{|l|l|l|}
\hline $\begin{array}{l}\text { (clear three times before } \\
\text { administering shock and use } \\
\text { proper joules) }\end{array}$ & & \\
\hline Cardioversion as indicated & & \\
\hline $\begin{array}{l}\text { Use and apply Pacer as } \\
\text { indicated }\end{array}$ & & \\
\hline $\begin{array}{l}\text { Administer Medication } \\
\text { According to Cardiac Rhythm }\end{array}$ & & \\
\hline Epinephrine & & \\
\hline Vasopressin & & \\
\hline Atropine & & \\
\hline Amiodarone & & \\
\hline Lidocaine & & \\
\hline Other medications & & \\
\hline Magnesium & & \\
\hline Adenosine & & \\
\hline Beta Blocker & & \\
\hline Calcium Channel Blocker & & \\
\hline Dopamine & & \\
\hline Epinephrine Drip & \\
\hline Patient Outcome & \\
\hline Patient survival & \\
\hline Patient transfer to ICU & \\
\hline Total Score & \\
\hline 0=no attempt & \\
\hline 2=incompletely performed & & \\
\hline
\end{tabular}


Appendix B

CPR Scenario Time and Medical Error Checklist

\begin{tabular}{|c|c|c|}
\hline $\begin{array}{l}0=\text { no attempt/incomplete } \\
1=\text { completely performed } \\
2=\text { completely performed }\end{array}$ & Time & Type of Medical Error \\
\hline $\begin{array}{l}\text { Basic Life Support (BLS) } \\
\text { (Mock Code Blue) }\end{array}$ & & \\
\hline Patient Scenario (if pt awake) & & \\
\hline Wash hands & & \\
\hline Introduce self & & \\
\hline $\begin{array}{l}\text { Identify patient (using two pt } \\
\text { identifiers) }\end{array}$ & & \\
\hline Chief complaint & & \\
\hline Obtain past medical history & & \\
\hline Identify allergies & & \\
\hline Obtain vitals & & \\
\hline Initial patient assessment & & \\
\hline $\begin{array}{l}\text { Establish level of } \\
\text { consciousness }\end{array}$ & & \\
\hline $\begin{array}{l}\text { Call for help/ask for AED or } \\
\text { defibrillator } \\
\text { Call Physician } \\
\text { Activate the Rapid Response } \\
\text { Team } \\
\text { Call a Code Blue }\end{array}$ & & \\
\hline $\begin{array}{l}\text { Airway (Head-tilt chin lift or } \\
\text { Jaw Thrust if trauma is } \\
\text { suspected, patent airway) }\end{array}$ & & \\
\hline $\begin{array}{l}\text { Breathing } \\
\text { (assess breathing pattern) }\end{array}$ & & \\
\hline Circulation (check pulse) & & \\
\hline $\begin{array}{l}\text { Compressions (check } \\
\text { compression rate and depth) }\end{array}$ & & \\
\hline $\begin{array}{l}\text { Advance Life Support (ALS) } \\
\text { (Mock Code Blue) }\end{array}$ & & \\
\hline Identify role of team members & & \\
\hline $\begin{array}{l}\text { Maintain patent airway and } \\
\text { ventilate as needed }\end{array}$ & & \\
\hline $\begin{array}{l}\text { Assess circulation (Pulse/BP) } \\
\text { monitor/manual }\end{array}$ & & \\
\hline $\begin{array}{l}\text { Chest compressions (proper } \\
\text { ratio and depth) }\end{array}$ & & \\
\hline Identify abnormal rhythms & & \\
\hline Proper use of defibrillator & & \\
\hline
\end{tabular}




\begin{tabular}{|l|l|l|}
\hline $\begin{array}{l}\text { (clear three times before } \\
\text { administering shock and use } \\
\text { proper joules) }\end{array}$ & & \\
\hline Cardioversion as indicated & & \\
\hline $\begin{array}{l}\text { Use and apply Pacer as } \\
\text { indicated }\end{array}$ & & \\
\hline $\begin{array}{l}\text { Administer Medication } \\
\text { According to Cardiac Rhythm }\end{array}$ & & \\
\hline Epinephrine & & \\
\hline Vasopressin & & \\
\hline Atropine & & \\
\hline Amiodarone & & \\
\hline Lidocaine & & \\
\hline Other medications & & \\
\hline Magnesium & & \\
\hline Adenosine & & \\
\hline Beta Blocker & & \\
\hline Calcium Channel Blocker & & \\
\hline Dopamine & & \\
\hline Epinephrine Drip & \\
\hline Patient Outcome & \\
\hline Patient survival & \\
\hline Patient transfer to ICU & & \\
\hline Total Score & & \\
\hline 0=no attempt/incomplete \\
2=completely performed
\end{tabular}


Appendix C

Ottawa CRM checklist

\begin{tabular}{|l|l|l|l|}
\hline Action & Yes (2 points) & With prompting (1 point) & No (0 points) \\
\hline PROBLEM SOLVING & & & \\
\hline Prompt ABC assessment & & & \\
\hline $\begin{array}{l}\text { Implements concurrent } \\
\text { management approaches }\end{array}$ & & & \\
\hline SITUATIONAL AWARENESS & & & \\
\hline Avoids fixation error & & & \\
\hline $\begin{array}{l}\text { Re-assesses and re-evaluates } \\
\text { situation }\end{array}$ & & & \\
\hline RESOURCE UTILIZATION & & & \\
\hline Calls for help when indicated & & & \\
\hline Delegates and directs appropriately & & & \\
\hline LEADERSHIP & & & \\
\hline Maintains calm demeanor & & & \\
\hline $\begin{array}{l}\text { Acts decisively and maintains } \\
\text { control of crisis }\end{array}$ & & & \\
\hline Maintains global perspective & & & \\
\hline COMMUNICATION & & & \\
\hline $\begin{array}{l}\text { Communicates clearly and } \\
\text { concisely }\end{array}$ & & & \\
\hline Close loop and uses names & & & \\
\hline Listens to input & & & \\
\hline Total score (points) & & & \\
\hline $\begin{array}{l}\text { Participant number } \\
\text { Scorer number }\end{array}$ & & & \\
\hline
\end{tabular}




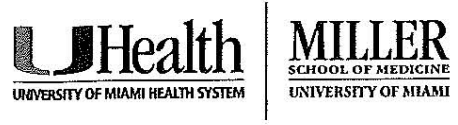

\section{Jackson 雷畻}

January 5, 2012

Letter of Access

To Whom It May Concern:

This letter is to state that Ruth Thomas has access to the University of Miami-Jackson Memorial Hospital Center for Patient Safety (CPS) to complete her research project for dissertation. Ruth is currently employed at the Center for Patient Safety and assists nurses with various simulation projects to assure a component of patient safety is included in all training sessions performed at the Center. Ruth's research project to test a SMOCK system on CPR primary measures and medical errors during simulated emergencies is aligned with the Center's mission and vision to build safer healthcare.

The Center for Patient Safety opened a state-of-the-art simulation facility in 2004, which consists of a control room, an operating room, an emergency room/ICU, exam rooms, and an exact replica of a University of Miani Hospital room to evaluate the impact of design changes on patient safety, a conference room for didactic teaching and debriefings, and a micro-simulation laboratory.

The simulation area was designed to accommodate single and multi-patient simulations and recreate various patient care areas. To suspend disbelief, all aspects of actual treatment areas, including surgical and anesthesia equipment, medications, medical gases, overhead lights, and x-ray film illuminators, have been incorporated in the simulation space. The core of the simulation area is an array of computercontrolled, full-body patient simulators that talk, present realistic vital signs, and respond in real time to clinical interventions. The control room contains the core of the audio-visual system with full monitoring and recording capabilities.

The Center for Patient Safety has the resources for Ruth to perform and complete her research project.

Sincerely,

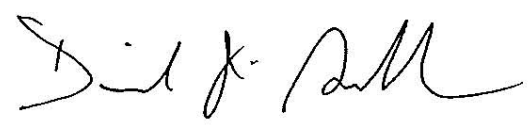

David J. Birnbach, M.D., M.P.H.

Professor, Department of Anesthesiology

Senior Associate Dean for Safety, Quality and Risk Prevention

Director, UM-JMH Center for Patient Safety.

Miller School of Medicine

Vice Provost, University of Miami

UM-JMH Center for Patient Safety

Jackson Memorial Hospital

1611 Northwest 12th Avenue, Institute, 4th Floor | Miami, Florida 33136

Phone: (305) 585-8364 | Fax: (305) 585-1475 


$\begin{array}{ll}\text { From: } & \text { John Kim [jkim@aserty.com] } \\ \text { Sent: } & \text { Tuesday, June 05, 2012 11:09 PM } \\ \text { To: } & \text { Everett-Thomas, Ruth } \\ \text { Subject: } & \text { Re: Permission to use the GRM checklist }\end{array}$

Hi Ruth,

Thanks for your interest in the Ottawa CRM checklist - you have my permission to use the Ottawa CRM checklist, so long as its use is recognized in any manuscript/presentation.

One additional comment - you referred to using the checklist for determining if certain procedures were done. The checklist provides for key CRM actions to be done - but you may wish to provide specific actions to each case to help increase improve the scoring accuracy of your team (I.E. Delegating specific tasks particular to that case <not simply "delegates tasks appropriately">, anticipating likely events such as hypotension / need for intubation <instead of "anticipates likely events">). Once you do this, the checklist is only applicable to that case - but it often improves the scoring accuracy significantly. Doing a trial run and going through the rater training process will help as well, but I thought the suggestion to tailor the checklist to your cases might prove helpful.

Best of luck,

John Kim, MD, M.Ed, FRCPC

Associate Professor, Department of Critical Care Medicine

University of Ottawa/ The Ottawa Hospital

Primary Author

CRM Simulator Study I

From: "Everett-Thomas, Ruth"< <REverett@med.miami.edu $>$

Date: Tuesday, June 5, 2012 3:46 PM

To: John Kim<jkim@aserty.com>

Cc: John Work<jkim@ottawahospital.on.ca>

Subject: Permission to use the GRM checklist

Good afternoon Dr. Kim my name is Ruth Thomas and I currently work as a nurse at the University of Miami-Jackson Memorial Hospital Center for Patient Safety which houses a simulation Center. Our sim Center is hospital based and we are responsible for training our house staff of nurses and physicians from all departments.

I am also pursuing a PhD in nursing and using simulation to identify errors or patient safety concerns during nurse training as well as team training performances among nurses during a crises(specifically mock codes). I read your article "A Comparison of Global Rating Scale and Checklist Scores in the Validation of an Evaluation Tool to Assess Performance in the Resuscitation of Critically III Patients During Simulated Emergencies (Abbreviated as "CRM Simulator Study IB")", and I would like to use your checklist tool as part of my project to analyze nurse team performance during simulation training of Mock codes.

I prefer using the checklist versus the global rating scale because I am looking only to determine if a procedure during the code was performed or not. Therefore, I would like your permission to use this tool to analyze the data collected during my study, and all of the sessions will be videotaped.

Thank you in advance for your consideration, 


\title{
Appendix G $\quad$ FIU-IRB Approval Letter
}

\section{뭄Iㅁ FLORIDA INTERNATIONAL UNIVERSITY}

Office of Research Integrity Research Compliance, MARC 270

\section{MEMORANDUM}

\author{
To: Ruth Thomas \\ CC: $\quad$ Dr. Dorothy Brooten \\ File \\ From: \\ Jada Dixon, MJ, MPH, IRB Coordinator $\varnothing$ \\ Date: $\quad$ February 8, 2012 \\ Proposal Title: Test of a SMOCK System on CPR Primary Measures and Medical Errors During \\ Simulated Emergencies
}

IRB Exemption \# 011912-00

The Social and Behavioral Institutional Review Board of Florida International University has reviewed your study for the use of human subjects via the Exempt Review process. Your study was deemed Exempt by the Institutional Review Board on January 19, 2012.

As a requirement of IRB approval you are required to:

1) Submit a completion report (Form B-2) upon completion of your project in order for the file to be closed.

2) Submit a proposal and receive approval for any additions or changes in the procedures involving human subjects.

3) Provide immediate written notification to the IRB of every serious or unusual or unanticipated adverse event as well as problems with the rights or welfare of the human subjects. You must confirm the receipt of serious AE reports with the IRB office.

4) Close out your project with the IRB office when the study is finished or discontinued.

Special Conditions: -Upon approval from the University of Miami IRB, a copy of the Unversity of Miami approval letter must be submitted to the FIU IRB Office (Office of Intergrity) to complete process..

Please note that your IRB exemption number is indicated above. For further information, you may visit the FIU IRB website at http://research,fiu.edu/compliance/compliance.html. 
Study \#:

DATE:

Please provide the following demographic information:

Age: Gender: Male $\quad$ Female

Degree

Area of hospital where you work Number of years work in Nursing/Healthcare

Last date CPR recertification do not remember

Last time you participated in a mock code

How many times have you participated in a mock code in the past?
$\square 0$
$\square 1-5$
$\square 6-10$
$\square 11-15$
$\square 16+$

How many times have you participated in a real life code in the past?
$\square 0$
$\square 1-5$
$\square$ 6-10
$\square 11-15$
$\square 16+$

How many times have you participated in a real life code in an out of hospital setting in the past?
$\square 0$
$\square 1-5$
$\square$ 6-10
11-15
$\square 16+$

Do you feel that you have adequate skills in CPR performance measures? $\square$ Yes $\quad \square$ No What are your strengths in CPR technical skills?

$\square$ Ventilation $\quad \square$ compressions $\quad \square$ medication administration $\quad \square$ defibrillation

Explain

What are your weaknesses in CPR technical skills?

$\square$ Ventilation $\quad \square$ compressions $\quad \square$ medication administration $\quad \square$ defibrillation

Explain

How CONFIDENT do you feel presently in performing CPR?

$\begin{array}{ccccc}\text { Not confident at all } & \begin{array}{c}\text { Average } \\ \text { confidence }\end{array} & \text { Very confiden } \\ \square \mathbf{1} & \square \mathbf{2} & \square \mathbf{3} & \square \mathbf{4} & \square 5\end{array}$

How COMPETENT do you feel presently in performing CPR?

Need additional Instruction
$\square 1$

$\square 2$
Need a little more practice

$\square \mathbf{3}$
No further instruction needed 
Appendix I

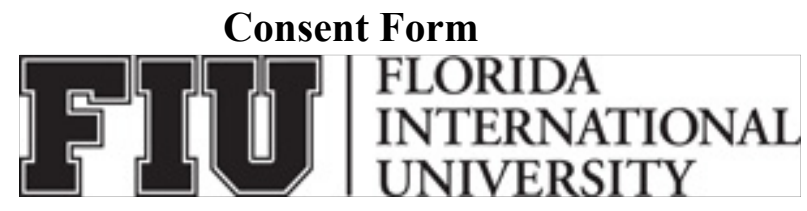

\author{
ADULT CONSENT TO PARTICIPATE IN A RESEARCH STUDY \\ Test of a SMOCK system on CPR primary measures and medical errors during simulated \\ emergencies
}

P.I. Ruth Everett-Thomas RN, MSN, PhDc

Department: Anesthesiology: Center for Patient Safety

Phone: 305 585-8364, Direct line: 305 585-1465

Email address: reverett@med.miami.edu or rthom005@fiu.edu

\title{
PURPOSE OF THE STUDY
}

You are being asked to be in a research study. The purpose of this study is to evaluate CPR primary measures during simulation training (mock codes). You are being asked to be in a study as an integral part of nurse training using simulation as a tool to enhance formal didactic education.

\section{NUMBER OF STUDY PARTICIPANTS}

If you decide to be in this study, you will be one of 320 people in this research study.

\section{DURATION OF THE STUDY}

Your participation will require participating in a 6 minute clinical simulated CPR scenario followed by 12 minutes of debriefing/review of your critical thinking and technical skills. All sessions will be video recorded and scored according to the basic life support or advance cardiac life support (BLS/ACLS) recommendations.

\section{PROCEDURES}

If you agree to be in the study, we will ask you to do the following things:

You may participate in several simulation scenarios as part of your internship or CPR recertification training program. For this study we will only use one experience preferably your first session. Your medical knowledge related to various components of CPR and technical skills will be assessed through simulation exercises.

All exercises will take place in simulation center, and as part of the training all participants will receive feedback during the debriefing portion of the study. Each simulation session will consist of a different scenario and the level of complexity may vary from one scenario to the next. All of the training exercises will be performed as a group and technical skills and team performance will be evaluated using the recent 2010 AHA recommendation for BLS/ACLS.

A member from the research team will assess your medical knowledge using an evaluation tool by direct observation (through a one way mirror) or by review of the video post training. All data captured for this study will be de-identified and used in aggregate form for publication and or 
course evaluation.

As a part of your participation, you will be asked to complete a confidential evaluation before and after the training exercise in order for the instructor and research team to improve the training program. This evaluation will also have questions to ascertain information on demographic data and previous simulation and CPR experience.

Signing this document alone does not constitute your permission to use your tape or videotape you, you will be asked to sign an Authorization for Audio/Video/Photography Recording in a Research Study form which allows us to make audio and video recordings.

\section{RISKS AND/OR DISCOMFORTS}

We do not anticipate you will experience any personal risk or discomfort from taking part in this study. Although, your faces will be seen on the video there will be no personal data linking the video to you and all efforts will be made to use these videos as part of this research study only. The information gained from the videos will be de-identified and collected in aggregate form, and no individual data will be collected, identified, or shared with your employer or instructor.

\section{BENEFITS}

The following benefits may be associated with your participation in this study: It is possible that you will benefit from this study by receiving feedback. We hope to impart technical skills and knowledge that can facilitate your efficiency and expertise when performing CPR procedures. Additionally, your comfort level may increase. The results of this study may benefit society by providing additional information that may improve nurse's technical skills when performing CPR.

\section{ALTERNATIVES}

You have the alternative not to participate in this study. You can decide to stop participating in this study at any time.

\section{COSTS}

You will not have any costs associated with your participation in this study.

\section{INCENTIVES/PAYMENTS TO PARTICIPANTS}

There are no payments or other forms of compensation associated with your participation in this study.

\section{COMPENSATION FOR STUDY-RELATED INJURY}

Although injuries are unlikely, if injury should occur, treatment will, in most cases, be available. If you have insurance, your insurance company may or may not pay for these costs. If you do not have insurance, or if your insurance company refuses to pay, you will be expected to pay. Funds to compensate for pain, expenses, lost wages and other damages caused by injury are not available.

\section{Student Rights}

If you are a student, your desire not to participate, or your request to withdraw from the study, will not affect your grades or other academic standings within the University. 


\section{Employee Rights}

If you are an employee of the University, your decision to participate in or to withdraw from the study will not affect your employment within the University.

\section{CONFIDENTIALITY}

The records of this study will be kept private and will be protected to the fullest extent provided by law. In any sort of report we might publish, we will not include any information that will make it possible to identify a subject. Research records will be stored securely and only the researcher team will have access to the records. However, your records may be reviewed for audit purposes by authorized University or other agents who will be bound by the same provisions of confidentiality.

The U.S. Department of Health and Human Services (DHHS) may request to review and obtain copies of your records. The Food and Drug Administration (FDA) may request to review and obtain copies of your records.

A description of this clinical trial will be available on http://www.ClinicalTrials.gov, as required by US Law. This web site will not include information that can identify you. At most, the web site will include a summary of the results. You can search this website at anytime.

If you have any questions relating to your rights as a research subject, please contact the University of Miami's HUMAN SUBJECTS RESEARCH OFFICE (HSRO), at 305-243$\underline{3195}$.

\section{RESEARCHER CONTACT INFORMATION}

If you have any questions about the purpose, procedures, or any other issues relating to this research study you may contact Ruth Thomas at FIU ACH2, research office, 305 348-1689, rthom005@fiu.edu or305 585-1465 reverett@med.miami.edu

\section{PARTICIPANT AGREEMENT}

I have read the information in this consent form and agree to participate in this study. I have had a chance to ask any questions I have about this study, and they have been answered for me. I understand that I am entitled to a copy of this form after it has been read and signed.

Signature of Participant

Printed Name of Participant

Signature of Person Obtaining Consent
Date 


\section{Appendix J UM-IRB Approval Letter}

\section{UNIVERSITY}

OF MIAMI

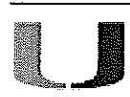

University of Miami

Ph: $305-243-3195$

Human Subjects Research Office (MBOO) Fax. 305-243-3328

PO $80 \times 016900$, Miami, Florida 33101 iwrithsro.mantequ

1500 NW i2 Avenua, Suite 1002, Miami, Florida 33136

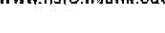

\section{EXEMPT - CONFIRMATION}

March 6, 2012

Ruth Everett, MSN, RN

University of Miami

Department of Anesthesiology

Medical Campus, Locator Code: R-370

Institute, Room $436 \mathrm{~A}$

Miami, FL 33136

HSRO STUDY

NUMBER:

20110864

STUDY TITLE: A retrospective study using video recordings to identify deviations/errors in the 2005 and 2010 AHA recommendations on CPR performance measures during hospital-based simulation training exercises among nurses and physicians

IRB ACTION

DATE:

$12 / 10 / 2011$

FWA \#:

FWA00002247

On December 10, 2011, an IRB Designee determined that the above referenced protocol qualifies for exemption from IRB review, with a waiver of informed consent and full waiver of HIPAA authorization.

\section{APPROVAL INCLUDES:}

New Research Protocol

Research Materials (English Version Only)

- Data Collection Sheet-Crisis Management Skills

- Data collection Sheet-Technical Skills Score Sheet

NOTE: Translations of IRB approved study documents, including informed consent documents, into languages other than English must be submitted to HSRO for approval prior to use.

You must prepare and submit to the Office of HIPAA Privacy \& Security a record of disclosure for each disclosure of patient information under a waiver of authorization by using the HIPAA Accounting for Disclosures form (HIPAA Attachment 45) located on the HSRO HIPAA page.

A request to continue this study must be submitted to the HSRO at least $\mathbf{4 5}$ days before IRB approval expires. If

https:/eprost.med.miami.edu/Eprost/Doc/0/N9Q9N1P8L66KBBELULKGQ16G57/from... 11/13/2012 
this study does not receive continuing IRB approval prior to expiration, all research activities must cease, and it may be officially suspended or terminated.

Please remember that the Human Subjects Research Office (HSRO) must be notified of any proposed changes in research activities. Changes must receive IRB review and approval prior to implementation. Upon completion of the study, submit a closure report via eProst.

Sincerely,

[This is a representation of an electronic record that was signed electronically and this page is the manifestation of the electronic signature]

Amanda Coltes-Rojas, MPH, CIP

Director

Regulatory Affairs \& Educational Initiatives

$/ \mathrm{pn}$

cc: IRB File

Jill Sanko

https://eprost.med.miami.edu/Eprost/Doc/0/N9Q9N1P8L66KBBELULKGQ16G57/from... 11/13/2012 


\section{Appendix k UM-IRB Approval Letter-2}

\begin{tabular}{|c|c|c|}
\hline $\begin{array}{l}\text { UNIVERSITY } \\
\text { OF MIAMI }\end{array}$ & & \\
\hline & 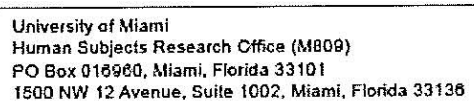 & 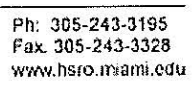 \\
\hline
\end{tabular}

EXPEDITED - APPROVED AS MODIFIED

May 14,2012

Ruth Everett, MSN, RN

University of Miami

Department of Anesthesiology

Medical Campus, Locator Code: R-370

Institute, Room $436 \mathrm{~A}$

Miami, FL 33136

$\begin{array}{ll}\text { HSRO STUDY NUMBER: } & \mathbf{2 0 1 1 0 8 6 4} \\ \text { STUDY TITLE: } & \begin{array}{l}\text { A retrospective study using video recordings to identify deviations/errors } \\ \text { in the } 2005 \text { and } 2010 \text { AHA recommendations on CPR performance } \\ \text { measures during hospital-based simulation training exercises among } \\ \text { nurses and physicians }\end{array} \\ & \mathbf{5 / 1 2 / 2 0 1 2} \\ \text { IRB ACTION DATE: } & \mathbf{5 / 1 1 / 2 0 1 3} \\ \text { STUDY APPROVAL EXPIRES } & \text { FWA00002247 } \\ \text { FWA \#: } & \text { Not Applicable }\end{array}$

On May 12, 2012, an IRB Designee approved the following items under the expedited review process.

\section{APPROVAL INCLUDES:}

Amendment ( Version \# $20110864-01$ ) (Version Date 3/13/2012)

- Change protocol from exempt to expedited review

- Add prospective component to the study-Affected sections of eProst include 4.1, 4.2, 4.3, 4.4, 4.7, 4.8, 4.9, 5.1, 5.2.B, 5.5, 5.7, 6.1, 6.1.C 6.2, 6.3, 6.8, 7.9 and 7.15

- Submit informed consent form and CPR Questionnaire

Research Materials (English Version Only)

- UM Main Informed Consent Form

- Video Consent Form

- CPR Training Questionnaire

MODIFICATIONS INCLUDE: (Please see History Log for document with track changes)

https://eprost.med.miami.edu/Eprost/Doc/0/8LBAHMVML69KL5LR48R4ECC1CE/fro... 11/13/2012 
- Research Materials (English Version Only)

- UM Main Informed Consent Form

This study must be conducted in accordance with IRB approval and you must use the documents as modified by the IRB. If you

do not accept the changes made by the IRB, the study must not be (initiated or continued). If the changes are not acceptable,

you may withdraw the study or appeal to the IRB. Should you have any questions, please contact the HSRO.

NOTE: Translations of IRB approved study documents, including informed consent documents, into languages other than English must be submitted to HSRO for approval prior to use.

A request to continue this study must be submitted to the HSRO at least $\mathbf{4 5}$ days before IRB approval expires. If this study does not receive continuing IRB approval prior to expiration, all research activities must cease, and it may be officially suspended or terminated.

Sincerely,

[This is a representation of an electronic record that was signed electronically and this page is

the manifestation of the electronic signature]

Amanda Coltes-Rojas, MPH, CIP

Director

Regulatory Affairs \& Educational Initiatives

$/ \mathrm{pn}$

cc: IRB file

Jill Sanko

https://eprost.med.miami.edu/Eprost/Doc/0/8LBAHMVML69KL5LR48R4ECC1CE/fro... 11/13/2012 
VITA

\section{RUTH THOMAS}

July 6

Born, Miami Florida

1984 - 1987 Associate of Science, Respiratory Therapy Miami-Dade Community College Miami, Florida

1988 - 1990 Bachelors of Science, Nursing Florida International University Miami, Florida

$2002-2004 \quad$ Master of Science, Nursing University of Phoenix

Plantation, Florida

2007 - Present Doctoral Candidate, Florida International University

Miami, Florida

\section{PUBLICATIONS}

Birnbach DJ, Nevo I, Barnes S, Fitzpatrick M, Rosen LF, Everett-Thomas R, Sanko JS, Arheart KL. (2012) Do hospital visitors wash their hands? Assessing the use of alcoholbased hand sanitizer in a hospital lobby Am J of Infect Control 40:340-343

Nevo, I., Everett-Thomas, R., Fitzpatrick, M., Arheart, K., Mait, J., Birnbach, D.J. (2010) The efficacy of visual cues to improve HH compliance, Society of Simulation in Healthcare 5(5), 1-7

Lenchus, J., Kalidindi, V., Sanko, J., Everett-Thomas, R., Birnbach, D.J., (2010) Critical Elements to Advance Procedural Instruction: Knowledge, Attitude and Skills AAIM Insight

Lenchus, J. Issenberg, S.B. Murphy, D. Everett-Thomas, R. Erben, L. Kristopher Arheart, Birnbach, D.J. (2010) A blended approach to invasive bedside procedural instruction Medical Teacher

Lenchus, J., Murphy, D., Everett-Thomas, R. Erben, L., Arheart, K., Birnbach, D.J (2010) Implementing standardized procedural training at academic medical center: building core competencies and a better doctor JGME

Gluck P., Sanko, J., Everett-Thomas, R., Fitzpatrick, M., Lenchus, J., Birnbach, D.J (2010) Interns hand hygiene compliance is determined by factors (medical school, gender, specialty) 6(10) JGME

Ilya Shekhter, Nevo, I., Fitzpatrick, M., Everett-Thomas, R., Sanko, J., Birnbach, D.J (2009) Creating a Common Patient Safety Denominator: The Interns' Course JGME 12(09) 
Schulze A; Rieger-Fackeldey E; Gerhardt T; Claure N; Everett R; Bancalari E (2007)

Randomized crossover comparison of proportional assist ventilation and patient-

triggered ventilation in extremely low birth weight infants with evolving chronic lung disease Neonatology; Vol. 92 (1), 1-7. 